\title{
WELL-POSEDNESS AND REGULARITY FOR QUASILINEAR DEGENERATE PARABOLIC-HYPERBOLIC SPDE
}

\author{
By BENJAMIN GeSS ${ }^{*} \dagger$ AND MARTINA HofmanovÁ \\ Max Planck Institute for Mathematics in the Sciences*, Universität Bielefeld ${ }^{\dagger}$ and \\ Technical University Berlin
}

We study quasilinear degenerate parabolic-hyperbolic stochastic partial differential equations with general multiplicative noise within the framework of kinetic solutions. Our results are twofold: First, we establish new regularity results based on averaging techniques. Second, we prove the existence and uniqueness of solutions in a full $L^{1}$ setting requiring no growth assumptions on the nonlinearities. In addition, we prove a comparison result and an $L^{1}$ contraction property for the solutions, generalizing the results obtained in [Ann. Probab. 44 (2016) 1916-1955].

\section{CONTENTS}

1. Introduction . . . . . . . . . . . . . . . . . . . . . . . . 2496

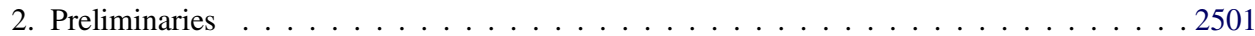

2.1. Notation . . . . . . . . . . . . . . . . . . . . . . . 2501

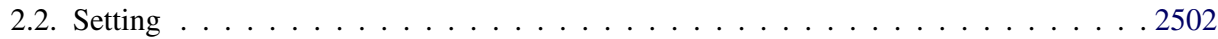

2.3. Kinetic solutions . . . . . . . . . . . . . . . . . . . . . 2504

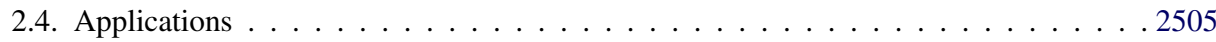

3. Regularity . . . . . . . . . . . . . . . . . . . . . . . 2507

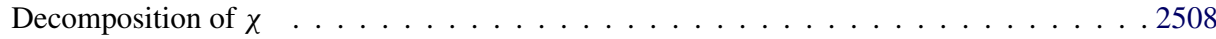

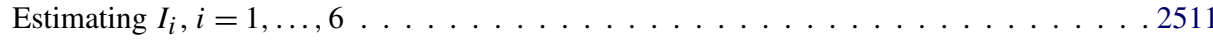

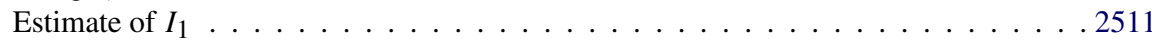

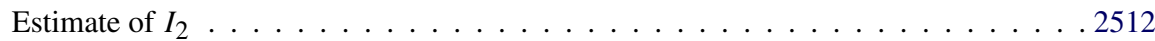

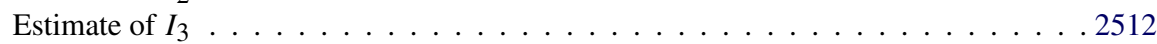

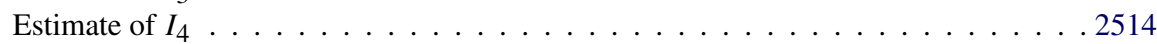

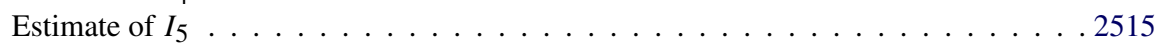

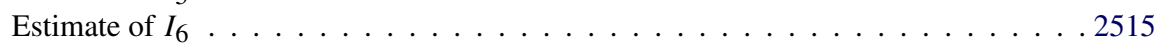

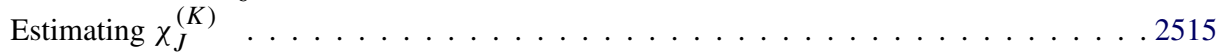

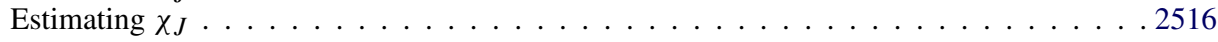

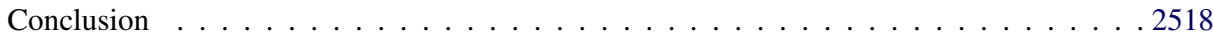

4. Well-posedness . . . . . . . . . . . . . . . . . . . . . . . . 2520

4.1. Uniqueness . . . . . . . . . . . . . . . . . . . . . 2520

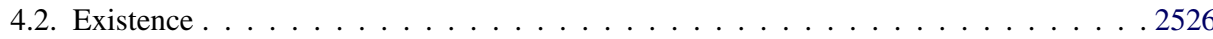

4.2.1. $L^{p}$-estimates . . . . . . . . . . . . . . . . . . . . . 2527

4.2.2. Decay of the kinetic measure . . . . . . . . . . . . . . . . 2528

Received June 2017.

MSC2010 subject classifications. 60H15, 35R60.

Key words and phrases. Quasilinear degenerate parabolic stochastic partial differential equation, kinetic formulation, kinetic solution, velocity averaging lemmas, renormalized solutions. 
4.2.3. The proof of existence . . . . . . . . . . . . . . . . . . . 2535 Appendix: Multiplier lemmas . . . . . . . . . . . . . . . . . . . . . . . . . . . 2541 References . . . . . . . . . . . . . . . . . . . . . . . . . . . 2542

1. Introduction. We study the regularity and well-posedness of quasilinear degenerate parabolic-hyperbolic SPDE of the form

$$
\begin{aligned}
\mathrm{d} u+\operatorname{div}(B(u)) \mathrm{d} t & =\operatorname{div}(A(u) \nabla u) \mathrm{d} t+\Phi(x, u) \mathrm{d} W, \\
u(0) & =u_{0},
\end{aligned}
$$

where $x \in \mathbb{T}^{N}, t \in(0, T), W$ is a cylindrical Wiener process, $u_{0} \in L^{1}\left(\mathbb{T}^{N}\right), B \in$ $C^{2}\left(\mathbb{R}, \mathbb{R}^{N}\right), A \in C^{1}\left(\mathbb{R}, \mathbb{R}^{N \times N}\right)$ takes values in the set of symmetric nonnegative definite matrices and $\Phi(x, u)$ are Lipschitz continuous diffusion coefficients.

Equations of this form arise in a wide range of applications including the convection-diffusion of an ideal fluid in porous media. The addition of a stochastic noise is often used to account for numerical, empirical or physical uncertainties. In view these applications, we aim to treat (1.1) under general assumptions on the coefficient $A, B$ and initial data $u_{0}$. In particular, the coefficients are not necessarily linear nor of linear growth and $A$ is not necessarily strictly elliptic. Hence, in particular, we include stochastic scalar conservation laws

$$
\mathrm{d} u+\operatorname{div}(B(u)) \mathrm{d} t=\Phi(x, u) \mathrm{d} W
$$

and stochastic porous media equations

$$
\mathrm{d} u+\operatorname{div}(B(u)) \mathrm{d} t=\Delta u^{[m]} \mathrm{d} t+\Phi(x, u) \mathrm{d} W,
$$

with $m>2$ and $u^{[m]}:=\operatorname{sgn}(u)|u|^{m}$.

One of the main points of this paper is to provide a full $L^{1}$ approach to (1.1). That is, we prove regularity estimates and well-posedness for (1.1) assuming no higher integrability. More precisely, only $u_{0} \in L^{1}\left(\mathbb{T}^{N}\right)$ and no growth assumptions on the nonlinearities $A, B$ are assumed, in contrast to the previous work [16]. In particular, no Lipschitz continuity (and thus linear growth) assumptions on $A, B$ are supposed.

This causes severe difficulties: First, the weak form of (1.1) is not necessarily well defined since $A(u), B(u)$ are not necessarily in $L_{\text {loc }}^{1}\left(\mathbb{T}^{N}\right)$ for $u \in L^{1}\left(\mathbb{T}^{N}\right)$. Therefore, renormalized solutions have to be considered (cf. [1, 9, 21]). Second, in order to prove the uniqueness of $L^{1}$ entropy solutions an equi-integrability condition or, equivalently, a decay condition for the entropy defect measure is required (see a more detailed discussion below). The usual decay condition used in the deterministic case is not applicable in the stochastic case and a new condition and proof has to be found. Third, in the stochastic case, the usual proof of existence of entropy solutions relying on the Crandall-Liggett theory of $m$-accretive operators in $L^{1}\left(\mathbb{T}^{N}\right)$ cannot be applied (cf. $\left.[8,12,13]\right)$. Instead, the construction of 
entropy solutions presented in this paper relies on new regularity estimates based on averaging techniques. The application of averaging techniques and the resulting regularity results are new for parabolic-hyperbolic SPDE of the type (1.1).

At the same time, $L^{1}\left(\mathbb{T}^{N}\right)$ is a natural space to consider the well-posedness for SPDE of the type (1.1) since the operators $\operatorname{div}(B(\cdot)), \operatorname{div}(A(\cdot) \nabla \cdot)$ are accretive in $L^{1}\left(\mathbb{T}^{N}\right)$ (cf. the discussion of the $e$-property after Theorem 1.1 below). In addition, and in contrast to the deterministic case, restricting to bounded solutions, and hence, by localization, to Lipschitz continuous coefficients $A, B$ in (1.1) does not seem to be sensible in the stochastic case, since in general no uniform $L^{\infty}$ bound will be satisfied by solutions to (1.1), due to the unboundedness of the driving noise $W$.

In the previous work [16], SPDE of the type (1.1) have been considered via a kinetic approach under more restrictive assumptions. More precisely, high moment bounds and integrability $u_{0} \in \bigcap_{p \geq 1} L^{p}\left(\Omega ; L^{p}\left(\mathbb{T}^{N}\right)\right)$, boundedness of the diffusion matrix $A$ and polynomial growth of $B^{\prime \prime}$ had to be assumed. This, in particular, rules out application to porous media equations. Due to these more restrictive assumptions all of the above mentioned difficulties do not appear in [16]. While we follow the principle setup to prove uniqueness of kinetic solutions, the proof has to be significantly extended in order to incorporate the necessity to work with renormalized solutions and the above mentioned weaker decay condition of the entropy defect measure. The essential difficulty in the proof of existence of solutions is the derivation of uniform estimates in some (fractional) Sobolev space. The respective arguments of [16] do not apply in the more general setting considered here. We therefore take a different route by adapting the (deterministic) averaging techniques by Tadmor and Tao [51] to the context of SPDE, which is entirely new.

As a particular example, (1.1) contains stochastic porous media equations

$$
\mathrm{d} u=\Delta u^{[m]} \mathrm{d} t+\Phi(x, u) \mathrm{d} W \quad \text { with } m>2 .
$$

Stochastic porous media equations have attracted a lot of interest in recent years (cf., e.g., [3, 47-49] and the references therein). All of these results rely on an $H^{-1}$ approach, that is, on treating $\Delta(\cdot)^{[\mathrm{m}]}$ as a monotone operator in $H^{-1}$. In contrast to the deterministic case, an $L^{1}$ approach to stochastic porous media equations had not yet been developed, since an analog of the concept of mild solutions in the Crandall-Liggett theory of $m$-accretive operators (cf. $[8,54]$ ) could not be found. However, the $L^{1}$ framework offers several advantages: First, more general classes of SPDE may be treated; second, contractive properties in $L^{1}$ norm are sometimes better than those in $H^{-1}$ norm. We next address these points in more detail.

Concerning the class of SPDE, informally speaking, the $H^{-1}$ approach relies on applying $(-\Delta)^{-1}$ to (1.2) which then allows to use the monotonicity of $\phi(u):=u^{[m]}$ in order to prove the uniqueness of solutions. While this works well for the operator $\Delta \phi(\cdot)$, the reader may easily check that this approach fails in the 
presence of hyperbolic terms $\operatorname{div} B(u)$ as in (1.1) and can only be applied to reaction diffusion equations

$$
\mathrm{d} u=\Delta u^{[m]} \mathrm{d} t+f(u) \mathrm{d} t+\Phi(x, u) \mathrm{d} W \quad \text { with } m>2
$$

under unnecessarily strong assumptions on the reaction term $f$ (cf., e.g., $[14,48]$ where (1.3) with $f$ satisfying rather restrictive assumptions has been considered). Roughly speaking, the problem is that the Nemytskii operator $u \mapsto f(u)$ is not necessarily monotone in $H^{-1}$ even if $f$ is a monotone function. This changes drastically in the $L^{1}$ setting, since both $u \mapsto \operatorname{div} B(u)$ and $u \mapsto f(u)$ are accretive operators on $L^{1}$ under relatively mild assumptions. In this paper, we resolve these issues by establishing a full $L^{1}$ approach to (1.1) based on entropy/kinetic methods. In particular, this extends available results on stochastic porous media equations by allowing hyperbolic terms $\operatorname{div} B(u)$ and our framework immediately ${ }^{1}$ extends to reaction terms $u \mapsto f(u)$ assuming only that $f$ is weakly monotone and $C^{2}$.

We proceed by stating the main well-posedness result obtained in this paper; see Theorem 4.3 and Theorem 4.9 below. The precise framework will be given in Section 2 below, and for specific examples, see Section 2.4 .

THEOREM 1.1. Let $u_{0} \in L^{1}\left(\mathbb{T}^{N}\right)$ and assume that $A^{\frac{1}{2}}$ is $\gamma$-Hölder continuous for some $\gamma>\frac{1}{2}$. Then kinetic solutions to (1.1) are unique. Moreover, if $u_{1}, u_{2}$ are kinetic solutions to (1.1) with initial data $u_{1,0}$ and $u_{2,0}$, respectively, then

$$
\underset{t \in[0, T]}{\operatorname{ess} \sup } \mathbb{E}\left\|\left(u_{1}(t)-u_{2}(t)\right)^{+}\right\|_{L^{1}\left(\mathbb{T}^{N}\right)} \leq\left\|\left(u_{1,0}-u_{2,0}\right)^{+}\right\|_{L^{1}\left(\mathbb{T}^{N}\right)} .
$$

Assume in addition that A, B satisfy a nondegeneracy assumption [cf. (2.3) below]. Then there exists a unique kinetic solution $u$ to (1.1) satisfying $u \in$ $C\left([0, T] ; L^{1}\left(\mathbb{T}^{N}\right)\right), \mathbb{P}$-a.s., and for all $p, q \in[1, \infty)$ there exists a constant $C>0$ such that

$$
\underset{t \in[0, T]}{\mathbb{E} \operatorname{ess} \sup }\|u(t)\|_{L^{p}}^{p q} \leq C\left(1+\left\|u_{0}\right\|_{L^{p}}^{p q}\right)
$$

The second direction of advantages of the $L^{1}$ approach lies in dynamical properties. A natural question for stochastic porous media equations is their long-time behavior, that is, the existence and uniqueness of invariant measures, mixing properties, etc. If $u, v$ are two solutions to (1.2) with initial conditions $u_{0}, v_{0}$ respectively, then

$$
\mathbb{E}\|u(t)-v(t)\|_{H^{-1}} \leq e^{C t}\left\|u_{0}-v_{0}\right\|_{H^{-1}} \quad \forall t \geq 0
$$

\footnotetext{
${ }^{1}$ We choose not to include the details on the treatment of reaction terms $f(u)$ in this paper, since their treatment is similar to the noise terms $\Phi(u) \mathrm{d} W$.
} 
for some constant $C>0$. The constant $C$ corresponds to the Lipschitz norm of $u \mapsto \Phi(u)$ as a map from $H^{-1}$ to $L_{2}\left(U ; H^{-1}\right)$. In particular, the dynamics induced by (1.2), in general, will not be nonexpanding in $H^{-1}$. In contrast, we show that

$$
\mathbb{E}\|u(t)-v(t)\|_{L^{1}} \leq\left\|u_{0}-v_{0}\right\|_{L^{1}} \quad \forall t \geq 0,
$$

that is, in the $L^{1}$ setting we can choose the constant $C$ in (1.4) to be zero. In particular, this implies the $e$-property (cf. [39]) for the associated Markovian semigroup $P_{t} f(x):=\mathbb{E} f\left(u_{t}^{x}\right)$ on $L^{1}\left(\mathbb{T}^{N}\right)$. The $e$-property has proven vital in the proof of existence and uniqueness of invariant measures for SPDE with degenerate noise (cf. [23, 29, 30, 39]).

For $x \in L^{1}\left(\mathbb{T}^{N}\right)$, let

$$
P_{x}:=\left(u^{x}\right)_{*} \mathbb{P},
$$

that is, $P_{x}$ is the law of $u^{x}$ on $C\left([0, \infty) ; L^{1}\left(\mathbb{T}^{N}\right)\right)$, where $u_{.}^{x}$ denotes the kinetic solution to (1.1) with initial condition $x$. We equip $C\left([0, \infty) ; L^{1}\left(\mathbb{T}^{N}\right)\right)$ with the canonical filtration $\mathcal{G}_{t}$ and evaluation maps $\pi_{t}(w):=w(t)$ for $w \in$ $C\left([0, \infty) ; L^{1}\left(\mathbb{T}^{N}\right)\right), t \geq 0$. As in [15], using Theorem 1.1, we obtain the following.

COROLlaRY 1.2. The family $\left\{P_{x}\right\}_{x \in L^{1}}$ is a time-homogeneous Markov process on $C\left([0, \infty) ; L^{1}\left(\mathbb{T}^{N}\right)\right)$ with respect to $\mathcal{G}_{t}$, that is,

$$
\mathbb{E}_{x}\left(F\left(\pi_{t+s}\right) \mid \mathcal{G}_{s}\right)=\mathbb{E}_{\pi_{s}}\left(F\left(\pi_{t}\right)\right) \quad P_{x} \text {-a.s. }
$$

In addition, $\left\{P_{x}\right\}_{x \in L^{1}\left(\mathbb{T}^{N}\right)}$ is Feller and satisfies the e-property (cf. [39]).

As mentioned above, we prove new regularity estimates for kinetic solutions to (1.1) of the type

$$
u(t) \in W^{\alpha, 1}\left(\mathbb{T}^{N}\right) \quad \text { for a.e. }(\omega, t),
$$

for some $\alpha>0$, based on stochastic velocity averaging lemmas. In particular, these estimates provide a smoothing property with respect to the initial data, which typically is the first step towards the construction of an invariant measure.

Even in the case of pure stochastic porous media equations (1.2) this extends previously available regularity results; for related deterministic results, see [7, 22, 37, 51], for stochastic hyperbolic conservation laws, see [17]. Our approach is mainly based on [51], but substantial difficulties due to the stochastic integral have to be overcome. Indeed, in most of the deterministic results (with the notable exception of [7]), the time variable does not play a special role and is regarded as another space variable and, in particular, space-time Fourier transforms are employed in the proofs. This changes in the stochastic case due to the irregularity of the noise in time. Therefore, it was argued in [17] that these methods are not suitable for the stochastic case and instead the approach of [7] which does not rely on Fourier transforms in time was employed. In the present paper, we rely on different arguments: We put forward averaging lemmas that rely on space-time Fourier 
transforms, Littlewood-Paley decomposition and a careful analysis of each of the appearing terms. As a consequence, we are able to estimate the stochastic integral as well as the kinetic measure term directly by averaging techniques, without any additional damping (as compared to [7, 17]). Moreover, our averaging lemmas apply to the case of nonhomogeneous equations, that is, PDEs with zero, first- and second-order terms and multiplicative noise.

More precisely, as a corollary of our main regularity result for (1.1), see Theorem 3.1 and Corollary 3.3 below. We obtain the following.

THEOREM 1.3. Assume that A, B satisfy a nondegeneracy condition [cf. (2.3) below] and are of polynomial growth of order $p$. Let $u$ be a kinetic solution to (1.1). Then

$$
\mathbb{E}\|u\|_{L^{1}\left([0, T] ; W^{s, 1}\left(\mathbb{T}^{N}\right)\right)} \lesssim\left\|u_{0}\right\|_{L_{x}^{2 p+3}}^{2 p+3}+1
$$

for some $s>0$.

We now proceed with a more detailed discussion of the comparison to the proof of well-posedness of entropy solutions for deterministic parabolic-hyperbolic PDE in the $L^{1}$ setting (cf. [11])

$$
\mathrm{d} u+\operatorname{div}(B(u)) \mathrm{d} t=\operatorname{div}(A(u) \nabla u) \mathrm{d} t .
$$

The inclusion of stochastic perturbation causes several additional difficulties. First, the proof of existence of solutions in [11] relies on the (simple) proof of $B V$ regularity of solutions to (1.5). Such a $B V$ estimate is not known in the stochastic case and does not seem to be easy to obtain (for a discussion of the necessity of such estimates in the construction of a solution, see Section 4.2 below). In the $L^{p}$ setting of [16], the $B V$ regularity was replaced by $W^{\sigma, 1}$ regularity which held true for smooth initial conditions based on similar calculations as in the uniqueness proof. Since we do not suppose any growth assumptions on the coefficients $A, B$ in (1.1), these arguments cannot be used here anymore. Therefore, we instead rely on regularity obtained by averaging techniques. In contrast to [16], our regularity estimates do not only prove preservation of some regularity of the initial condition, but yield a regularizing effect. Indeed, the initial condition does not need to be smooth for the averaging lemma to imply regularity for positive times. This fact in particular permits to reduce the number of approximation layers used in the proof of existence.

Second, the equi-integrability estimates encoded in the decay properties of the kinetic measure in the deterministic situation, that is, in the assumption (cf. [11], Definition 2.2(iv))

$$
\lim _{|\xi| \rightarrow \infty} \int m(t, x, \xi) \mathrm{d} t \mathrm{~d} x=0
$$


do not seem to be suitable in the stochastic case, since the multiplicative noise term $\Phi(x, u)$ is less well behaved in terms of these estimates (cf. Section 4.2.2 below). Indeed, the corresponding proof of a priori estimates proceeds along different lines than in the deterministic case (cf. Proposition 4.7 below). Therefore, we replace these decay estimates by the weaker decay condition

$$
\lim _{\ell \rightarrow \infty} \frac{1}{2^{\ell}} \mathbb{E} \int 1_{2^{\ell} \leq|\xi| \leq 2^{\ell+1}} m(t, x, \xi) \mathrm{d} t \mathrm{~d} x \mathrm{~d} \xi=0
$$

and prove the uniqueness of kinetic solutions under this weaker assumption. The above difficulty is due to working in an $L^{1}$-setting. In the situation of initial data/solutions in $L^{2}$, as in [16], the kinetic measure can be shown to have a.s. finite mass which allows to replace (1.6) by a stronger assumption.

The kinetic approach to (deterministic) scalar conservation laws was introduced by Lions, Perthame, Tadmor in [43] and extended to parabolic-hyperbolic PDE in [11], including PDE of porous media type. In the stochastic case, the well-posedness of such PDE had not previously been shown. Under more restrictive assumptions, as outlined above, the well-posedness to (1.1) has been obtained in [16]. Special cases of SPDE of the type (1.1) have attracted a lot of interest in recent years. For deterministic hyperbolic conservation laws, see $[6,36,40,42,43,45,46]$. Stochastic degenerate parabolic equations were studied in $[5,16,32]$ and stochastic conservation laws in [4, 10, 17-19, 24, 33, 35, 38, 50, 53]. Recently, also scalar conservation laws driven by rough paths have been considered in [20, 25, 34]. Other types of stochastic scalar conservation laws, for which randomness enters in form of a random flux have been considered in [26, 28, 41, 44]. Stochastic quasilinear parabolic-hyperbolic SPDE with random flux have been considered in [27].

The paper is organized as follows. In Section 2, we introduce the precise framework and the concept of kinetic solutions. Our main regularity result will be proven in Section 3. This is then used in Section 4 to prove the well-posedness for kinetic solutions.

\section{Preliminaries.}

2.1. Notation. In this paper, we use the brackets $\langle\cdot, \cdot\rangle$ to denote the duality between the space of distributions over $\mathbb{T}^{N} \times \mathbb{R}$ and $C_{c}^{\infty}\left(\mathbb{T}^{N} \times \mathbb{R}\right)$ and the duality between $L^{p}\left(\mathbb{T}^{N} \times \mathbb{R}\right)$ and $L^{q}\left(\mathbb{T}^{N} \times \mathbb{R}\right)$. If there is no danger of confusion, the same brackets will also denote the duality between $L^{p}\left(\mathbb{T}^{N}\right)$ and $L^{q}\left(\mathbb{T}^{N}\right)$. By $\mathcal{M}\left([0, T] \times \mathbb{T}^{N} \times \mathbb{R}\right)$, we denote the set of Radon measures on $[0, T] \times \mathbb{T}^{N} \times \mathbb{R}$ and $\mathcal{M}^{+}\left([0, T] \times \mathbb{T}^{N} \times \mathbb{R}\right)$ then contains nonnegative Radon measures and $\mathcal{M}_{b}\left([0, T] \times \mathbb{T}^{N} \times \mathbb{R}\right)$ contains finite measures. We also use the notation

$$
n(\phi)=\int_{[0, T] \times \mathbb{T}^{N} \times \mathbb{R}} \phi(t, x, \xi) \mathrm{d} n(t, x, \xi),
$$


where $n \in \mathcal{M}\left([0, T] \times \mathbb{T}^{N} \times \mathbb{R}\right), \phi \in C_{c}\left([0, T] \times \mathbb{T}^{N} \times \mathbb{R}\right)$. In order to signify that $n \in \mathcal{M}\left([0, T] \times \mathbb{T}^{N} \times \mathbb{R}\right)$ is only considered on $[0, T] \times \mathbb{T}^{N} \times D$ for some compact set $D \subset \mathbb{R}$ we write $n \mathbf{1}_{D}$. In particular,

$$
\left\|n \mathbf{1}_{D}\right\|_{\mathcal{M}_{t, x, \xi}}=\int_{[0, T] \times \mathbb{T}^{N} \times D} \mathrm{~d}|n|(t, x, \xi) .
$$

The differential operators of gradient $\nabla$, divergence div and Laplacian $\Delta$ are always understood with respect to the space variable $x$. For two matrices $A, B$ of the same size, we set $A: B:=\sum_{i j} a_{i j} b_{i j}$. Throughout the paper, we use the term representative for an element of a class of equivalence.

Finally, we use the letter $C$ to denote a generic constant that might change from one line to another. We also employ the notation $x \lesssim y$ if there exists a constant $C$ independent of the variables under consideration such that $x \leq C y$ and we write $x \sim y$ if $x \lesssim y$ and $y \lesssim x$. By $x \lesssim z y$, we mean that the corresponding proportional constant depends on the quantity $z$.

2.2. Setting. We now give the precise assumptions on each of the terms appearing in the above equation (1.1). We work on a finite-time interval $[0, T]$, $T>0$, and consider periodic boundary conditions: $x \in \mathbb{T}^{N}$ where $\mathbb{T}^{N}=$ $\mathbb{R}^{N} \mid\left(2 \pi \mathbb{Z}^{N}\right)$ is the $N$-dimensional torus. For the flux $B$, we assume

$$
B=\left(B_{1}, \ldots, B_{N}\right) \in C^{2}\left(\mathbb{R}, \mathbb{R}^{N}\right)
$$

and we set $b=\nabla B$. The diffusion matrix $A=\left(A_{i j}\right)_{i, j=1}^{N} \in C^{1}\left(\mathbb{R} ; \mathbb{R}^{N \times N}\right)$ is assumed to be symmetric, positive semidefinite and its square root $\sigma:=A^{\frac{1}{2}}$ is assumed to be locally $\gamma$-Hölder continuous for some $\gamma>1 / 2$, that is, for all $R>0$ there is a constant $C=C(R)$ such that

$$
|\sigma(\xi)-\sigma(\zeta)| \leq C(R)|\xi-\zeta|^{\gamma} \quad \forall \xi, \zeta \in \mathbb{R},|\xi|,|\zeta| \leq R
$$

We will further require a nondegeneracy condition for the symbol $\mathcal{L}$ associated to the kinetic form of (1.1)

$$
\mathcal{L}(i u, i n, \xi):=i(u+b(\xi) \cdot n)+n^{*} A(\xi) n .
$$

For $J, \delta>0$ and $\eta \in C_{b}^{\infty}(\mathbb{R})$ nonnegative, let

$$
\begin{aligned}
\Omega_{\mathcal{L}}^{\eta}(u, n ; \delta) & :=\{\xi \in \operatorname{supp} \eta ;|\mathcal{L}(i u, i n, \xi)| \leq \delta\}, \\
\omega_{\mathcal{L}}^{\eta}(J ; \delta) & :=\sup _{\substack{u \in \mathbb{R}, n \in \mathbb{Z}^{N} \\
|n| \sim J}}\left|\Omega_{\mathcal{L}}^{\eta}(u, n ; \delta)\right|
\end{aligned}
$$

and $\mathcal{L}_{\xi}:=\partial_{\xi} \mathcal{L}$. We suppose that there exist $\alpha \in(0,1), \beta>0$ and a measurable map $\vartheta \in L_{\text {loc }}^{\infty}(\mathbb{R} ;[1, \infty))$ such that

$$
\begin{gathered}
\omega_{\mathcal{L}}^{\eta}(J ; \delta) \lesssim \eta\left(\frac{\delta}{J^{\beta}}\right)^{\alpha} \\
\sup _{\substack{u \in \mathbb{R}, n \in \mathbb{Z}^{N} \\
|n| \sim J}} \sup _{\xi \in \operatorname{supp} \eta} \frac{\left|\mathcal{L}_{\xi}(i u, i n, \xi)\right|}{\vartheta(\xi)} \lesssim_{\eta} J^{\beta} \quad \forall \delta>0, J \gtrsim 1 .
\end{gathered}
$$


The requirement of a suitable nondegeneracy condition is classical in the theory of averaging lemmas and, therefore, it will be essential for Theorem 3.1. The localization $\eta$ and the weight $\vartheta$ give two possibilities to control the growth of $\mathcal{L}_{\xi}$ in $\xi$. In the proof of existence in Section 4.2.3, we employ (2.3) with $\vartheta \equiv 1$ and $\eta$ compactly supported which allows to obtain regularity of the localized average $\int_{\mathbb{R}} \chi_{u}(\xi) \eta(\xi) \mathrm{d} \xi$ without any further integrability assumptions on $u$. On the contrary, with a suitable choice of $\vartheta$, we may consider $\eta \equiv 1$ to obtain regularity of $u$ itself provided it possesses certain additional integrability. We refer the reader to Section 2.4 for further discussion of (2.3) as well as for application to particular examples.

Regarding the stochastic term, let $\left(\Omega, \mathscr{F},\left(\mathscr{F}_{t}\right)_{t \geq 0}, \mathbb{P}\right)$ be a stochastic basis with a complete, right-continuous filtration. Let $\mathcal{P}$ denote the predictable $\sigma$-algebra on $\Omega \times[0, T]$ associated to $\left(\mathscr{F}_{t}\right)_{t \geq 0}$. The initial datum $u_{0}$ is $\mathscr{F}_{0}$-measurable and the process $W$ is a cylindrical Wiener process, that is, $W(t)=\sum_{k \geq 1} \beta_{k}(t) e_{k}$ with $\left(\beta_{k}\right)_{k \geq 1}$ being mutually independent real-valued standard Wiener processes relative to $\left(\mathscr{F}_{t}\right)_{t \geq 0}$ and $\left(e_{k}\right)_{k \geq 1}$ a complete orthonormal system in a separable Hilbert space $\mathfrak{U}$. In this setting, we can assume without loss of generality that the $\sigma$ algebra $\mathscr{F}$ is countably generated and $\left(\mathscr{F}_{t}\right)_{t \geq 0}$ is the filtration generated by the Wiener process and the initial condition. For each $z \in L^{2}\left(\mathbb{T}^{N}\right)$, we consider a mapping $\Phi(z): \mathfrak{U} \rightarrow L^{2}\left(\mathbb{T}^{N}\right)$ defined by $\Phi(z) e_{k}=g_{k}(\cdot, z(\cdot))$. We suppose that $g_{k} \in C\left(\mathbb{T}^{N} \times \mathbb{R}\right)$ and there exists a sequence $\left(\alpha_{k}\right)_{k \geq 1}$ of positive numbers satisfying $D:=\sum_{k \geq 1} \alpha_{k}^{2}<\infty$ such that

$$
\left|g_{k}(x, 0)\right|+\left|\nabla_{x} g_{k}(x, \xi)\right|+\left|\partial_{\xi} g_{k}(x, \xi)\right| \leq \alpha_{k} \quad \forall x \in \mathbb{T}^{N}, \xi \in \mathbb{R} .
$$

Note that it follows from (2.4) that

$$
\left|g_{k}(x, \xi)\right| \leq \alpha_{k}(1+|\xi|) \quad \forall x \in \mathbb{T}^{N}, \xi \in \mathbb{R}
$$

and for all $x, y \in \mathbb{T}^{N}, \xi, \zeta \in \mathbb{R}$

$$
\sum_{k \geq 1}\left|g_{k}(x, \xi)-g_{k}(y, \zeta)\right|^{2} \leq C\left(|x-y|^{2}+|\xi-\zeta|^{2}\right)
$$

Consequently, denoting $G^{2}(x, \xi)=\sum_{k \geq 1}\left|g_{k}(x, \xi)\right|^{2}$ it holds

$$
G^{2}(x, \xi) \leq 2 D\left(1+|\xi|^{2}\right) \quad \forall x \in \mathbb{T}^{N}, \xi \in \mathbb{R}
$$

The conditions imposed on $\Phi$ imply that it maps $L^{2}\left(\mathbb{T}^{N}\right)$ to the space of HilbertSchmidt operators from $\mathfrak{U}$ to $L^{2}\left(\mathbb{T}^{N}\right)$, denoted by $L_{2}\left(\mathfrak{U} ; L^{2}\left(\mathbb{T}^{N}\right)\right)$. Thus, given a predictable process $u \in L^{2}\left(\Omega \times(0, T) ; L^{2}\left(\mathbb{T}^{N}\right)\right)$, the stochastic integral in (1.1) is a well-defined process taking values in $L^{2}\left(\mathbb{T}^{N}\right)$ (see [15] for a detailed construction). 
2.3. Kinetic solutions. Let us introduce the definition of kinetic solution as well as the related definitions used throughout this paper. It is a generalization of the concept of kinetic solution studied in [16], which is suited for establishing wellposedness in the $L^{1}$-framework, that is, for initial conditions in $L^{1}\left(\Omega ; L^{1}\left(\mathbb{T}^{N}\right)\right)$. In that case, the corresponding kinetic measure is not finite and one can only prove suitable decay at infinity.

Definition 2.1 (Kinetic measure). A mapping $m$ from $\Omega$ to $\mathcal{M}^{+}([0, T] \times$ $\left.\mathbb{T}^{N} \times \mathbb{R}\right)$, the set of nonnegative Radon measures over $[0, T] \times \mathbb{T}^{N} \times \mathbb{R}$, is said to be a kinetic measure provided:

(i) For all $\psi \in C_{c}\left([0, T) \times \mathbb{T}^{N} \times \mathbb{R}\right)$, the process

$$
\int_{[0, t] \times \mathbb{T}^{N} \times \mathbb{R}} \psi(s, x, \xi) \mathrm{d} m(s, x, \xi)
$$

is predictable.

(ii) Decay of $m$ for large $\xi$ : it holds true that

$$
\lim _{\ell \rightarrow \infty} \frac{1}{2^{\ell}} \mathbb{E} m\left(A_{2^{\ell}}\right)=0
$$

where

$$
A_{2^{\ell}}=[0, T] \times \mathbb{T}^{N} \times\left\{\xi \in \mathbb{R} ; 2^{\ell} \leq|\xi| \leq 2^{\ell+1}\right\}
$$

Definition 2.2 (Kinetic solution). A function $u \in L^{1}(\Omega \times[0, T], \mathcal{P}, \mathrm{d} \mathbb{P} \otimes$ $\left.\mathrm{d} t ; L^{1}\left(\mathbb{T}^{N}\right)\right)$ is called a kinetic solution to (1.1) with initial datum $u_{0}$ if the following conditions are satisfied:

(i) For all $\phi \in C_{c}^{\infty}(\mathbb{R}), \phi \geq 0$,

$$
\operatorname{div} \int_{0}^{u} \phi(\zeta) \sigma(\zeta) \mathrm{d} \zeta \in L^{2}\left(\Omega \times[0, T] \times \mathbb{T}^{N}\right) .
$$

(ii) For all $\phi_{1}, \phi_{2} \in C_{c}^{\infty}(\mathbb{R}), \phi_{1}, \phi_{2} \geq 0$, the following chain rule formula holds true in $L^{2}\left(\Omega \times[0, T] \times \mathbb{T}^{N}\right)$

$$
\operatorname{div} \int_{0}^{u} \phi_{1}(\zeta) \phi_{2}(\zeta) \sigma(\zeta) \mathrm{d} \zeta=\phi_{1}(u) \operatorname{div} \int_{0}^{u} \phi_{2}(\zeta) \sigma(\zeta) \mathrm{d} \zeta
$$

(iii) Let $\phi \in C_{c}^{\infty}(\mathbb{R}), \phi \geq 0$, and let $n^{\phi}: \Omega \rightarrow \mathcal{M}^{+}\left([0, T] \times \mathbb{T}^{N}\right)$ be defined as follows: for all $\varphi \in C_{c}^{\infty}\left([0, T] \times \mathbb{T}^{N}\right)$,

$$
n^{\phi}(\varphi)=\int_{0}^{T} \int_{\mathbb{T}^{N}} \varphi(t, x)\left|\operatorname{div} \int_{0}^{u} \sqrt{\phi(\zeta)} \sigma(\zeta) \mathrm{d} \zeta\right|^{2} \mathrm{~d} x \mathrm{~d} t .
$$

There exists a kinetic measure $m$ such that, for all $\varphi \in C_{c}^{\infty}\left([0, T] \times \mathbb{T}^{N}\right), \varphi \geq 0$ and $\phi \in C_{c}^{\infty}(\mathbb{R}), \phi \geq 0$, it holds $m(\varphi \phi) \geq n^{\phi}(\varphi), \mathbb{P}$-a.s., and, in addition, the pair 
$\left(f=\mathbf{1}_{u>\xi}, m\right)^{2}$ satisfies, for all $\varphi \in C_{c}^{\infty}\left([0, T) \times \mathbb{T}^{N} \times \mathbb{R}\right), \mathbb{P}$-a.s.

$$
\begin{aligned}
\int_{0}^{T}\left\langle f(t), \partial_{t} \varphi(t)\right\rangle \mathrm{d} t+\left\langle f_{0}, \varphi(0)\right\rangle+\int_{0}^{T}\langle f(t), b \cdot \nabla \varphi(t)\rangle \mathrm{d} t \\
\quad+\int_{0}^{T}\left\langle f(t), A: \mathrm{D}^{2} \varphi(t)\right\rangle \mathrm{d} t \\
=-\sum_{k \geq 1} \int_{0}^{T} \int_{\mathbb{T}^{N}} g_{k}(x, u(t, x)) \varphi(t, x, u(t, x)) \mathrm{d} x \mathrm{~d} \beta_{k}(t) \\
\quad-\frac{1}{2} \int_{0}^{T} \int_{\mathbb{T}^{N}} G^{2}(x, u(t, x)) \partial_{\xi} \varphi(t, x, u(t, x)) \mathrm{d} x \mathrm{~d} t+m\left(\partial_{\xi} \varphi\right) .
\end{aligned}
$$

The definition of a kinetic solution given in Definition 2.2 generalizes the definition of kinetic solutions given in [16], Definition 2.2, which applies to the case of high integrability, that is, for $u \in L^{p}\left(\Omega ; L^{p}\left([0, T] \times \mathbb{T}^{N}\right)\right)$ for all $p \geq 1$. We note that in particular the chain rule (2.7) is weaker than the corresponding version [[16], (2.5)]. As a consequence, the parabolic dissipation $n^{\phi}$ does not necessarily define a measure with respect to the variable $\xi$. It was already mentioned above that the kinetic measure $m$ is generally not a finite measure and the decay assumption from Definition 2.1(ii), is weaker than the one in [16], Definition 2.1(ii).

REMARK 2.3. Let $u \in L^{p}\left(\Omega ; L^{p}\left([0, T] \times \mathbb{T}^{N}\right)\right)$ for all $p \geq 1$. Then $u$ is a kinetic solution to (1.1) in the sense of [16], Definition 2.2, if and only if $u$ is a kinetic solution in the sense of Definition 2.2.

REMARK 2.4. We emphasize that a kinetic solution is, in fact, a class of equivalence in $L^{1}\left(\Omega \times[0, T] ; L^{1}\left(\mathbb{T}^{N}\right)\right)$ so not necessarily a stochastic process in the usual sense. The term representative is then used to denote an element of this class of equivalence.

We will further use the notions of Young measures and kinetic functions for the definition of which we refer to [16], Definition 2.4, Definition 2.5, Remark 2.6.

2.4. Applications. In this section, we consider the model example of a convection-diffusion SPDE with polynomial nonlinearities, that is, let $N=1$ and consider

$$
\mathrm{d} u+\partial_{x}\left(\frac{u^{k}}{k}\right) \mathrm{d} t=\partial_{x}\left(|u|^{m-1} \partial_{x} u\right) \mathrm{d} t+\Phi(x, u) \mathrm{d} W
$$

\footnotetext{
${ }^{2}$ Here, $\mathbf{1}_{u>\xi}$ is considered as a function of four variables, namely $(\omega, t, x, \xi) \mapsto \mathbf{1}_{u(\omega, t, x)>\xi}$.
} 
that is, (1.1) with $b(\xi)=B^{\prime}(\xi)=\xi^{k-1}, A(\xi)=|\xi|^{m-1}$, for $k \geq 2, m>2$. Hence,

$$
\begin{aligned}
\mathcal{L}(i u, i n, \xi) & =i\left(u+\xi^{k-1} n\right)+|\xi|^{m-1} n^{2}, \\
\left|\mathcal{L}_{\xi}(i u, i n, \xi)\right| & \lesssim|\xi|^{k-2}|n|+|\xi|^{m-2} n^{2} .
\end{aligned}
$$

For $\eta \in C_{b}^{\infty}(\mathbb{R})$ and $u \in \mathbb{R}, n \in \mathbb{Z},|n| \sim J$, we consider

$$
\Omega_{\mathcal{L}}^{\eta}(u, n ; \delta)=\left\{\xi \in \operatorname{supp} \eta ;\left.\left|i\left(u+\xi^{k-1} n\right)+\right| \xi\right|^{m-1} n^{2} \mid \leq \delta\right\}
$$

and observe that $\Omega_{\mathcal{L}}^{\eta}(u, n ; \delta) \subset \Omega_{A} \cap \Omega_{b}$ where

$$
\begin{aligned}
\Omega_{A} & :=\left\{\xi \in \operatorname{supp} \eta ;|\xi|^{m-1}|n|^{2} \leq \delta\right\}, \\
\Omega_{b} & :=\left\{\xi \in \operatorname{supp} \eta ;\left|i\left(u+\xi^{k-1} n\right)\right| \leq \delta\right\} .
\end{aligned}
$$

Note that the set $\Omega_{A}$ is localized around 0 in the sense that

$$
\Omega_{A}=\left\{\xi \in \operatorname{supp} \eta ;|\xi| \leq\left(\frac{\delta}{J^{2}}\right)^{\frac{1}{m-1}}\right\},
$$

whereas the set $\Omega_{b}$ is moving according to the value of $u$ :

$$
\Omega_{b}=\left\{\xi \in \operatorname{supp} \eta ;\left(\frac{u-\delta}{J}\right)^{\frac{1}{k-1}} \leq \xi \leq\left(\frac{u+\delta}{J}\right)^{\frac{1}{k-1}}\right\} .
$$

In view of the second part of the condition (2.3), we choose $\beta=2$ whenever a second-order operator is present. Therefore, we set $\beta=2$ and $\alpha=\frac{1}{m-1}$, which yields the first part of (2.3) independently of $\eta$

$$
\omega_{\mathcal{L}}^{\eta}(J ; \delta) \lesssim\left(\frac{\delta}{J^{2}}\right)^{\frac{1}{m-1}} .
$$

Regarding the second condition, it is necessary to control the $\xi$-growth in (2.10). Our formulation of the nondegeneracy condition (2.3) offers two ways of doing so: either using a (compactly supported) localization $\eta$ or a weight $\vartheta$. Using the first approach, Theorem 3.1 yields regularity of the localized average $\bar{\eta}(u)=\int_{\mathbb{R}} \chi_{u(t, x)}(\xi) \eta(\xi) \mathrm{d} \xi$ without any further integrability assumptions on the solution $u$. On the other hand, the second approach allows to obtain regularity of the solution $u$ itself, that is, setting $\eta \equiv 1$, but requires higher integrability of $u$. To be more precise, in the case of (2.10) we set $\vartheta(\xi)=1+|\xi|^{k \vee m-2}$ and assume that $u \in L^{p}\left(\Omega \times[0, T] \times \mathbb{T}^{N}\right)$ for $p=2(k \vee m-2)+3$.

In the case of a purely hyperbolic equation with a polynomial nonlinearity $b(\xi)=\xi^{k-1}, k \geq 2$, we obtain

$$
\Omega_{\mathcal{L}}^{\eta}(u, n ; \delta)=\left\{\xi \in \operatorname{supp} \eta ; \frac{u-\delta}{J} \leq \xi^{k-1} \leq \frac{u+\delta}{J}\right\},
$$

which implies the first condition in (2.3) independently of $\eta$ with $\alpha=\frac{1}{k-1}, \beta=1$. For the second condition, we proceed the same way as above. 
3. Regularity. In this section, we establish a regularity result for solutions to (1.1), based on averaging techniques. Throughout this section, we use the following notation: for a kinetic solution $u$, let $\chi:=\chi_{u}=\mathbf{1}_{u>\xi}-\mathbf{1}_{0>\xi}$. Then we have, in the sense of distributions,

$$
\partial_{t} \chi+b(\xi) \cdot \nabla \chi-A(\xi): D^{2} \chi=\partial_{\xi} q-\sum_{k=1}^{\infty}\left(\partial_{\xi} \chi\right) g_{k} \dot{\beta}_{k}+\sum_{k=1}^{\infty} \delta_{0} g_{k} \dot{\beta_{k}},
$$

where $q=m-\frac{1}{2} G^{2} \delta_{u=\xi}$. For $\eta \in C_{b}^{\infty}(\mathbb{R})$, let $\bar{\eta} \in C^{\infty}$ be such that $\bar{\eta}^{\prime}=\eta$ and $\bar{\eta}(0)=0$. We then have

$$
\bar{\eta}(u)=\int_{\mathbb{R}} \chi_{u(t, x)}(\xi) \eta(\xi) \mathrm{d} \xi .
$$

THEOREM 3.1. Assume (2.1), (2.4). Let $\eta \in C_{b}^{\infty}\left(\mathbb{R} ; \mathbb{R}_{+}\right)$and assume that there are $\alpha \in(0,1), \beta>0$ and a measurable map $\vartheta \in L_{\mathrm{loc}}^{\infty}(\mathbb{R} ;[1, \infty))$ such that (2.3) is satisfied. Let $\Theta_{\eta}: \mathbb{R} \rightarrow \mathbb{R}_{+}$such that $\Theta_{\eta}^{\prime}=\left(|\xi|^{2}+1\right) \vartheta^{2}(\xi)\left(\eta(\xi)+\left|\eta^{\prime}\right|(\xi)\right)$. If $u$ is a kinetic solution to (1.1), then

$$
\bar{\eta}(u)=\int_{\mathbb{R}} \chi_{u(t, x)}(\xi) \eta(\xi) \mathrm{d} \xi \in L^{r}\left(\Omega \times[0, T] ; W^{s, r}\left(\mathbb{T}^{N}\right)\right), \quad s<\frac{\alpha^{2} \beta}{6(1+2 \alpha)}
$$

with $\frac{1}{r}>\frac{1-\theta}{2}+\frac{\theta}{1}, \theta=\frac{\alpha}{4+\alpha}$ and

$$
\begin{aligned}
& \|\bar{\eta}(u)\|_{L^{r}\left(\Omega \times[0, T] ; W^{s, r}\left(\mathbb{T}^{N}\right)\right)} \\
& \quad \lesssim_{\eta}\left\|\bar{\eta}\left(\left|u_{0}\right|\right)\right\|_{L_{\omega, x}^{1}}^{1 / 2}+\left\|\Theta_{\eta}(|u|)\right\|_{L_{\omega, t, x}^{1}}^{1 / 2} \\
& \quad+\sup _{0 \leq t \leq T}\|\bar{\eta}(|u|)\|_{L_{\omega, x}^{1}}+\left\|m \vartheta\left(\eta+\left|\eta^{\prime}\right|\right)\right\|_{L_{\omega}^{1} \mathcal{M}_{t, x, \xi}}+1,
\end{aligned}
$$

where the constant in the inequality depends on $b$ and $A$ via the constants appearing in (2.3) only and on $\eta$ only via its $C^{1}$ norm.

REMARK 3.2. If $\eta$ is compactly supported, then we may always take $\vartheta \equiv 1$ in (2.3). Furthermore, in this case the right-hand side in (3.2) is always finite.

In order to deduce regularity for $u$ itself, we choose $\eta \equiv 1$. If $\vartheta$ is a polynomial of order $p$, then $\Theta_{\eta}$ is a polynomial of order $2 p+3$ and by Lemma 4.6 below we have $\left\|\Theta_{\eta}(|u|)\right\|_{L_{\omega, t, x}^{1}}^{\frac{1}{2}} \lesssim\left\|u_{0}\right\|_{L_{\omega, t, x}^{2 p+3}}^{\frac{2 p+3}{2}}+1$ and $\|m \vartheta\|_{L_{\omega}^{1} \mathcal{M}_{t, x, \xi}} \lesssim\left\|u_{0}\right\|_{L^{p+2}}^{p+2}+1$. In conclusion, we obtain the following.

COROLlary 3.3. Suppose (2.3) is satisfied for $\eta \equiv 1$ and $\vartheta$ being a polynomial of order $p$. Let $u$ be the kinetic solution ${ }^{3}$ to (1.1). Then

$$
\|u\|_{L^{r}\left(\Omega \times[0, T] ; W^{s, r}\left(\mathbb{T}^{N}\right)\right)} \lesssim\left\|u_{0}\right\|_{L_{\omega, x}^{2 p+3}}^{2 p+3}+1 .
$$

\footnotetext{
${ }^{3}$ Well-posedness of kinetic solutions to (1.1) is proved in Section 4 below.
} 
ProOF OF THEOREM 3.1. The proof proceeds in several steps. In the first step, the solution $\chi=\chi_{u}=f-\mathbf{1}_{0>\xi}$ is decomposed into Littlewood-Paley blocks $\chi_{J}$ and subsequently each Littlewood-Paley block is decomposed according to the degeneracy of the symbol $\mathcal{L}(i u, i n, \xi)$. This decomposition of $f$ serves as the basis of the following averaging techniques. In the second step, each part of the decomposition is estimated separately, relying on the nondegeneracy condition (2.3). In the last step, these estimates are combined and interpolated in order to deduce the regularity of $f$.

The principle idea of the above decomposition of $f$ follows [51]. However, the stochastic integral in (1.1) leads to additional difficulties and requires a different treatment of the time variable. This is resolved here by passing to the mild form [cf. (3.4) below] and then estimating all occurring terms separately, interpolating the estimates in the end.

Decomposition of $\chi$. We introduce a cut-off in time, that is, let $\phi=\phi^{\lambda} \in$ $C^{1}([0, \infty))$ such that $0 \leq \phi \leq 1, \phi \equiv 1$ on $[0, T-\lambda], \phi \equiv 0$ on $[T, \infty)$ and $\left|\partial_{t} \phi\right| \leq \frac{1}{\lambda}$ for some $\lambda \in(0,1)$ to be eventually sent to 0 . For notational simplicity, we omit the superscript $\lambda$ in the following computations and let it only reappear at the end of the proof, where the passage to the limit in $\lambda$ is discussed.

Then, $\chi \phi$ solves, in the sense of distributions,

$$
\begin{aligned}
\partial_{t}(\chi \phi) & +b(\xi) \cdot \nabla(\chi \phi)-A(\xi): D^{2}(\chi \phi) \\
= & \partial_{\xi}(\phi q)-\sum_{k=1}^{\infty} \partial_{\xi}(\chi \phi) g_{k} \dot{\beta_{k}}+\sum_{k=1}^{\infty} \delta_{0} \phi g_{k} \dot{\beta_{k}}+\chi \partial_{t} \phi .
\end{aligned}
$$

Next, we decompose $\chi$ into Littlewood-Paley blocks $\chi_{J}$, such that the Fourier transform in space $\widehat{\chi J}$ is supported by frequencies $|n| \sim J$ for $J$ dyadic. This is achieved by taking a smooth partition of unity $1 \equiv \varphi_{0}(z)+\sum_{J \gtrsim 1} \varphi\left(J^{-1} z\right)$ such that $\varphi_{0}$ is a bump function supported inside the ball $|z| \leq 2$ and $\varphi$ is a bump function supported in the annulus $\frac{1}{2} \leq|z| \leq 2$, and setting

$$
\begin{aligned}
\chi_{0}(t, x, \xi) & :=\mathcal{F}_{x}^{-1}\left[\varphi_{0}(n) \hat{\chi}(t, n, \xi)\right](x), \\
\chi_{J}(t, x, \xi) & :=\mathcal{F}_{x}^{-1}\left[\varphi\left(\frac{n}{J}\right) \hat{\chi}(t, n, \xi)\right](x), \quad J \gtrsim 1 .
\end{aligned}
$$

This leads to the decomposition $\chi=\chi_{0}+\sum_{J \gtrsim_{1}} \chi_{J}$. The regularity of $\chi_{0}$ being trivial, we only focus on the estimate of $\chi_{J}$ for $J \gtrsim 1$. Localizing (3.3) in Littlewood-Paley blocks yields

$$
\begin{aligned}
\partial_{t}\left(\chi_{J} \phi\right) & +b(\xi) \cdot \nabla\left(\chi_{J} \phi\right)-A(\xi): D^{2}\left(\chi_{J} \phi\right) \\
= & \partial_{\xi}\left(\phi q_{J}\right)-\sum_{k=1}^{\infty} \partial_{\xi}\left(\chi \phi g_{k}\right)_{J} \dot{\beta}_{k}+\sum_{k=1}^{\infty}\left(\chi \phi \partial_{\xi} g_{k}\right)_{J} \dot{\beta}_{k} \\
& +\sum_{k=1}^{\infty} \delta_{0} \phi\left(g_{k}\right)_{J} \dot{\beta}_{k}+\chi_{J} \partial_{t} \phi .
\end{aligned}
$$


After a preliminary step of regularization, we may test by $S^{*}(T-t) \varphi$ for $\varphi \in$ $C\left(\mathbb{T}^{N}\right)$ in (3.1), where $S(t)$ denotes the solution semigroup to the linear operator $\chi \mapsto b(\xi) \cdot \nabla \chi-A(\xi): D^{2} \chi$. This leads to the mild form

$$
\begin{aligned}
\left(\chi_{J} \phi\right)(t)= & S(t) \chi_{J}(0)+\int_{0}^{t} S(t-s) \partial_{\xi}\left(\phi q_{J}\right) \mathrm{d} s \\
& -\sum_{k=1}^{\infty} \int_{0}^{t} S(t-s) \partial_{\xi}\left(g_{k} \chi \phi\right)_{J} \mathrm{~d} \beta_{k}(s) \\
& +\sum_{k=1}^{\infty} \int_{0}^{t} S(t-s)\left(\left(\partial_{\xi} g_{k}\right) \chi \phi\right)_{J} \mathrm{~d} \beta_{k}(s) \\
& +\sum_{k=1}^{\infty} \int_{0}^{t} S(t-s) \delta_{0} \phi g_{k, J} \mathrm{~d} \beta_{k}(s)+\int_{0}^{t} S(t-s) \chi_{J} \partial_{t} \phi \mathrm{d} s
\end{aligned}
$$

where we have used

$$
\begin{aligned}
\varphi\left(\frac{n}{J}\right) & \mathcal{F}_{x}\left[\int_{0}^{t} S(t-s) \partial_{\xi}(\chi \phi) g_{k} \mathrm{~d} \beta_{k}(s)\right](n) \\
= & \int_{0}^{t} \mathrm{e}^{-\left(i b(\xi) \cdot n+n^{*} A(\xi) n\right)(t-s)} \partial_{\xi} \mathcal{F}_{x}\left(g_{k} \chi \phi\right)_{J}(s, n, \xi) \mathrm{d} \beta_{k}(s) \\
& -\int_{0}^{t} \mathrm{e}^{-\left(i b(\xi) \cdot n+n^{*} A(\xi) n\right)(t-s)} \mathcal{F}_{x}\left(\left(\partial_{\xi} g_{k}\right) \chi \phi\right)_{J}(s, n, \xi) \mathrm{d} \beta_{k}(s)
\end{aligned}
$$

and

$$
\begin{aligned}
\varphi\left(\frac{n}{J}\right) & \mathcal{F}_{x}\left[\int_{0}^{t} S(t-s) \delta_{0} \phi g_{k} \mathrm{~d} \beta_{k}(s)\right](n) \\
& =\int_{0}^{t} \mathrm{e}^{-\left(i b(\xi) \cdot n+n^{*} A(\xi) n\right)(t-s)} \delta_{0} \phi \widehat{g_{k, J}}(n, \xi) \mathrm{d} \beta_{k}(s) .
\end{aligned}
$$

For $J \gtrsim 1$ fixed, we next decompose the action in $\xi$-variable according to the degeneracy of the operator $\mathcal{L}(i u, i n, \xi)$. Namely, for $K$ dyadic, let $1 \equiv \psi_{0}(z)+$ $\sum_{K \gtrsim 1} \psi_{1}\left(K^{-1} z\right)$ be a smooth partition of unity such that $\psi_{0}$ is a bump function supported inside the ball $|z| \leq 2$ and $\psi_{1}$ is a bump function supported in the annulus $\frac{1}{2} \leq|z| \leq 2$, and write

$$
\begin{aligned}
\mathbf{1}_{0 \leq t}\left(\chi_{J} \phi\right)(t, x, \xi) \\
=\mathcal{F}_{t x}^{-1}\left[\psi_{0}\left(\frac{\mathcal{L}(i u, i n, \xi)}{\delta}\right) \mathcal{F}_{t x}\left[\mathbf{1}_{0 \leq t}\left(\chi_{J} \phi\right)\right](u, n, \xi)\right](t, x) \\
\quad+\sum_{K \gtrsim 1} \mathcal{F}_{t x}^{-1}\left[\psi_{1}\left(\frac{\mathcal{L}(i u, i n, \xi)}{\delta K}\right) \mathcal{F}_{t x}\left[\mathbf{1}_{0 \leq t}\left(\chi_{J} \phi\right)\right](u, n, \xi)\right](t, x) \\
=: \chi_{J}^{(0)}(t, x, \xi)+\sum_{K \gtrsim 1} \chi_{J}^{(K)}(t, x, \xi) .
\end{aligned}
$$


Hence, we consider the decomposition

$$
\mathbf{1}_{0 \leq t} \chi \phi=\mathbf{1}_{0 \leq t} \chi_{0} \phi+\sum_{J \gtrsim 1}\left(\chi_{J}^{(0)}(t, x, \xi)+\sum_{K \gtrsim 1} \chi_{J}^{(K)}(t, x, \xi)\right) .
$$

Since $\psi_{0}$ is supported at the degeneracy, we will apply a trivial estimate. However, $\psi_{1}$ is supported away from the degeneracy and, therefore, we may use the equation and the nondegeneracy assumption (2.3). From (3.4), we obtain

$$
\begin{aligned}
\chi_{J}^{(K)}(t, x, \xi)= & \mathcal{F}_{t x}^{-1} \psi_{1}\left(\frac{\mathcal{L}(i u, i n, \xi)}{\delta K}\right) \mathcal{F}_{t x}\left[\mathbf{1}_{0 \leq t} S(t) \chi_{0, J}\right. \\
& +\mathbf{1}_{0 \leq t} \int_{0}^{t} S(t-s) \partial_{\xi}\left(\phi q_{J}\right) \mathrm{d} s \\
& -\mathbf{1}_{0 \leq t} \sum_{k=1}^{\infty} \int_{0}^{t} S(t-s) \partial_{\xi}\left(g_{k} \chi \phi\right)_{J} \mathrm{~d} \beta_{k}(s) \\
& +\mathbf{1}_{0 \leq t} \sum_{k=1}^{\infty} \int_{0}^{t} S(t-s)\left(\left(\partial_{\xi} g_{k}\right) \chi \phi\right)_{J} \mathrm{~d} \beta_{k}(s) \\
& +\mathbf{1}_{0 \leq t} \sum_{k=1}^{\infty} \int_{0}^{t} S(t-s) \delta_{0} \phi g_{k, J} \mathrm{~d} \beta_{k}(s) \\
& \left.+\mathbf{1}_{0 \leq t} \int_{0}^{t} S(t-s) \chi_{J} \partial_{t} \phi \mathrm{d} s\right](t, x) .
\end{aligned}
$$

Multiplying the above by $\eta \in C_{b}^{\infty}(\mathbb{R})$ and integrating over $\xi \in \mathbb{R}$, we set

$$
\int_{\mathbb{R}} \chi_{J}^{(K)}(t, x, \xi) \eta(\xi) \mathrm{d} \xi=: I_{1}+I_{2}-I_{3}+I_{4}+I_{5}+I_{6}
$$

and we estimate the right-hand side term by term below. Note that since

$$
\begin{aligned}
& \mathcal{F}_{t x}\left[\mathbf{1}_{0 \leq t} \int_{0}^{t} S(t-s) \partial_{\xi}\left(g_{k} \chi \phi\right)_{J} \mathrm{~d} \beta_{k}(s)\right] \\
& \left.=\mathcal{F}_{t}\left[\mathbf{1}_{0 \leq t} \int_{0}^{t} \mathrm{e}^{-\left(i b(\xi) \cdot n+n^{*} A(\xi) n\right)(t-s)} \partial_{\xi} \widehat{\left(g_{k} \chi \phi\right.}\right)_{J}(s, \xi, n) \mathrm{d} \beta_{k}(s)\right] \\
& \left.=\frac{1}{(2 \pi)^{1 / 2}} \iint \mathbf{1}_{0 \leq s \leq t} \mathrm{e}^{-\left(i b(\xi) \cdot n+n^{*} A(\xi) n\right)(t-s)} \partial_{\xi} \widehat{\left(g_{k} \chi \phi\right)}\right)_{J} \mathrm{~d} \beta_{k}(s) \mathrm{e}^{-i t u} \mathrm{~d} t \\
& =\int_{0}^{\infty} \mathrm{e}^{-\left(i b(\xi) \cdot n+n^{*} A(\xi) n\right) r} \mathrm{e}^{-i r u} \mathrm{~d} r \\
& \times \frac{1}{(2 \pi)^{1 / 2}} \int_{0}^{\infty} \partial_{\xi}\left(\widehat{g_{k} \chi \phi}\right)_{J}(s, \xi, n) \mathrm{e}^{-i s u} \mathrm{~d} \beta_{k}(s) \\
& =\frac{1}{i(u+b(\xi) \cdot n)+n^{*} A(\xi) n} \frac{1}{(2 \pi)^{1 / 2}} \int_{0}^{\infty} \partial_{\xi}\left(\widehat{\left(g_{k} \chi \phi\right.}\right)_{J}(s, \xi, n) \mathrm{e}^{-i s u} \mathrm{~d} \beta_{k}(s),
\end{aligned}
$$


we have

$$
\begin{aligned}
& I_{3}=\frac{1}{(2 \pi)^{1 / 2}} \frac{1}{(\delta K)} \\
& \times \int_{\mathbb{R}} \mathcal{F}_{t x}^{-1}\left[\tilde{\psi} \sum_{k=1}^{\infty} \int_{0}^{\infty} \mathrm{e}^{-i s u} \partial_{\xi}\left(\widehat{g_{k} \chi \phi}\right)_{J}(s, \xi, n) \mathrm{d} \beta_{k}(s)\right] \eta(\xi) \mathrm{d} \xi,
\end{aligned}
$$

where we set $\tilde{\psi}(z):=\psi_{1}(z) / z$ and for notational simplicity omitted the argument, that is,

$$
\tilde{\psi}=\tilde{\psi}\left(\frac{\mathcal{L}(i u, i n, \xi)}{\delta K}\right)
$$

in $I_{3}$. The same convention will often be used in the sequel. We argue similarly for the remaining terms $I_{i}$, for example, for $I_{2}$ we note that

$$
\begin{aligned}
\mathcal{F}_{t x}\left[\mathbf{1}_{0 \leq t} \int_{0}^{t} S(t-s) \partial_{\xi}\left(\phi q_{J}\right) \mathrm{d} s\right] \\
\quad=\frac{1}{i(u+b(\xi) \cdot n)+n^{*} A(\xi) n} \mathcal{F}_{t x}\left[\mathbf{1}_{0 \leq t} \partial_{\xi}\left(\phi q_{J}\right)\right]
\end{aligned}
$$

Estimating $I_{i}, i=1, \ldots, 6$.

Estimate of $I_{1}$. Using Plancherel and Hölder's inequality, we observe

$$
\begin{aligned}
& \left\|I_{1}\right\|_{L_{t, x}^{2}}^{2}=\frac{1}{2 \pi(\delta K)^{2}}\left\|\int_{\mathbb{R}} \mathcal{F}_{t x}^{-1}\left[\tilde{\psi} \hat{\chi}_{J}(0, n, \xi)\right] \eta(\xi) \mathrm{d} \xi\right\|_{L_{t, x}^{2}}^{2} \\
& =\frac{1}{2 \pi(\delta K)^{2}} \int_{u} \sum_{n}\left|\int_{\mathbb{R}} \tilde{\psi} \hat{\chi}_{J}(0, n, \xi) \eta(\xi) \mathrm{d} \xi\right|^{2} \mathrm{~d} u \\
& \leq \frac{1}{2 \pi(\delta K)^{2}} \int_{u} \sum_{n} \int_{\mathbb{R}}\left|\tilde{\psi}\left(\frac{\mathcal{L}(i u, i n, \xi)}{\delta K}\right)\right|^{2} \mathbf{1}_{\text {supp } \eta} \mathrm{d} \xi \\
& \times \int_{\mathbb{R}} \mathbf{1}_{\left\{|u+b(\xi) \cdot n|^{2}+\left|n^{*} A(\xi) n\right|^{2}<(2 \delta K)^{2}\right\}}\left|\hat{\chi}_{J}(0, n, \xi)\right|^{2} \eta^{2}(\xi) \mathrm{d} \xi \mathrm{d} u .
\end{aligned}
$$

Then using (2.3) and

$$
\int_{u} \mathbf{1}_{\left\{|u+b(\xi) \cdot n|^{2}+\left|n^{*} A(\xi) n\right|^{2}<(2 \delta K)^{2}\right\}} \mathrm{d} u \leq \int_{u} \mathbf{1}_{\left\{|u|^{2}<(2 \delta K)^{2}\right\}} \mathrm{d} u \lesssim \delta K
$$

we obtain

$$
\left\|I_{1}\right\|_{L_{t, x}^{2}}^{2} \lesssim \frac{1}{\delta K}\left(\frac{\delta K}{J^{\beta}}\right)^{\alpha}\left\|\chi_{J}(0) \eta\right\|_{L_{x, \xi}^{2}}^{2}
$$


Estimate of $I_{2}$. First, we integrate by parts to obtain

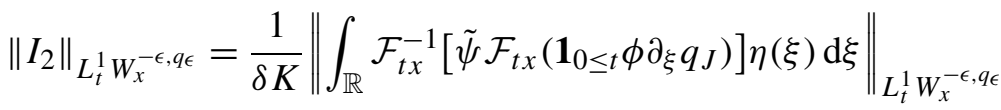

$$
\begin{aligned}
& \leq \frac{1}{(\delta K)^{2}} \| \int_{\mathbb{R}} \mathcal{F}_{t x}^{-1}\left[\tilde{\psi}^{\prime} \frac{\mathcal{L}_{\xi}(i u, i n, \xi)}{\vartheta(\xi)}\right. \\
& \left.\times \mathcal{F}_{t x}\left(\mathbf{1}_{0 \leq t} \phi \vartheta q_{J}\right)(u, \xi, n)\right] \eta(\xi) \mathrm{d} \xi \|_{L_{t}^{1} W_{x}^{-\epsilon, q_{\epsilon}}} \\
& +\frac{1}{(\delta K)^{2}}\left\|\int_{\mathbb{R}} \mathcal{F}_{t x}^{-1}\left[\tilde{\psi} \mathcal{F}_{t x}\left(\mathbf{1}_{0 \leq t} \phi q_{J}\right)(u, \xi, n)\right] \eta^{\prime}(\xi) \mathrm{d} \xi\right\|_{L_{t}^{1} W_{x}^{-\epsilon, q_{\epsilon}}},
\end{aligned}
$$

with the convention $\tilde{\psi}^{\prime}=\tilde{\psi}^{\prime}\left(\frac{\mathcal{L}(i u, i n, \xi)}{\delta K}\right)$ and similarly for $\tilde{\psi}$. We apply Lemma A.1 to estimate the second term on the right-hand side. For the first one, we first note that (2.3) implies that (for simplicity restricting to the case $\beta=2$ while $\beta=1$ can be handled analogously)

$$
\frac{\left|b_{i}^{\prime}(\xi)\right|}{\vartheta(\xi)} \lesssim_{\eta}|n|, \quad \frac{\left|A_{i j}^{\prime}(\xi)\right|}{\vartheta(\xi)} \lesssim_{\eta} 1, \quad i, j \in\{1, \ldots, N\} .
$$

Since $\mathcal{L}_{\xi}(i u, i n, \xi)$ is a polynomial in $n$, we may apply [2], Lemma 2.2 , to deduce that

$$
m(n, \xi):=\frac{\mathcal{L}_{\xi}(i u, i n, \xi)}{\vartheta(\xi)}=\frac{i b^{\prime}(\xi) \cdot n+n^{*} A^{\prime}(\xi) n}{\vartheta(\xi)}
$$

localized to $|n| \sim J, \xi \in \operatorname{supp} \eta$, is an $L^{1}$-Fourier multiplier with norm bounded by $J^{\beta}$. Revisiting the proof of Lemma A.1 (see proof of [51], Lemma 2.2) with the multiplier $\psi\left(\frac{m(u, n, \xi)}{\delta}\right)$ replaced by $\psi\left(\frac{m(u, n, \xi)}{\delta}\right) m(n, \xi)$ then yields

$$
\left\|I_{2}\right\|_{L_{t}^{1} W_{x}^{-\epsilon, q_{\epsilon}}} \lesssim \frac{1}{(\delta K)^{2}} J^{\beta}\left\|\phi \vartheta q_{J}\left(\eta+\left|\eta^{\prime}\right|\right)\right\|_{\mathcal{M}_{t, x, \xi}},
$$

where $\frac{N}{q_{\epsilon}^{\prime}}<\epsilon<1<q_{\epsilon}<\frac{N}{N-\epsilon}$ and $\epsilon$ is chosen sufficiently small. Consequently,

$$
\left\|I_{2}\right\|_{L_{t, x}^{1}} \lesssim \frac{1}{(\delta K)^{2}} J^{\beta+\epsilon}\left\|\phi \vartheta q_{J}\left(\eta+\left|\eta^{\prime}\right|\right)\right\|_{\mathcal{M}_{t, x, \xi}} .
$$

Estimate of $I_{3}$. Using Plancherel and Itô's formula, we note that

$$
\begin{aligned}
\mathbb{E}\left\|I_{3}\right\|_{L_{t, x}^{2}}^{2} & \\
= & \frac{1}{2 \pi(\delta K)^{2}} \\
& \left.\times \mathbb{E} \| \int_{\mathbb{R}} \mathcal{F}_{t x}^{-1}\left[\tilde{\psi} \sum_{k=1}^{\infty} \int_{0}^{\infty} \mathrm{e}^{-i s u} \partial_{\xi} \widehat{\left(g_{k} \chi \phi\right.}\right)_{J}(s, \xi, n) \mathrm{d} \beta_{k}(s)\right] \eta(\xi) \mathrm{d} \xi \|_{L_{t, x}^{2}}^{2}
\end{aligned}
$$




$$
\begin{aligned}
= & \frac{1}{2 \pi(\delta K)^{2}} \\
& \left.\times \int_{u} \sum_{n} \mathbb{E} \mid \sum_{k=1}^{\infty} \int_{0}^{\infty} \int_{\mathbb{R}} \tilde{\psi} \mathrm{e}^{-i s u} \partial_{\xi} \widehat{\left(g_{k} \chi \phi\right.}\right)\left._{J}(s, \xi, n) \eta(\xi) \mathrm{d} \xi \mathrm{d} \beta_{k}(s)\right|^{2} \mathrm{~d} u \\
= & \frac{1}{2 \pi(\delta K)^{2}} \\
& \left.\times \int_{u} \sum_{n} \mathbb{E} \int_{0}^{\infty} \sum_{k=1}^{\infty} \mid \int_{\mathbb{R}} \tilde{\psi} \mathrm{e}^{-i s u} \partial_{\xi} \widehat{\left(g_{k} \chi \phi\right.}\right)\left._{J}(s, \xi, n) \eta(\xi) \mathrm{d} \xi\right|^{2} \mathrm{~d} s \mathrm{~d} u \\
\lesssim & \frac{1}{(\delta K)^{4}} \\
& \left.\times \int_{u} \sum_{n} \mathbb{E} \int_{0}^{\infty} \sum_{k=1}^{\infty} \mid \int_{\mathbb{R}} \tilde{\psi}^{\prime} \frac{\mathcal{L}_{\xi}(i u, i n, \xi)}{\vartheta(\xi)} \widehat{\left(g_{k} \chi \vartheta \phi\right.}\right)\left._{J}(s, \xi, n) \eta(\xi) \mathrm{d} \xi\right|^{2} \mathrm{~d} s \mathrm{~d} u \\
& \left.+\frac{1}{(\delta K)^{4}} \int_{u} \sum_{n} \mathbb{E} \int_{0}^{\infty} \sum_{k=1}^{\infty} \mid \int_{\mathbb{R}} \tilde{\psi} \widehat{\left(g_{k} \chi \phi\right.}\right)\left._{J}(s, \xi, n) \eta^{\prime}(\xi) \mathrm{d} \xi\right|^{2} \mathrm{~d} s \mathrm{~d} u
\end{aligned}
$$

Hence, by (2.3) it follows that

$$
\begin{aligned}
\mathbb{E}\left\|I_{3}\right\|_{L_{t, x}^{2}}^{2} & \lesssim \\
\lesssim & \frac{1}{(\delta K)^{4}} \mathbb{E} \int_{0}^{\infty} \int_{u} \sum_{n} \int_{\mathbb{R}}\left|\tilde{\psi}^{\prime} \frac{\mathcal{L}_{\xi}(i u, i n, \xi)}{\vartheta(\xi)}\right|^{2} \mathbf{1}_{\text {supp } \eta} \mathrm{d} \xi \\
& \times \int_{\mathbb{R}} \mathbf{1}_{\left\{|u+b(\xi) \cdot n|^{2}+\left|n^{*} A(\xi) n\right|^{2}<(2 \delta K)^{2}\right\}} \\
& \left.\times \sum_{k=1}^{\infty} \mid \widehat{\left(g_{k} \chi \vartheta \phi\right.}\right)\left._{J}(s, \xi, n)\right|^{2} \eta^{2} \mathrm{~d} \xi \mathrm{d} u \mathrm{~d} s \\
& +\frac{1}{(\delta K)^{4}} \mathbb{E} \int_{0}^{\infty} \int_{u} \sum_{n} \int_{\mathbb{R}}\left|\tilde{\psi}\left(\frac{\mathcal{L}(i u, i n, \xi)}{\delta K}\right)\right|^{2} \mathbf{1}_{\text {supp } \eta} \mathrm{d} \xi \\
& \times \int_{\mathbb{R}} \mathbf{1}_{\left\{|u+b(\xi) \cdot n|^{2}+\left|n^{*} A(\xi) n\right|^{2}<(2 \delta K)^{2}\right\}} \\
& \times \sum_{k=1}^{\infty}\left|\left(\widehat{g_{k} \chi \vartheta \phi}\right)_{J}(s, \xi, n)\right|^{2}\left|\eta^{\prime}\right|^{2} \mathrm{~d} \xi \mathrm{d} u \mathrm{~d} s \\
\lesssim & \frac{1}{(\delta K)^{4}}\left(\frac{\delta K}{J^{\beta}}\right)^{\alpha} J^{2 \beta} \mathbb{E} \int_{0}^{\infty} \int_{u} \sum_{n} \int_{\mathbb{R}} \mathbf{1}_{\left\{|u+b(\xi) \cdot n|^{2}+\left|n^{*} A(\xi) n\right|^{2}<(2 \delta K)^{2}\right\}}
\end{aligned}
$$




$$
\begin{aligned}
& \times \sum_{k=1}^{\infty}\left|\left(\widehat{g_{k} \chi \vartheta \phi}\right)_{J}(s, \xi, n)\right|^{2} \eta^{2} \mathrm{~d} \xi \mathrm{d} u \mathrm{~d} s \\
& +\frac{1}{(\delta K)^{4}}\left(\frac{\delta K}{J^{\beta}}\right)^{\alpha} \mathbb{E} \int_{0}^{\infty} \int_{u} \sum_{n} \int_{\mathbb{R}} \mathbf{1}_{\left\{|u+b(\xi) \cdot n|^{2}+\left|n^{*} A(\xi) n\right|^{2}<(2 \delta K)^{2}\right\}} \\
& \left.\times \sum_{k=1}^{\infty} \mid \widehat{\left(g_{k} \chi \vartheta \phi\right.}\right)\left._{J}(s, \xi, n)\right|^{2}\left|\eta^{\prime}\right|^{2} \mathrm{~d} \xi \mathrm{d} u \mathrm{~d} s
\end{aligned}
$$

and due to (3.5) we obtain

$$
\mathbb{E}\left\|I_{3}\right\|_{L_{t, x}^{2}}^{2} \leq \frac{1}{(\delta K)^{3}}\left(\frac{\delta K}{J^{\beta}}\right)^{\alpha} J^{2 \beta} \sum_{k=1}^{\infty} \mathbb{E}\left\|\left(g_{k} \chi \vartheta \phi\right)_{J}\left(\eta+\left|\eta^{\prime}\right|\right)\right\|_{L_{t, x, \xi}^{2}}^{2} .
$$

Estimate of $I_{4}$. By Plancherel and Itô's formula, we have $\mathbb{E}\left\|I_{4}\right\|_{L_{t, x}^{2}}^{2}$

$$
\begin{aligned}
\lesssim & \frac{1}{(\delta K)^{2}} \mathbb{E} \| \int_{\mathbb{R}} \mathcal{F}_{t x}^{-1} \\
& \times\left[\tilde{\psi} \sum_{k=1}^{\infty} \int_{0}^{\infty} \mathrm{e}^{-i s u} \mathcal{F}_{x}\left(\left(\partial_{\xi} g_{k}\right) \chi \phi\right)_{J}(s, \xi, n) \mathrm{d} \beta_{k}(s)\right] \eta(\xi) \mathrm{d} \xi \|_{L_{t, x}^{2}}^{2} \\
= & \frac{1}{(\delta K)^{2}} \\
& \times \int_{u} \sum_{n} \mathbb{E}\left|\sum_{k=1}^{\infty} \int_{0}^{\infty} \int_{\mathbb{R}} \tilde{\psi} \mathrm{e}^{-i s u} \mathcal{F}_{x}\left(\left(\partial_{\xi} g_{k}\right) \chi \phi\right)_{J}(s, \xi, n) \eta(\xi) \mathrm{d} \xi \mathrm{d} \beta_{k}(s)\right|^{2} \mathrm{~d} u \\
= & \frac{1}{(\delta K)^{2}} \int_{u} \sum_{n} \mathbb{E} \\
& \times \int_{0}^{\infty} \sum_{k=1}^{\infty}\left|\int_{\mathbb{R}} \tilde{\psi} \mathrm{e}^{-i s u} \mathcal{F}_{x}\left(\left(\partial_{\xi} g_{k}\right) \chi \phi\right)_{J}(s, \xi, n) \eta(\xi) \mathrm{d} \xi\right|^{2} \mathrm{~d} s \mathrm{~d} u .
\end{aligned}
$$

Thus, it follows from (2.3), (3.5) and (2.4) that

$$
\begin{aligned}
\mathbb{E}\left\|I_{4}\right\|_{L_{t, x}^{2}}^{2} & \lesssim \frac{1}{(\delta K)^{2}} \int_{u} \sum_{n} \int_{\mathbb{R}}\left|\tilde{\psi}\left(\frac{\mathcal{L}(i u, i n, \xi)}{\delta K}\right)\right|^{2} \mathbf{1}_{\operatorname{supp} \eta} \mathrm{d} \xi \\
& \times \mathbb{E} \int_{0}^{\infty} \int_{\mathbb{R}} \mathbf{1}_{\left\{|u+b(\xi) \cdot n|^{2}+\left|n^{*} A(\xi) n\right|^{2}<(2 \delta K)^{2}\right\}}
\end{aligned}
$$




$$
\begin{aligned}
& \times \sum_{k=1}^{\infty}\left|\mathcal{F}_{x}\left(\left(\partial_{\xi} g_{k}\right) \chi \phi\right)_{J}(s, \xi, n)\right|^{2} \eta^{2} \mathrm{~d} \xi \mathrm{d} s \mathrm{~d} u \\
\lesssim & \frac{1}{\delta K}\left(\frac{\delta K}{J^{\beta}}\right)^{\alpha} \sum_{k=1}^{\infty} \mathbb{E}\left\|\left(\left(\partial_{\xi} g_{k}\right) \chi \eta \phi\right)_{J}\right\|_{L_{t, x, \xi}^{2}}^{2} .
\end{aligned}
$$

Estimate of $I_{5}$. We have, using (3.5),

$\mathbb{E}\left\|I_{5}\right\|_{L_{t, x}^{2}}^{2}$

$$
\begin{aligned}
& =\frac{1}{2 \pi(\delta K)^{2}} \mathbb{E} \| \int_{\mathbb{R}} \mathcal{F}_{t x}^{-1} \\
& \times\left[\tilde{\psi} \sum_{k=1}^{\infty} \int_{0}^{\infty} \mathrm{e}^{-i s u} \delta_{0} \phi \widehat{g_{k, J}}(n, \xi) \mathrm{d} \beta_{k}(s)\right] \eta(\xi) \mathrm{d} \xi \|_{L_{t, x}^{2}}^{2} \\
& =\frac{1}{2 \pi(\delta K)^{2}} \mathbb{E}\left\|\int_{\mathbb{R}} \tilde{\psi} \sum_{k=1}^{\infty} \int_{0}^{\infty} \mathrm{e}^{-i s u} \delta_{0} \phi \widehat{g_{k, J}}(n, \xi) \mathrm{d} \beta_{k}(s) \eta(\xi) \mathrm{d} \xi\right\|_{L_{u, n}^{2}}^{2} \\
& =\frac{\eta(0)^{2}}{2 \pi(\delta K)^{2}} \int_{u} \sum_{n} \mathbb{E} \\
& \times\left|\sum_{k=1}^{\infty} \int_{0}^{\infty} \tilde{\psi}\left(\frac{\mathcal{L}(i u, i n, 0)}{\delta K}\right) \mathrm{e}^{-i s u} \phi \widehat{g_{k, J}}(n, 0) \mathrm{d} \beta_{k}(s)\right|^{2} \mathrm{~d} u \\
& =\frac{\eta(0)^{2}}{2 \pi(\delta K)^{2}} \int_{u} \sum_{n} \int_{0}^{\infty} \sum_{k=1}^{\infty}\left|\tilde{\psi}\left(\frac{\mathcal{L}(i u, i n, 0)}{\delta K}\right) \phi \widehat{g_{k, J}}(n, 0)\right|^{2} \mathrm{~d} s \mathrm{~d} u \\
& \lesssim \eta \frac{1}{\delta K} \sum_{k=1}^{\infty}\left\|g_{k, J}(\cdot, 0)\right\|_{L_{x}^{2}}^{2} .
\end{aligned}
$$

Estimate of $I_{6}$. It holds

$$
\begin{aligned}
\left\|I_{6}\right\|_{L_{t, x}^{1}} & \lesssim \frac{1}{\delta K}\left\|\int_{\mathbb{R}} \mathcal{F}_{t x}^{-1}\left[\tilde{\psi} \mathcal{F}_{t x}\left(\mathbf{1}_{0 \leq t} \chi_{J} \partial_{t} \phi\right)\right] \eta(\xi) \mathrm{d} \xi\right\|_{L_{t, x}^{1}} \\
& \lesssim \frac{1}{\delta K}\left\|\partial_{t} \phi \chi_{J} \eta\right\|_{L_{t, x, \xi}^{1}} .
\end{aligned}
$$

Estimating $\chi_{J}^{(K)}$. Let us denote

$$
\begin{aligned}
\mathcal{K}:= & \left\|\chi_{J}(0) \eta\right\|_{L_{\omega, x, \xi}^{2}}+\left\|\phi q_{J} \vartheta\left(\eta+\left|\eta^{\prime}\right|\right)\right\|_{L_{\omega}^{1} \mathcal{M}_{t, x, \xi}} \\
& +\left(\sum_{k=1}^{\infty}\left\|\left(g_{k} \chi \vartheta \phi\right)_{J}\left(\eta+\left|\eta^{\prime}\right|\right)\right\|_{L_{\omega, t, x, \xi}^{2}}^{2}\right)^{1 / 2}
\end{aligned}
$$




$$
\begin{aligned}
& +\left(\sum_{k=1}^{\infty}\left\|\left(\left(\partial_{\xi} g_{k}\right) \chi \eta \phi\right)_{J}\right\|_{L_{\omega, t, x, \xi}^{2}}^{2}\right)^{1 / 2} \\
& +\left(\sum_{k=1}^{\infty}\left\|g_{k, J}(\cdot, 0)\right\|_{L_{x}^{2}}^{2}\right)^{1 / 2}+\left\|\partial_{t} \phi \chi_{J} \eta\right\|_{L_{\omega, t, x, \xi}^{1}} .
\end{aligned}
$$

Then using (3.6), (3.7), (3.8), (3.9), (3.10), (3.11) we deduce

$$
\begin{aligned}
& \left\|\int_{\mathbb{R}} \chi_{J}^{(K)} \eta \mathrm{d} \xi\right\|_{L_{\omega, t, x}^{1}} \\
& =\sup _{\substack{\varphi \in L_{\omega, t, x}^{\infty} \\
\|\varphi\|_{L_{\omega, t, x}^{\infty}}^{\infty}}} \mathbb{E}\left\langle\int_{\mathbb{R}} \chi_{J}^{(K)} \eta \mathrm{d} \xi, \varphi\right) \\
& \lesssim \eta \mathcal{K}\left[\left(\frac{1}{\delta K}\right)^{\frac{1}{2}}\left(\frac{\delta K}{J^{\beta}}\right)^{\frac{\alpha}{2}}+\frac{1}{(\delta K)^{2}} J^{\beta+\epsilon}+\left(\frac{1}{(\delta K)^{3}}\right)^{\frac{1}{2}}\left(\frac{\delta K}{J^{\beta}}\right)^{\frac{\alpha}{2}} J^{\beta}\right. \\
& \left.+\left(\frac{1}{\delta K}\right)^{\frac{1}{2}}\left(\frac{\delta K}{J^{\beta}}\right)^{\frac{\alpha}{2}}+\left(\frac{1}{\delta K}\right)^{\frac{1}{2}}+\frac{1}{\delta K}\right] .
\end{aligned}
$$

Now summing over all dyadic $K$, since all the powers of $K$ appearing on the righthand side are negative, we deduce

$$
\begin{gathered}
\sum_{K \gtrsim 1}\left\|\int_{\mathbb{R}} \chi_{J}^{(K)} \eta \mathrm{d} \xi\right\|_{L_{\omega, t, x}^{1}} \lesssim \\
\mathcal{K}\left[\delta^{\frac{1}{2}(\alpha-1)} J^{-\frac{\beta \alpha}{2}}+\delta^{-2} J^{\beta+\epsilon}+\delta^{-\frac{3-\alpha}{2}} J^{\frac{2-\alpha}{2} \beta}\right. \\
\left.+\delta^{-\frac{1}{2}(1-\alpha)} J^{-\frac{\beta \alpha}{2}}+\delta^{-\frac{1}{2}}+\delta^{-1}\right] .
\end{gathered}
$$

Estimating $\chi_{J}$. We let $\overline{\chi_{J} \phi \eta}:=\int_{\mathbb{R}} \chi_{J} \phi \eta \mathrm{d} \xi$ and write

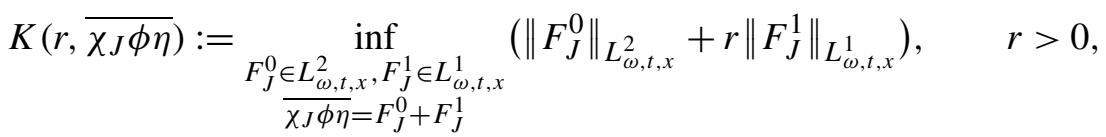

where $\overline{\chi_{J} \phi \eta}=F_{J}^{0}+F_{J}^{1}$ with $F_{J}^{0}:=\int_{\mathbb{R}} \chi_{J}^{(0)} \eta \mathrm{d} \xi, F_{J}^{1}:=\sum_{K \gtrsim 1} \int_{\mathbb{R}} \chi_{J}^{(K)} \eta \mathrm{d} \xi$. By Lemma A.1 and (2.3), we have

$$
\begin{aligned}
\left\|F_{J}^{0}\right\|_{L_{t, x}^{2}}^{2} & =\left\|\int_{\mathbb{R}} \mathcal{F}_{t x}^{-1}\left[\psi_{0}\left(\frac{\mathcal{L}(i u, i n, \xi)}{\delta}\right) \mathcal{F}_{t x}\left(\chi_{J} \phi\right)(u, n, \xi)\right] \eta(\xi) \mathrm{d} \xi\right\|_{L_{t, x}^{2}}^{2} \\
& \lesssim\left(\frac{\delta}{J^{\beta}}\right)^{\alpha}\left\|\chi_{J} \eta \phi\right\|_{L_{t, x, \xi}^{2}}^{2} .
\end{aligned}
$$

Hence, we obtain

$$
\begin{aligned}
K\left(r, \overline{\chi_{J} \phi \eta}\right) \lesssim & \delta^{\frac{\alpha}{2}} J^{-\frac{\beta \alpha}{2}}\left\|\chi_{J} \eta \phi\right\|_{L_{\omega, t, x, \xi}^{2}}+r \mathcal{K}\left[\delta^{\frac{1}{2}(\alpha-1)} J^{-\frac{\beta \alpha}{2}}+\delta^{-2} J^{\beta+\epsilon}\right. \\
& \left.+\delta^{-\frac{3-\alpha}{2}} J^{\frac{2-\alpha}{2} \beta}+\delta^{-\frac{1}{2}(1-\alpha)} J^{-\frac{\beta \alpha}{2}}+\delta^{-\frac{1}{2}}+\delta^{-1}\right]
\end{aligned}
$$


and we intend to choose $r$ to equilibrate these bounds. To do so, let $\tau, \kappa>0$ to be chosen later and set $\delta=r^{\tau} J^{\kappa}$. This yields

$$
\begin{aligned}
r^{-\tau \frac{\alpha}{2}} K\left(r, \overline{\chi_{J} \phi \eta}\right) \lesssim & J^{\alpha \frac{\kappa-\beta}{2}}\left\|\chi_{J} \eta \phi\right\|_{L_{\omega, t, x, \xi}^{2}} \\
& +\mathcal{K}\left[r^{1-\frac{\tau}{2}} J^{\frac{-\kappa(1-\alpha)-\beta \alpha}{2}}+r^{1-\tau\left(\frac{4+\alpha}{2}\right)} J^{-2 \kappa+\beta+\epsilon}\right. \\
& \left.+r^{1-\frac{3}{2} \tau} J^{-\frac{3-\alpha}{2} \kappa+\frac{2-\alpha}{2} \beta}+r^{1-\tau\left(\frac{1+\alpha}{2}\right)} J^{-\frac{1}{2} \kappa}+r^{1-\tau\left(\frac{2+\alpha}{2}\right)} J^{-\kappa}\right] .
\end{aligned}
$$

Optimizing in $\tau, \kappa$ yields $\kappa=\frac{2}{3} \beta, \tau=\frac{2}{4+\alpha}$. which obviously can be satisfied. Hence, with $\theta:=\frac{\alpha}{4-\alpha}$,

$$
\begin{aligned}
J^{\frac{\alpha \beta}{6}} r^{\theta} K\left(r, \overline{\chi_{J} \phi \eta}\right) \lesssim & \left\|\chi_{J} \eta \phi\right\|_{L_{\omega, t, x, \xi}^{2}}+\mathcal{K}\left[r^{\frac{3+\alpha}{4+\alpha}} J^{-\frac{1}{3} \beta}+J^{-\frac{2-\alpha}{6} \beta+\epsilon}+r^{\frac{1+\alpha}{4+\alpha}}\right. \\
& \left.+r^{\frac{3}{4+\alpha}} J^{-\frac{2-\alpha}{6} \beta}+r^{\frac{2}{4+\alpha}} J^{-\frac{4-\alpha}{6} \beta}\right] .
\end{aligned}
$$

Finally, since for $r$ large we have the elementary estimate

$$
K\left(r, \overline{\chi_{J} \phi \eta}\right) \leq\left\|\overline{\chi_{J} \phi \eta}\right\|_{L_{\omega, t, x}^{2}}
$$

we apply the K-method of real interpolation to deduce

$$
\begin{aligned}
& J^{\frac{\alpha \beta}{6}}\left\|\overline{\chi_{J} \phi \eta}\right\|_{\left(L_{\omega, t, x}^{2}, L_{\omega, t, x}^{1}\right)_{\theta, \infty}} \leq J^{\frac{\alpha \beta}{6}}\left\|r^{-\theta} K\left(r, \overline{\chi_{J} \phi \eta}\right)\right\|_{L_{r}^{\infty}} \\
& \leq J^{\frac{\alpha \beta}{6}}\left\|r^{-\theta} K\left(r, \overline{\chi_{J} \phi \eta}\right) \mathbf{1}_{r \leq R}\right\|_{L_{r}^{\infty}}+J^{\frac{\alpha \beta}{6}}\left\|r^{-\theta} K\left(r, \overline{\chi_{J} \phi \eta}\right) \mathbf{1}_{r \geq R}\right\|_{L_{r}^{\infty}} \\
& \lesssim\left\|\chi_{J} \phi \eta\right\|_{L_{\omega, t, x, \xi}^{2}}+\mathcal{K}\left[R^{\frac{3+\alpha}{4+\alpha}} J^{-\frac{1}{3} \beta}+J^{-\frac{2-\alpha}{6} \beta+\epsilon}+R^{\frac{1+\alpha}{4+\alpha}}\right. \\
&\left.\quad+R^{\frac{3}{4+\alpha}} J^{-\frac{2-\alpha}{6} \beta}+R^{\frac{2}{4+\alpha}} J^{-\frac{4-\alpha}{6} \beta}\right]+J^{\frac{\alpha \beta}{6}} R^{-\theta}\left\|\overline{\chi_{J} \phi \eta}\right\|_{L_{\omega, t, x}^{2}} .
\end{aligned}
$$

Let us take $R=J^{\tau}$ for some $\tau>0$ to be chosen below. Then

$$
\begin{aligned}
J^{\frac{\alpha \beta}{6}}\left\|\overline{\chi_{J} \phi \eta}\right\|_{\left(L_{\omega, t, x}^{2}, L_{\omega, t, x}^{1}\right)_{\theta, \infty}} & \\
\lesssim & \left\|\chi_{J} \phi \eta\right\|_{L_{\omega, t, x, \xi}^{2}}+\mathcal{K}\left[\left(J^{\tau}\right)^{\frac{3+\alpha}{4+\alpha}} J^{-\frac{1}{3} \beta}+J^{-\frac{2-\alpha}{6} \beta+\epsilon}+\left(J^{\tau}\right)^{\frac{1+\alpha}{4+\alpha}}\right. \\
& \left.+\left(J^{\tau}\right)^{\frac{3}{4+\alpha}} J^{-\frac{2-\alpha}{6} \beta}+\left(J^{\tau}\right)^{\frac{2}{4+\alpha}} J^{-\frac{4-\alpha}{6} \beta}\right] \\
& +J^{\frac{\alpha \beta}{6}}\left(J^{\tau}\right)^{-\theta}\left\|\overline{\chi_{J} \phi \eta}\right\|_{L_{\omega, t, x}^{2}} .
\end{aligned}
$$

Now we aim to choose $\tau$ in order to minimize the maximum of the exponents of $J$ occurring on the right-hand side in the previous inequality. Optimizing for $\tau$ the 
terms

$$
\begin{aligned}
& \frac{3+\alpha}{4+\alpha} \tau-\frac{\beta}{3}, \quad \frac{1+\alpha}{4+\alpha} \tau, \quad \frac{3}{4+\alpha} \tau-\frac{2-\alpha}{6} \beta, \\
& \frac{2}{4+\alpha} \tau-\frac{4-\alpha}{6} \beta, \quad-\frac{\alpha}{4+\alpha} \tau+\frac{\alpha \beta}{6}
\end{aligned}
$$

yields the choice $\tau=\frac{\beta \alpha(4+\alpha)}{6(1+2 \alpha)}$. With this choice, all the exponents in the righthand side of (3.12) are of order less than $\frac{\alpha \beta}{6}$. Multiplication with $J^{\frac{-\alpha \beta}{6}}$ thus leads to negative powers of $J$ on the right-hand side, the worst (i.e., the maximal) one being $-\frac{\alpha^{2} \beta}{6(1+2 \alpha)}$. Therefore, since $\epsilon$ was chosen small, we obtain that

$$
\left\|\overline{\chi_{J} \phi \eta}\right\|_{\left(L_{\omega, t, x}^{2}, L_{\omega, t, x}^{1}\right)_{\theta, \infty}} \lesssim J^{-\frac{\alpha^{2} \beta}{(1+2 \alpha)}}\left[\left\|\chi_{J} \phi \eta\right\|_{L_{\omega, t, x, \xi}^{2}}+\mathcal{K}+\left\|\overline{\chi_{J} \phi \eta}\right\|_{L_{\omega, t, x}^{2}}\right] .
$$

Note that although all the above norms are global in time, that is, $t \in(-\infty, \infty)$, the integrands are localized on $[0, T]$ due to the cut-off $\phi=\phi^{\lambda}$.

Conclusion. The real interpolation of two Lebesgue spaces is given by a Lorentz space (see [52], Section 1.18.6, Theorem 1, namely,

$$
\left(L_{\omega, t, x}^{2}, L_{\omega, t, x}^{1}\right)_{\theta, \infty}=L_{\omega, t, x}^{\tilde{r}, \infty}, \quad \frac{1}{\tilde{r}}=\frac{1-\theta}{2}+\frac{\theta}{1} .
$$

In the case of a bounded domain, $L_{\omega, t, x}^{\tilde{r}, \infty}$ is embedded in the Lebesgue space $L_{\omega, t, x}^{r}$ whenever $\tilde{r}>r$ (see [31], Exercise 1.1.11),

$$
\left(L_{\omega, t, x}^{2}, L_{\omega, t, x}^{1}\right)_{\theta, \infty} \hookrightarrow L_{\omega, t, x}^{r}, \quad \frac{1}{r}>\frac{1-\theta}{2}+\frac{\theta}{1} .
$$

Thus letting $s<\frac{\alpha^{2} \beta}{6(1+2 \alpha)}$ we deduce

$$
\begin{aligned}
\|\overline{\chi \phi \eta}\|_{L^{r}\left(\Omega \times[0, T] ; W^{s, r}\left(\mathbb{T}^{N}\right)\right)} & \vdots\|\chi \phi \eta\|_{L_{\omega, t, x, \xi}^{2}}+\|\chi(0) \eta\|_{L_{\omega, x, \xi}^{2}} \\
& +\left(\sum_{k=1}^{\infty}\left\|\left(\left(\partial_{\xi} g_{k}\right) \chi \eta \phi\right)\right\|_{L_{\omega, t, x, \xi}^{2}}^{2}\right)^{1 / 2} \\
& +\left(\sum_{k=1}^{\infty}\left\|g_{k} \chi \vartheta \phi\left(\eta+\left|\eta^{\prime}\right|\right)\right\|_{L_{\omega, t, x, \xi}^{2}}^{2}\right)^{1 / 2} \\
& +\left(\sum_{k=1}^{\infty}\left\|g_{k}(\cdot, 0)\right\|_{L_{x}^{2}}^{2}\right)^{1 / 2} \\
& +\left\|\phi \vartheta q\left(\eta+\left|\eta^{\prime}\right|\right)\right\|_{L_{\omega}^{1} \mathcal{M}_{t, x, \xi}}+\left\|\partial_{t} \phi \chi \eta\right\|_{L_{\omega, t, x, \xi}^{1}} \\
& +\|\overline{\chi \phi \eta}\|_{L_{\omega, t, x}^{2} \cdot}
\end{aligned}
$$


Since the constant in inequality (3.13) is independent of $\lambda$, we may send $\lambda \rightarrow 0$. First, we observe that

$$
\limsup _{\lambda \rightarrow 0}\left\|\partial_{t} \phi^{\lambda} \chi \eta\right\|_{L_{\omega, t, x, \xi}^{1}} \lesssim \sup _{0 \leq t \leq T}\|\chi \eta\|_{L_{\omega, x, \xi}^{1}}
$$

Using the dominated convergence theorem for the remaining terms, we obtain the following estimate for $u^{\eta}=\overline{\chi \eta}$ :

$$
\begin{aligned}
& \left\|u^{\eta}\right\|_{L^{r}\left(\Omega \times[0, T] ; W^{s, r}\left(\mathbb{T}^{N}\right)\right)} \\
& \left.\lesssim\|\chi \eta\|_{L_{\omega, t, x, \xi}^{2}}+\|\chi(0) \eta\|_{L_{\omega, x, \xi}^{2}}\right)^{1 / 2}+\left(\sum_{k=1}^{\infty}\left\|g_{k} \chi \vartheta\left(\eta+\left|\eta^{\prime}\right|\right)\right\|_{L_{\omega, t, x, \xi}^{2}}^{2}\right)^{1 / 2} \\
& \quad+\left(\sum_{k=1}^{\infty}\left\|\left(\partial_{\xi} g_{k}\right) \chi \eta\right\|_{L_{\omega, t, x, \xi}^{2}}^{2}\right. \\
& \quad+\left(\sum_{k=1}^{\infty}\left\|g_{k}(\cdot, 0)\right\|_{L_{x}^{2}}^{2}\right)^{1 / 2}+\left\|q \vartheta\left(\eta+\left|\eta^{\prime}\right|\right)\right\|_{L_{\omega}^{1} \mathcal{M}_{t, x, \xi}}+\sup _{0 \leq t \leq T}\|\chi \eta\|_{L_{\omega, x, \xi}^{1}} \\
& \quad+\|\bar{\chi} \eta\|_{L_{\omega, t, x}^{2}} .
\end{aligned}
$$

It remains to estimate the right-hand side in terms of the available bounds for the kinetic solution $u$ and the corresponding kinetic measure $m$. Note that this estimate will depend on the localization $\eta$. First, due to the definition of the equilibrium function $\chi$, it follows immediately that

$$
\begin{aligned}
& \|\chi \eta\|_{L_{\omega, t, x, \xi}^{2}} \lesssim \eta\left\|u^{\eta}\right\|_{L_{\omega, t, x}^{1}}^{1 / 2}, \quad\|\chi(0) \eta\|_{L_{\omega, x, \xi}^{2}} \lesssim \eta\left\|u_{0}^{\eta}\right\|_{L_{\omega, x}^{1}}^{1 / 2}, \\
& \sup _{0 \leq t \leq T}\|\chi \eta\|_{L_{\omega, x, \xi}^{1}}=\sup _{0 \leq t \leq T}\left\|u^{\eta}\right\|_{L_{\omega, x}^{1}}, \quad\|\bar{\chi} \eta\|_{L_{\omega, t, x}^{2}} \lesssim_{\eta}\left\|u^{\eta}\right\|_{L_{\omega, t, x}^{1}}^{1 / 2} .
\end{aligned}
$$

Second, similarly and due to (2.4) we have with $\Theta_{\eta}$ such that $\Theta_{\eta}^{\prime}=\left(|\xi|^{2}+\right.$ 1) $\vartheta^{2}(\xi)\left(\eta(\xi)+\left|\eta^{\prime}\right|(\xi)\right)$,

$$
\begin{gathered}
\left(\sum_{k=1}^{\infty}\left\|\left(\partial_{\xi} g_{k}\right) \chi \eta\right\|_{L_{\omega, t, x, \xi}^{2}}^{2}\right)^{1 / 2}+\left(\sum_{k=1}^{\infty}\left\|g_{k} \chi \vartheta\left(\eta+\left|\eta^{\prime}\right|\right)\right\|_{L_{\omega, t, x, \xi}^{2}}^{2}\right)^{1 / 2} \\
+\left(\sum_{k=1}^{\infty}\left\|g_{k}(\cdot, 0)\right\|_{L_{x}^{2}}^{2}\right)^{1 / 2} \lesssim \eta\left\|\Theta_{\eta}(u)\right\|_{L_{\omega, t, x}^{1}}^{1 / 2}+1
\end{gathered}
$$

Finally, since $q=m-\frac{1}{2} G^{2} \delta_{u=\xi}$, we deduce

$$
\left\|q \vartheta\left(\eta+\left|\eta^{\prime}\right|\right)\right\|_{L_{\omega}^{1} \mathcal{M}_{t, x, \xi}} \lesssim \eta\left\|m \vartheta\left(\eta+\left|\eta^{\prime}\right|\right)\right\|_{L_{\omega}^{1} \mathcal{M}_{t, x, \xi}}+1
$$


At this point, it is worth noticing that all estimates were uniform in $\eta$ up to constants depending on $\|\eta\|_{C^{1}}$ and (2.3). Since $\Theta_{\eta}(u) \geq u^{\eta}$, we conclude

$$
\begin{aligned}
& \|\bar{\eta}(u)\|_{L^{r}\left(\Omega \times[0, T] ; W^{s, r}\left(\mathbb{T}^{N}\right)\right)} \\
& \quad \lesssim_{\eta}\left\|\bar{\eta}\left(\left|u_{0}\right|\right)\right\|_{L_{\omega, x}^{1}}^{1 / 2}+\left\|\Theta_{\eta}(|u|)\right\|_{L_{\omega, t, x}^{1}}^{1 / 2} \\
& \quad+\sup _{0 \leq t \leq T}\|\bar{\eta}(|u|)\|_{L_{\omega, x}^{1}}+\left\|m \vartheta\left(\eta+\left|\eta^{\prime}\right|\right)\right\|_{L_{\omega}^{1} \mathcal{M}_{t, x, \xi}}+1 .
\end{aligned}
$$

with $\frac{1}{r}>\frac{1-\theta}{2}+\frac{\theta}{1}$ and $s<\frac{\alpha^{2} \beta}{6(1+2 \alpha)}$ which completes the proof.

4. Well-posedness. In this section, we present the proof of the main wellposedness result Theorem 1.1. The uniqueness part of Theorem 1.1 will be proved in Theorem 4.3 below, the existence in Theorem 4.9 below.

4.1. Uniqueness. In this section, we prove comparison results, and thus uniqueness for kinetic solutions to (1.1). We emphasize that we do not assume any higher $L^{p}$ estimates for the kinetic solutions, thus providing a proof of uniqueness in the general $L^{1}$ setting. Recall that in this case and in contrast to [16], the kinetic measure is not finite and we only have a weak control of its decay for large $\xi$; cf. Definition 2.1(ii). Moreover, the corresponding chain rule can only be formulated in a weaker sense. In addition, we only assume that $\sigma$ is locally Hölder continuous and $b^{\prime}$ is locally bounded. In particular, no polynomial growth condition for $b^{\prime}$ is required. This generalizes previous related uniqueness results given in [16], Section 3. The additional difficulties are resolved here by introducing an additional cutoff argument chosen adapted to the weaker decay condition (1.6).

Analogously to [16], Proposition 3.1, [32], Proposition 10 and [18], Proposition 3.1, one may prove the existence of left- and right-continuous representatives for kinetic solutions.

Proposition 4.1. Let $u$ be a kinetic solution to (1.1). Then $f=1_{u>\xi}$ admits representatives $f^{-}$and $f^{+}$which are almost surely left and right continuous, respectively, at all points $t^{*} \in[0, T]$ in the sense of distributions over $\mathbb{T}^{N} \times \mathbb{R}$. More precisely, for all $t^{*} \in[0, T]$ there exist kinetic functions $f^{*, \pm}$ on $\Omega \times \mathbb{T}^{N} \times \mathbb{R}$ such that setting $f^{ \pm}\left(t^{*}\right)=f^{*, \pm}$ yields $f^{ \pm}=f$ almost everywhere and

$$
\left\langle f^{ \pm}\left(t^{*} \pm \varepsilon\right), \psi\right\rangle \rightarrow\left\langle f^{ \pm}\left(t^{*}\right), \psi\right\rangle \varepsilon \downarrow 0 \quad \forall \psi \in C_{c}^{2}\left(\mathbb{T}^{N} \times \mathbb{R}\right), \mathbb{P} \text {-a.s. },
$$

where the zero set does not depend on $\psi$ nor $t^{*}$. Moreover, there is a countable set $Q \subseteq[0, T]$ such that $\mathbb{P}$-a.s. for all $t^{*} \in[0, T] \backslash Q$ we have $f^{+}\left(t^{*}\right)=f^{-}\left(t^{*}\right)$.

The next step relies on the doubling of the variables technique. However, it is necessary to establish a new version, which is suitable for the $L^{1}$-setting. 
Let $\left(\varrho_{\varepsilon}\right),\left(\psi_{\delta}\right)$ be standard Dirac sequences on $\mathbb{T}^{N}$ and $\mathbb{R}$, respectively, and let $\left(K_{\ell}\right)$ be a sequence of smooth cut-off functions defined as follows: Let $K \in$ $C^{\infty}(\mathbb{R})$ be such that $0 \leq K(\xi) \leq 1, K \equiv 1$ if $|\xi| \leq 1, K \equiv 0$ if $|\xi| \geq 2$, and $\left|K^{\prime}(\xi)\right| \leq 1$. Define $K_{\ell}(\xi):=K\left(\frac{\xi}{2^{\ell}}\right), \ell \in \mathbb{N}$. Then $\left|K_{\ell}^{\prime}(\xi)\right| \leq \frac{1}{2^{\ell}} \mathbf{1}_{2^{\ell}} \leq|\xi| \leq 2^{\ell+1}$.

In the sequel, we use the following convention: When no integral bounds are specified, we integrate with respect to $(x, y, \xi, \zeta, \eta) \in\left(\mathbb{T}^{N}\right)^{2} \times \mathbb{R}^{3}$. In addition, we only specify the kinetic and Young measures, but omit the Lebesgue measure (also with respect to the time variable).

PROPOSITION 4.2 (Doubling of variables). Let $u_{1}, u_{2}$ be kinetic solutions to (1.1). Denote $f_{1}=\mathbf{1}_{u_{1}>\xi}, f_{2}=\mathbf{1}_{u_{2}>\xi}$ with the corresponding Young measures $v^{1}=\delta_{u^{1}}, v^{2}=\delta_{u^{2}}$, respectively. Then for all $t \in[0, T]$ we have

$$
\begin{aligned}
& \mathbb{E} \int \varrho_{\varepsilon}(x-y) K_{\ell}(\eta) \psi_{\delta}(\eta-\xi) \psi_{\delta}(\eta-\zeta) f_{1}^{ \pm}(x, t, \xi) \bar{f}_{2}^{ \pm}(y, t, \zeta) \\
& \leq \mathbb{E} \int \varrho_{\varepsilon}(x-y) K_{\ell}(\eta) \psi_{\delta}(\eta-\xi) \psi_{\delta}(\eta-\zeta) f_{1,0}(x, \xi) \bar{f}_{2,0}(y, \zeta) \\
& \quad+\mathrm{I}+\mathrm{J}+\mathrm{K}+L(\delta, \ell),
\end{aligned}
$$

where $\lim _{\ell \rightarrow \infty} \lim _{\delta \rightarrow 0} L(\delta, \ell)=0$ and

$$
\begin{aligned}
\mathrm{I}= & \mathbb{E} \int_{0}^{t} \int f_{1} \bar{f}_{2}(b(\xi)-b(\zeta)) \cdot \nabla_{x} \varrho_{\varepsilon}(x-y) K_{\ell}(\eta) \psi_{\delta}(\eta-\xi) \psi_{\delta}(\eta-\zeta), \\
\mathrm{J}= & \mathbb{E} \int_{0}^{t} \int f_{1} \bar{f}_{2}(A(\xi)+A(\zeta)): \mathrm{D}_{x}^{2} \varrho_{\varepsilon}(x-y) K_{\ell}(\eta) \psi_{\delta}(\eta-\xi) \psi_{\delta}(\eta-\zeta) \\
& -\mathbb{E} \int_{0}^{t} \int \varrho_{\varepsilon}(x-y) K_{\ell}(\eta) \psi_{\delta}(\eta-\xi) \mathrm{d} v_{x, s}^{1}(\xi) \mathrm{d} n_{2}^{\psi_{\delta}(\eta-\cdot)}(y, s) \\
& -\mathbb{E} \int_{0}^{t} \int_{\left(\mathbb{T}^{N}\right)^{2}} \int_{\mathbb{R}^{3}} \varrho_{\varepsilon}(x-y) K_{\ell}(\eta) \psi_{\delta}(\eta-\zeta) \mathrm{d} v_{y, s}^{2}(\zeta) \mathrm{d} n_{1}^{\psi_{\delta}(\eta-\cdot)}(x, s), \\
\mathrm{K}= & \frac{1}{2} \mathbb{E} \int_{0}^{t} \int \varrho_{\varepsilon}(x-y) K_{\ell}(\eta) \psi_{\delta}(\eta-\xi) \psi_{\delta}(\eta-\zeta) \\
& \times \sum_{k \geq 1}\left|g_{k}(x, \xi)-g_{k}(y, \zeta)\right|^{2} \mathrm{~d} v_{x, s}^{1}(\xi) \mathrm{d} v_{y, s}^{2}(\zeta) .
\end{aligned}
$$

Proof. A similar approach as in [32], Proposition 3.2, and [19], Proposition 9 , yields for $\alpha(x, \xi, y, \zeta, \eta):=\varrho_{\varepsilon}(x-y) K_{\ell}(\eta) \psi_{\delta}(\eta-\xi) \psi_{\delta}(\eta-\zeta)$ that

$$
\begin{aligned}
& \mathbb{E} \int f_{1}^{+}(t) \bar{f}_{2}^{+}(t) \alpha \\
& =\mathbb{E} \int f_{1,0} \bar{f}_{2,0} \alpha+\mathbb{E} \int_{0}^{t} \int f_{1} \bar{f}_{2}(b(\xi)-b(\zeta)) \cdot \nabla_{x} \alpha \\
& \quad+\mathbb{E} \int_{0}^{t} \int f_{1} \bar{f}_{2} A(\zeta): \mathrm{D}_{y}^{2} \alpha+\mathbb{E} \int_{0}^{t} \int f_{1} \bar{f}_{2} A(\xi): \mathrm{D}_{x}^{2} \alpha
\end{aligned}
$$




$$
\begin{aligned}
& +\frac{1}{2} \mathbb{E} \int_{0}^{t} \int \bar{f}_{2} \partial_{\xi} \alpha G_{1}^{2} \mathrm{~d} v_{x, s}^{1}(\xi)-\frac{1}{2} \mathbb{E} \int_{0}^{t} \int f_{1} \partial_{\zeta} \alpha G_{2}^{2} \mathrm{~d} v_{y, s}^{2}(\zeta) \\
& -\mathbb{E} \int_{0}^{t} \int G_{1,2} \alpha \mathrm{d} v_{x, s}^{1}(\xi) \mathrm{d} v_{y, s}^{2}(\zeta)-\mathbb{E} \int_{0}^{t} \int \bar{f}_{2}^{-} \partial_{\xi} \alpha \mathrm{d} m_{1}(x, s, \xi) \\
& +\mathbb{E} \int_{0}^{t} \int f_{1}^{+} \partial_{\zeta} \alpha \mathrm{d} m_{2}(y, s, \zeta)=: I_{1}+\cdots+I_{9} .
\end{aligned}
$$

We need to treat the last five terms $I_{5}, \ldots, I_{9}$. Using the fact that

$$
\begin{aligned}
\int_{\mathbb{R}} \psi_{\delta} & (\eta-\xi) \partial_{\zeta} \psi_{\delta}(\eta-\zeta) K_{\ell}(\eta) \mathrm{d} \eta \\
& =-\int_{\mathbb{R}} \partial_{\xi} \psi_{\delta}(\eta-\xi) \psi_{\delta}(\eta-\zeta) \mathrm{d} \eta+\int_{\mathbb{R}} \psi_{\delta}(\eta-\xi) \psi_{\delta}(\eta-\zeta) K_{\ell}^{\prime}(\eta) \mathrm{d} \eta
\end{aligned}
$$

together with the decomposition of the kinetic measure $m_{2}$ from Definition 2.2(iii), it follows

$$
\begin{aligned}
I_{9} \leq & -\mathbb{E} \int_{0}^{t} \int \varrho_{\varepsilon}(x-y) K_{\ell}(\eta) \psi_{\delta}(\eta-\xi) \mathrm{d} \nu_{x, s}^{1}(\xi) \mathrm{d} n_{2}^{\psi_{\delta}(\eta-\cdot)}(y, s) \\
& +\frac{1}{2^{\ell}} \mathbb{E} \int_{0}^{t} \int_{\mathbb{T}^{N}} \int_{\mathbb{R}} \mathbf{1}_{2^{\ell}-\delta \leq|\zeta| \leq 2^{\ell+1}+\delta} \mathrm{d} m_{2}(y, s, \zeta),
\end{aligned}
$$

where, according to Definition 2.1(ii), the second term on the right-hand side vanishes if we let $\delta \rightarrow 0$ and then $\ell \rightarrow \infty$. By symmetry,

$$
\begin{aligned}
I_{8} \leq & -\mathbb{E} \int_{0}^{t} \int \varrho_{\varepsilon}(x-y) K_{\ell}(\eta) \psi_{\delta}(\eta-\zeta) \mathrm{d} v_{y, s}^{2}(\zeta) \mathrm{d} n_{1}^{\psi_{\delta}(\eta-\cdot)}(x, s) \\
& +\frac{1}{2^{\ell}} \mathbb{E} \int_{0}^{t} \int_{\mathbb{T}^{N}} \int_{\mathbb{R}} \mathbf{1}_{2^{\ell}-\delta \leq|\xi| \leq 2^{\ell+1}+\delta} \mathrm{d} m_{1}(x, s, \xi) .
\end{aligned}
$$

Next, we have

$$
\begin{aligned}
I_{5}+I_{6} & +I_{7} \\
= & K+\frac{1}{2} \mathbb{E} \int_{0}^{t} \int \bar{f}_{2} \varrho_{\varepsilon}(x-y) \psi_{\delta}(\eta-\xi) \psi_{\delta}(\eta-\zeta) K_{\ell}^{\prime}(\eta) G_{1}^{2} \mathrm{~d} v_{s, x}^{1}(\xi) \\
& -\frac{1}{2} \mathbb{E} \int_{0}^{t} \int f_{1} \varrho_{\varepsilon}(x-y) \psi_{\delta}(\eta-\xi) \psi_{\delta}(\eta-\zeta) K_{\ell}^{\prime}(\eta) G_{2}^{2} \mathrm{~d} \nu_{y, s}^{2}(\zeta) \\
= & : K+I_{51}+I_{61}
\end{aligned}
$$

and due to (2.4)

$$
\begin{aligned}
I_{51} \leq & \frac{D}{2^{\ell}} \mathbb{E} \int_{0}^{t} \int_{\mathbb{T}^{N}} \int_{\mathbb{R}} \mathbf{1}_{2^{\ell}-\delta \leq|\xi| \leq 2^{\ell+1}+\delta} \mathrm{d} v_{s, x}^{1}(\xi) \mathrm{d} x \mathrm{~d} s \\
& +\frac{D}{2^{\ell}} \mathbb{E} \int_{0}^{t} \int_{\mathbb{T}^{N}} \int_{\mathbb{R}} \mathbf{1}_{2^{\ell}-\delta \leq|\xi| \leq 2^{\ell+1}+\delta}|\xi|^{2} \mathrm{~d} \nu_{s, x}^{1}(\xi) \mathrm{d} x \mathrm{~d} s .
\end{aligned}
$$


We further note that

$$
\begin{aligned}
& \frac{D}{2^{\ell}} \mathbb{E} \int_{0}^{t} \int_{\mathbb{T}^{N}} \int_{\mathbb{R}} \mathbf{1}_{2^{\ell}-\delta \leq|\xi| \leq 2^{\ell+1}+\delta}|\xi|^{2} \mathrm{~d} v_{s, x}^{1}(\xi) \mathrm{d} x \mathrm{~d} s \\
& \quad \leq D \frac{2^{\ell+1}+\delta}{2^{\ell}} \mathbb{E} \int_{0}^{t} \int_{\mathbb{T}^{N}} \int_{\mathbb{R}^{\prime}} \mathbf{1}_{2^{\ell}-\delta \leq|\xi| \leq 2^{\ell+1}+\delta}|\xi| \mathrm{d} \nu_{s, x}^{1}(\xi) \mathrm{d} x \mathrm{~d} s \\
& \quad \leq D \frac{2^{\ell+1}+\delta}{2^{\ell}} \mathbb{E} \int_{0}^{t} \int_{\mathbb{T}^{N}} \mathbf{1}_{2^{\ell}-\delta \leq\left|u^{1}(s, x)\right|}\left|u^{1}(s, x)\right| \mathrm{d} x \mathrm{~d} s .
\end{aligned}
$$

Hence,

$$
\limsup _{\delta \rightarrow 0} I_{51} \leq \frac{D}{2^{\ell}} t+D \mathbb{E} \int_{0}^{t} \int_{\mathbb{T}^{N}} \mathbf{1}_{2^{\ell} \leq\left|u^{1}(s, x)\right|}\left|u^{1}(s, x)\right| \mathrm{d} x \mathrm{~d} s .
$$

By dominated convergence, this implies that $\lim _{\ell \rightarrow \infty} \lim _{\delta \rightarrow 0} I_{51}=0$ and $I_{61}$ may be treated analogously, which completes the proof.

Finally, we have all in hand to prove the comparison principle leading to the proof of uniqueness as well as continuous dependence on the initial condition.

THEOREM 4.3 (Comparison principle). Let $u$ be a kinetic solution to (1.1). Then there exist $u^{+}$and $u^{-}$, representatives of $u$, such that, for all $t \in[0, T]$, $f^{ \pm}(t, x, \xi)=\mathbf{1}_{u^{ \pm}(t, x)>\xi}$ for a.e. $(\omega, x, \xi)$, where $f^{ \pm}$are as in Proposition 4.1.

Moreover, if $u_{1}, u_{2}$ are kinetic solutions to (1.1) with initial data $u_{1,0}, u_{2,0}$, respectively, then

$$
\sup _{t \in[0, T]} \mathbb{E}\left\|\left(u_{1}^{ \pm}(t)-u_{2}^{ \pm}(t)\right)^{+}\right\|_{L^{1}\left(\mathbb{T}^{N}\right)} \leq \mathbb{E}\left\|\left(u_{1,0}-u_{2,0}\right)^{+}\right\|_{L^{1}\left(\mathbb{T}^{N}\right)} .
$$

PROOF. Using Proposition 4.2, we have

$$
\begin{aligned}
\mathbb{E} \int_{\mathbb{T}^{N}} & \int_{\mathbb{R}} f_{1}^{ \pm}(x, t, \xi) \bar{f}_{2}^{ \pm}(x, t, \xi) \mathrm{d} \xi \mathrm{d} x \\
= & \mathbb{E} \int \varrho_{\varepsilon}(x-y) K_{\ell}(\eta) \psi_{\delta}(\eta-\xi) \psi_{\delta}(\eta-\zeta) f_{1}^{ \pm}(x, t, \xi) \bar{f}_{2}^{ \pm}(y, t, \zeta) \\
& \quad+\eta_{t}(\varepsilon, \delta, \ell) \\
\leq & \mathbb{E} \int \varrho_{\varepsilon}(x-y) K_{\ell}(\eta) \psi_{\delta}(\eta-\xi) \psi_{\delta}(\eta-\zeta) f_{1,0}(x, \xi) \bar{f}_{2,0}(y, \zeta) \\
& \quad+\mathrm{I}+\mathrm{J}+\mathrm{K}+L(\delta, \ell)+\eta_{t}(\varepsilon, \delta, \ell),
\end{aligned}
$$

with I, J, K as in Proposition 4.2 and $\lim _{\ell \rightarrow \infty} \lim _{\varepsilon, \delta \rightarrow 0} \eta_{t}(\varepsilon, \delta, \ell)=0$. We aim to find suitable bounds for the terms I, J, K. 
Since $b^{\prime}$ is locally bounded, setting $\left\|b^{\prime}\right\|_{\infty, \delta, \ell}:=\left\|b^{\prime}\right\|_{L^{\infty}\left(-2^{\ell+1}-\delta, 2^{\ell+1}+\delta\right)}$, we have

$$
\begin{aligned}
|\mathrm{I}| & \leq\left\|b^{\prime}\right\|_{\infty, \delta, \ell} \mathbb{E} \int_{0}^{t} \int f_{1} \bar{f}_{2}|\xi-\zeta| \psi_{\delta}(\eta-\zeta) \psi_{\delta}(\eta-\xi) K_{\ell}(\eta)\left|\nabla_{x} \varrho_{\varepsilon}(x-y)\right| \\
& \leq 82^{\ell}\left|\mathbb{T}^{N}\right|^{2} \varepsilon^{-1} \delta\left\|b^{\prime}\right\|_{\infty, \delta, \ell} t .
\end{aligned}
$$

In order to estimate the term $\mathrm{J}$, we observe that

$$
\begin{aligned}
\mathrm{J}= & \mathbb{E} \int_{0}^{t} \int f_{1} \bar{f}_{2}(\sigma(\xi)-\sigma(\zeta))^{2}: \mathrm{D}_{x}^{2} \varrho_{\varepsilon}(x-y) K_{\ell}(\eta) \psi_{\delta}(\eta-\xi) \psi_{\delta}(\eta-\zeta) \\
& +2 \mathbb{E} \int_{0}^{t} \int f_{1} \bar{f}_{2} \sigma(\xi) \sigma(\zeta): \mathrm{D}_{x}^{2} \varrho_{\varepsilon}(x-y) K_{\ell}(\eta) \psi_{\delta}(\eta-\xi) \psi_{\delta}(\eta-\zeta) \\
& -\mathbb{E} \int_{0}^{t} \int \varrho_{\varepsilon}(x-y) K_{\ell}(\eta) \psi_{\delta}(\eta-\xi) \mathrm{d} \nu_{x, s}^{1}(\xi) \mathrm{d} n_{2}^{\psi_{\delta}(\eta-\cdot)}(y, s) \\
& -\mathbb{E} \int_{0}^{t} \int \varrho_{\varepsilon}(x-y) K_{\ell}(\eta) \psi_{\delta}(\eta-\zeta) \mathrm{d} v_{y, s}^{2}(\zeta) \mathrm{d} n_{1}^{\psi_{\delta}(\eta-\cdot)}(x, s) \\
= & \mathbf{J}_{1}+\mathrm{J}_{2}+\mathbf{J}_{3}+\mathbf{J}_{4} .
\end{aligned}
$$

Since $\sigma$ is locally $\gamma$-Hölder continuous due to (2.2), it holds

$$
\left|\mathbf{J}_{1}\right| \leq C t \delta^{2 \gamma} \varepsilon^{-2}\|\sigma\|_{C}\left(\left[-2^{\ell+1}-\delta, 2^{\ell+1}+\delta\right]\right) .
$$

Next, we will show that

$$
\mathrm{J}_{2}+\mathrm{J}_{3}+\mathrm{J}_{4} \leq 0
$$

From (2.7) and (2.8), we have

$$
\begin{aligned}
\mathbf{J}_{3}+\mathrm{J}_{4} & \\
= & \mathbb{E} \int_{0}^{t} \int_{\left(\mathbb{T}^{N}\right)^{2}} \int_{\mathbb{R}} \varrho_{\varepsilon}(x-y) K_{\ell}(\eta) \psi_{\delta}\left(\eta-u_{1}\right) \\
& \times\left|\operatorname{div}_{y} \int_{0}^{u_{2}} \sqrt{\psi_{\delta}(\eta-\zeta)} \sigma(\zeta) \mathrm{d} \zeta\right|^{2} \\
& -\mathbb{E} \int_{0}^{t} \int_{\left(\mathbb{T}^{N}\right)^{2}} \int_{\mathbb{R}} \varrho_{\varepsilon}(x-y) K_{\ell}(\eta) \psi_{\delta}\left(\eta-u_{2}\right) \\
& \times\left|\operatorname{div}_{x} \int_{0}^{u_{1}} \sqrt{\psi_{\delta}(\eta-\xi)} \sigma(\xi) \mathrm{d} \xi\right|^{2} \\
\leq & -2 \mathbb{E} \int_{0}^{t} \int_{\left(\mathbb{T}^{N}\right)^{2}} \int_{\mathbb{R}} \varrho_{\varepsilon}(x-y) K_{\ell}(\eta) \sqrt{\psi_{\delta}\left(\eta-u_{1}\right)} \sqrt{\psi_{\delta}}\left(\eta-u_{2}\right) \\
& \times \operatorname{div}_{x} \int_{0}^{u_{1}} \sqrt{\psi_{\delta}(\eta-\xi) \sigma(\xi) \cdot \operatorname{div} y} \int_{0}^{u_{2}} \sqrt{\psi_{\delta}(\eta-\zeta)} \sigma(\zeta)
\end{aligned}
$$




$$
\begin{aligned}
= & -2 \mathbb{E} \int_{0}^{t} \int_{\left(\mathbb{T}^{N}\right)^{2}} \int_{\mathbb{R}} \varrho_{\varepsilon}(x-y) K_{\ell}(\eta) \\
& \times \operatorname{div}_{x} \int_{0}^{u_{1}} \psi_{\delta}(\eta-\xi) \sigma(\xi) \cdot \operatorname{div}_{y} \int_{0}^{u_{2}} \psi_{\delta}(\eta-\zeta) \sigma(\zeta) .
\end{aligned}
$$

Equation (4.2) now follows from

$$
\begin{aligned}
\mathbf{J}_{2}= & 2 \mathbb{E} \int_{0}^{t} \int_{\left(\mathbb{T}^{N}\right)^{2}} \int_{\mathbb{R}} \varrho_{\varepsilon}(x-y) K_{\ell}(\eta) \\
& \times \operatorname{div}_{y} \int_{0}^{u_{2}} \sigma(\zeta) \psi_{\delta}(\eta-\zeta) \mathrm{d} \zeta \cdot \operatorname{div}_{x} \int_{0}^{u_{1}} \sigma(\xi) \psi_{\delta}(\eta-\xi) \leq-J_{3}-J_{4} .
\end{aligned}
$$

Due to (2.6), we have

$$
\begin{aligned}
\mathrm{K} \leq & C \mathbb{E} \int_{0}^{t} \int \varrho_{\varepsilon}(x-y)|x-y|^{2} \psi_{\delta}(\eta-\zeta) \psi_{\delta}(\eta-\xi) K_{\ell}(\eta) \mathrm{d} v_{x, s}^{1}(\xi) \mathrm{d} v_{y, s}^{2}(\zeta) \\
& +C \mathbb{E} \int_{0}^{t} \int \varrho_{\varepsilon}(x-y) \psi_{\delta}(\eta-\zeta) \psi_{\delta}(\eta-\xi) K_{\ell}(\eta)|\xi-\zeta|^{2} \mathrm{~d} v_{x, s}^{1}(\xi) \mathrm{d} \nu_{y, s}^{2}(\zeta) \\
\leq & C t \delta^{-1} \varepsilon^{2}+C t \delta .
\end{aligned}
$$

As a consequence, we deduce, for all $t \in[0, T]$,

$$
\begin{aligned}
\mathbb{E} \int_{\mathbb{T}^{N}} \int_{\mathbb{R}} f_{1}^{ \pm}(x, t, \xi) \bar{f}_{2}^{ \pm}(x, t, \xi) \mathrm{d} \xi \mathrm{d} x \\
\leq \mathbb{E} \int \varrho_{\varepsilon}(x-y) K_{\ell}(\eta) \psi_{\delta}(\eta-\xi) \psi_{\delta}(\eta-\zeta) f_{1,0}(x, \xi) \bar{f}_{2,0}(y, \zeta) \\
\quad+C \varepsilon^{-1} \delta 2^{\ell}\left\|b^{\prime}\right\|_{\infty, \delta, \ell} t+C t \delta^{2 \gamma} \varepsilon^{-2}\|\sigma\|_{C \gamma\left(\left[-2^{\ell+1}-\delta, 2^{\ell+1}+\delta\right]\right)}+C t \delta^{-1} \varepsilon^{2} \\
\quad+C t \delta+L(\delta, \ell)+\eta_{t}(\varepsilon, \delta, \ell) .
\end{aligned}
$$

Taking $\delta=\varepsilon^{\beta}$ with $\beta \in(1 / \gamma, 2)$ and letting $\varepsilon \rightarrow 0$ yields

$$
\begin{aligned}
\mathbb{E} \int_{\mathbb{T}^{N}} & \int_{\mathbb{R}} f_{1}^{ \pm}(x, t, \xi) \bar{f}_{2}^{ \pm}(x, t, \xi) \mathrm{d} \xi \mathrm{d} x \\
\leq & \mathbb{E} \int_{\mathbb{T}^{N}} \int_{\mathbb{R}} K_{\ell}(\eta) f_{1,0}(x, \eta) \bar{f}_{2,0}(x, \eta) \mathrm{d} x \mathrm{~d} \eta \\
& \quad+\lim _{\delta \rightarrow 0} L\left(\varepsilon^{\beta}, \ell\right)+\lim _{\varepsilon \rightarrow 0} \eta_{t}\left(\varepsilon, \varepsilon^{\beta}, \ell\right) .
\end{aligned}
$$

Taking $\ell \rightarrow \infty$, we conclude

$$
\mathbb{E} \int_{\mathbb{T}^{N}} \int_{\mathbb{R}} f_{1}^{ \pm}(x, t, \xi) \bar{f}_{2}^{ \pm}(x, t, \xi) \mathrm{d} \xi \mathrm{d} x \leq \mathbb{E} \int_{\mathbb{T}^{N}} \int_{\mathbb{R}} f_{1,0}(x, \eta) \bar{f}_{2,0}(x, \eta) \mathrm{d} x \mathrm{~d} \eta .
$$

The conclusion of (4.1) from this proceeds along the lines of the proof of [16], Theorem 3.3, where we refer for the details. 
4.2. Existence. In this section, we prove the existence of kinetic solutions. The proof of existence is based on a two-level approximation procedure. One of which is a vanishing viscosity approximation. One difficulty in the construction of a kinetic solution to (1.1) is the verification of the chain-rule in Definition 2.2(ii). In contrast to the other conditions, the chain-rule is not necessarily preserved under taking weak limits, so that strong convergence of the approximating solutions is needed. This strong convergence is particularly hard to obtain in the vanishing viscosity approximation. We resolve this obstacle by employing the regularity estimates established in Section 3.

As mentioned above, the proof of existence proceeds in two steps, corresponding to two layers of approximation. In the first step, we replace the initial condition $u_{0}$ by a smooth, bounded approximation $u_{0}^{\kappa} \in L^{\infty}\left(\Omega \times \mathbb{T}^{N}\right)$ such that $u_{0}^{\kappa} \in C_{c}^{\infty}\left(\mathbb{T}^{N}\right) \mathbb{P}$-a.s. and

$$
u_{0}^{\kappa} \rightarrow u_{0} \quad \text { in } L^{1}\left(\Omega ; L^{1}\left(\mathbb{T}^{N}\right)\right)
$$

and we also replace the diffusion matrix $A$ by a symmetric, positive definite matrix $A^{\kappa}$ given by

$$
A^{\kappa}(\xi):=\kappa \operatorname{Id}+A(\xi), \quad \xi \in \mathbb{R} .
$$

In the second step, we replace $A^{\kappa}$ by a bounded, symmetric and positive definite matrix $A^{\kappa, \tau}$ given by its square root

$$
\sigma_{i j}^{\kappa, \tau}(\xi)=\sqrt{\kappa} \delta_{i j}+ \begin{cases}\sigma_{i j}(\xi) & \text { if }|\xi| \leq \frac{1}{\tau} \\ \sigma_{i j}\left(\frac{\operatorname{sgn}(\xi)}{\tau}\right) & \text { if }|\xi|>\frac{1}{\tau}\end{cases}
$$

and we further approximate the flux $B$ by $B^{\tau}$, defined by setting

$$
\left(b^{\tau}\right)^{\prime}(\xi):= \begin{cases}b^{\prime}(\xi) & \text { if }|\xi| \leq \frac{1}{\tau} \\ b^{\prime}\left(\frac{\operatorname{sgn}(\xi)}{\tau}\right) & \text { if }|\xi|>\frac{1}{\tau} .\end{cases}
$$

Since $\sigma$ and $b^{\prime}$ are locally bounded, $\sigma^{\kappa, \tau}$ and $\left(b^{\tau}\right)^{\prime}$ are bounded for each $\kappa, \tau>0$ fixed and $B^{\tau}$ is of sub-quadratic growth. Moreover,

$$
A^{\kappa, \tau}(\xi)=\kappa \operatorname{Id}+A(\xi), \quad B^{\tau}(\xi)=B(\xi) \quad \forall|\xi| \leq \frac{1}{\tau},
$$

and thus $A^{\kappa, \tau} \rightarrow A$ locally uniformly. Hence, we consider

$$
\begin{aligned}
\mathrm{d} u^{\kappa, \tau}+\operatorname{div}\left(B^{\tau}\left(u^{\kappa, \tau}\right)\right) \mathrm{d} t & =\operatorname{div}\left(A^{\kappa, \tau}\left(u^{\kappa, \tau}\right) \nabla u^{\kappa, \tau}\right) \mathrm{d} t+\Phi\left(u^{\kappa, \tau}\right) \mathrm{d} W, \\
u^{\kappa, \tau}(0) & =u_{0}^{\kappa} .
\end{aligned}
$$

In the case of (4.3), the results from [16], Section 4, are applicable, which yields the existence and uniqueness of a weak solution to (4.3) and appropriate bounds. 
We will then pass to the limit, first employing the compactness method from [32], Section 4.3 , for the limit $\tau \rightarrow 0$, then proving the strong convergence in $L^{1}$ as $\kappa \rightarrow 0$ directly using the regularity properties established in Section 3.

In the following subsection, we establish uniform $L^{p}$ bounds on the approximating solutions. Next, in Section 4.2.2 we prove uniform bounds on the corresponding kinetic dissipation measures and we conclude the proof of existence in Section 4.2.3.

4.2.1. $L^{p}$-estimates. Let us start with an a priori $L^{p}$-estimate for solutions to (1.1).

Proposition 4.4. Let $u$ be a kinetic solution to (1.1). Then

$$
\underset{0 \leq t \leq T}{\mathbb{E} \operatorname{ess} \sup }\|u(t)\|_{L^{p}}^{p q} \leq C_{T, p, q}\left(1+\mathbb{E}\left\|u_{0}\right\|_{L^{p}}^{p q}\right)
$$

for all $p, q \in[1, \infty)$ and for some constant $C_{T, p, q}>0$.

PROOF. The proof relies on the Itô formula applied to (1.1) and the function

$$
u \mapsto \int_{\mathbb{T}^{N}}\left(1+|u|^{2}\right)^{\frac{p}{2}} \mathrm{~d} x=\left\|\left(1+|u|^{2}\right)^{\frac{p}{4}}\right\|_{L^{2}}^{2} .
$$

In order to make the following calculations rigorous, one works on the level of the approximations $u^{\kappa, \tau}$ introduced above. This leads to a uniform estimate which implies (4.4) for the (unique) limiting kinetic solution $u$ by lower-semicontinuity of the norm. Since this limiting procedure is standard, we restrict to presenting the main, informal arguments here.

Itô's formula yields

$$
\begin{aligned}
\left\|\left(1+|u(t)|^{2}\right)^{\frac{p}{4}}\right\|_{L^{2}}^{2}= & \left\|\left(1+\left|u_{0}\right|^{2}\right)^{\frac{p}{4}}\right\|_{L^{2}}^{2} \\
& -p \int_{0}^{t} \int_{\mathbb{T}^{N}}\left(1+|u|^{2}\right)^{\frac{p}{2}-1} u \operatorname{div}(B(u)) \mathrm{d} x \mathrm{~d} s \\
& +p \int_{0}^{t} \int_{\mathbb{T}^{N}}\left(1+|u|^{2}\right)^{\frac{p}{2}-1} u \operatorname{div}(A(u) \nabla u) \mathrm{d} x \mathrm{~d} s \\
& +p \sum_{k \geq 1} \int_{0}^{t} \int_{\mathbb{T}^{N}}\left(1+|u|^{2}\right)^{\frac{p}{2}-1} u g_{k}(x, u) \mathrm{d} x \mathrm{~d} \beta_{k} \\
& +\frac{p}{2} \int_{0}^{t} \int_{\mathbb{T}^{N}}\left(1+|u|^{2}\right)^{\frac{p}{2}-2}\left(1+(p-1)|u|^{2}\right) G^{2}(x, u) \mathrm{d} x \mathrm{~d} s .
\end{aligned}
$$

Due to the periodic boundary conditions, the second term on the right-hand side vanishes after an integration by parts. The third term is nonpositive due to integration by parts and positive semidefinitness of $A$. The fourth one may be estimated, 
using (2.5), by

$$
\begin{aligned}
& \left|\frac{p}{2} \int_{0}^{t} \int_{\mathbb{T}^{N}}\left(1+|u|^{2}\right)^{\frac{p}{2}-2}\left(1+(p-1)|u|^{2}\right) G^{2}(x, u) \mathrm{d} x \mathrm{~d} s\right| \\
& \quad \leq C \int_{0}^{t} \int_{\mathbb{T}^{N}}\left(1+|u|^{2}\right)^{\frac{p}{2}} \mathrm{~d} x \mathrm{~d} s=C \int_{0}^{t}\left\|\left(1+|u(s)|^{2}\right)^{\frac{p}{4}}\right\|_{L^{2}}^{2} \mathrm{~d} s .
\end{aligned}
$$

Finally, for the stochastic integral, taking the supremum, the $q^{\text {th }}$ power and the expectation, we have by Burkholder-Davis-Gundy's inequality, (2.5) and Young's inequality

$$
\begin{aligned}
\mathbb{E} \sup _{0 \leq t \leq T}\left|p \sum_{k \geq 1} \int_{0}^{t} \int_{\mathbb{T}^{N}}\left(1+|u|^{2}\right)^{\frac{p}{2}-1} u g_{k}(x, u) \mathrm{d} x \mathrm{~d} \beta_{k}\right|^{q} \\
\leq C \mathbb{E}\left(\int_{0}^{T}\left\|\left(1+|u|^{2}\right)^{\frac{p}{4}}\right\|_{L^{2}}^{2} \sum_{k \geq 1}\left\|\left(1+|u|^{2}\right)^{\frac{p}{4}-\frac{1}{2}}\left|g_{k}(u)\right|\right\|_{L^{2}}^{2} \mathrm{~d} t\right)^{\frac{q}{2}} \\
\quad \leq \frac{1}{2} \sup _{0 \leq t \leq T}\left\|\left(1+|u|^{2}\right)^{\frac{p}{4}}\right\|_{L^{2}}^{2 q}+C_{T} \mathbb{E} \int_{0}^{T}\left\|\left(1+|u|^{2}\right)^{\frac{p}{4}}\right\|_{L^{2}}^{2 q} \mathrm{~d} t .
\end{aligned}
$$

Thus, Gronwall's lemma yields

$$
\mathbb{E} \sup _{0 \leq t \leq T}\left\|\left(1+|u|^{2}\right)^{\frac{p}{4}}\right\|_{L^{2}}^{2 q} \leq C_{T, q}\left\|\left(1+\left|u_{0}\right|^{2}\right)^{\frac{p}{4}}\right\|_{L^{2}}^{2 q} .
$$

Since

$$
\|u\|_{L^{p}}^{p q} \leq\left\|\left(1+|u|^{2}\right)^{\frac{p}{4}}\right\|_{L^{2}}^{2 q} \leq C_{p, q}\left(1+\|u\|_{L^{p}}^{p q}\right),
$$

this concludes the proof.

4.2.2. Decay of the kinetic measure. To appreciate the difficulty and methods introduced in the following, we recall that in the deterministic case

$$
\partial_{t} f(t)+b(\xi) \cdot \nabla f(t)+A(\xi): \mathrm{D}^{2} f(t)=\partial_{\xi} m
$$

bounds on the kinetic measure $m$ are easily derived (informally) by testing with $1_{[k, \infty)}(\xi)$ and integrating in $t, x, \xi$, which corresponds to computing the derivative $\partial_{t}(u-k)_{+}$via the chain-rule. In the stochastic case, this has to be replaced by the Itô formula informally leading to terms of the form $\int g_{k}^{2}(x, u) \delta_{u=k} \mathrm{~d} \xi \mathrm{d} x \mathrm{~d} t$ which are not easy to control. Therefore, new techniques are needed in the stochastic case and a less restrictive decay assumption on the kinetic measure is used [cf. the discussion before (1.6)].

LEMMA 4.5. Let $u_{0} \in L^{r}\left(\Omega ; L^{1}\left(\mathbb{T}^{N}\right)\right)$ for some $r \in[1, \infty)$ and let $u$ be a kinetic solution to (1.1). Then, for all $k \in \mathbb{N}$,

$$
\mathbb{E}\left|m\left([0, T] \times \mathbb{T}^{N} \times[-k, k]\right)\right|^{r} \leq C\left(r, k, T, \mathbb{E}\left\|u_{0}\right\|_{L^{1}}^{r}\right),
$$

for some $C>0$ depending on $D$ only. 
Proof. Step 1: For $k>0$, set

$$
\begin{aligned}
\theta_{k}(u) & =\mathbf{1}_{[-k, k]}(u), \\
\Theta_{k}(u) & =\int_{-k}^{u} \int_{-k}^{r} \theta_{k}(s) \mathrm{d} s \mathrm{~d} r .
\end{aligned}
$$

Let $\gamma \in C_{c}^{1}([0, T))$ be nonnegative such that $\gamma(0)=1, \gamma^{\prime} \leq 0$. Then after a preliminary step of regularization we may take $\varphi(t, x, \xi)=\gamma(t) \Theta_{k}^{\prime}(\xi)$ in (2.9) to get

$$
\begin{aligned}
\mathbb{E}\left|\int_{0}^{T} \int_{\mathbb{T}^{N}} \Theta_{k}(u(t, x))\right| \gamma^{\prime}(t)|\mathrm{d} x \mathrm{~d} t|^{r}+\mathbb{E}\left|\int_{A_{k}^{+}} \gamma(t) \mathrm{d} m(t, x, \xi)\right|^{r} \\
\leq \mathbb{E}\left|\int_{\mathbb{T}^{N}} \Theta_{k}\left(u_{0}(x)\right) \mathrm{d} x\right|^{r} \\
\quad+\mathbb{E}\left|\int_{0}^{T} \int_{\mathbb{T}^{N}} \gamma(t) G^{2}(x, u(t, x)) \theta_{k}(u(t, x)) \mathrm{d} x \mathrm{~d} t\right|^{r} \\
\quad+\mathbb{E}\left|\sum_{j \geq 1} \int_{0}^{T} \int_{\mathbb{T}^{N}} g_{j}(x, u(t, x)) \Theta_{k}^{\prime}(u(t, x)) \gamma(t) \mathrm{d} x \mathrm{~d} \beta_{k}(t)\right|^{r},
\end{aligned}
$$

where $A_{k}^{+}=[0, T] \times \mathbb{T}^{N} \times[-k, k]$. Since $0 \leq \Theta_{k}(u) \leq 2 k(k+|u|)$ and, due to (2.5),

$$
\frac{1}{2} G^{2}(x, u) \theta_{k}(u) \leq \frac{1}{2} D(1+|u|)^{2} \mathbf{1}_{[-k, k]}(u) \leq D\left(1+k^{2}\right) .
$$

Since $0 \leq \Theta_{k}^{\prime}(u) \leq 2 k \mathbf{1}_{-k \leq u}$, we may estimate the stochastic integral using the Burkholder-Davis-Gundy inequality, the Minkowski integral inequality, (2.5) and the Young inequality as follows:

$$
\begin{aligned}
\mathbb{E} \mid \sum_{j \geq 1} & \left.\int_{0}^{T} \int_{\mathbb{T}^{N}} g_{j}(x, u(t, x)) \Theta_{k}^{\prime}(u(t, x)) \gamma(t) \mathrm{d} x \mathrm{~d} \beta_{j}(t)\right|^{r} \\
& \lesssim \mathbb{E}\left(\int_{0}^{T} \sum_{j \geq 1}\left(\int_{\mathbb{T}^{N}} g_{j}(x, u) \Theta_{k}^{\prime}(u) \mathrm{d} x\right)^{2} \mathrm{~d} t\right)^{\frac{r}{2}} \\
& \leq 2 k^{r} \mathbb{E}\left(\int_{0}^{T}\left(\int_{\mathbb{T}^{N}}\left(\sum_{j \geq 1}\left|g_{j}(x, u)\right|^{2}\right)^{\frac{1}{2}} \mathrm{~d} x\right)^{2} \mathrm{~d} t\right)^{\frac{r}{2}} \\
& \leq 2 D k^{r} \mathbb{E}\left(\int_{0}^{T}\left(\int_{\mathbb{T}^{N}}(1+|u|) \mathrm{d} x\right)^{2} \mathrm{~d} t\right)^{\frac{r}{2}} \\
& \leq D k^{r} \mathbb{E} \sup _{0 \leq t \leq T}\|1+u\|_{L^{1}}^{r}+D k^{r} \mathbb{E}\left(\int_{0}^{T}\|1+u\|_{L^{1}} \mathrm{~d} t\right)^{r} \\
& \leq C\left(D, k^{r}, T^{r}, \mathbb{E}\left\|u_{0}\right\|_{L^{1}}^{r}\right),
\end{aligned}
$$


where we also used Proposition 4.4 with $p=1, q=r$ for the last step. The claim follows.

LEMMA 4.6. Let $u_{0} \in L^{1}\left(\Omega ; L^{1}\left(\mathbb{T}^{N}\right)\right)$, $u$ be a kinetic solution to (1.1) and $\Theta \in C^{2}(\mathbb{R})$ be nonnegative, convex such that $\Theta^{\prime \prime}(\xi)\left(1+|\xi|^{2}\right) \leq C_{\Theta}(1+\Theta(\xi))$ for some constant $C_{\Theta}>0$. Then

$$
\begin{aligned}
\sup _{t \in[0, T]} & \mathbb{E} \int_{\mathbb{T}^{N}} \Theta(u(t, x)) \mathrm{d} x+\mathbb{E} \int_{0}^{T} \int \Theta^{\prime \prime}(\xi) \mathrm{d} m(t, x, \xi) \\
\leq & C\left(\mathbb{E} \int_{\mathbb{T}^{N}} \Theta\left(u_{0}(x)\right) \mathrm{d} x+1\right)
\end{aligned}
$$

for some $C>0$ depending on $D, C_{\Theta}$ only.

Proof. Let $\Theta$ be as in the statement and $\gamma \in C_{c}^{1}([0, T))$ be nonnegative such that $\gamma(0)=1, \gamma^{\prime} \leq 0$. Taking $\varphi(t, x, \xi)=\gamma(t) \Theta^{\prime}(\xi)$ in (2.9) we get

$$
\begin{aligned}
\mathbb{E} \int_{0}^{T} \int_{\mathbb{T}^{N}} \Theta(u(t, x))\left|\gamma^{\prime}(t)\right| \mathrm{d} x \mathrm{~d} t+\mathbb{E} \int \Theta^{\prime \prime}(\xi) \gamma(t) \mathrm{d} m(t, x, \xi) \\
\quad \lesssim \mathbb{E} \int_{0}^{T} \int_{\mathbb{T}^{N}} \gamma(t) G^{2}(x, u(t, x)) \Theta^{\prime \prime}(u(t, x)) \mathrm{d} x \mathrm{~d} t \\
\quad+\mathbb{E} \int_{\mathbb{T}^{N}} \Theta\left(u_{0}(x)\right) \mathrm{d} x .
\end{aligned}
$$

By assumption,

$$
\frac{1}{2} G^{2}(x, u) \Theta^{\prime \prime}(u) \leq D\left(1+|u|^{2}\right) \Theta^{\prime \prime}(u) \leq C(1+\Theta(u)) .
$$

Letting now $\gamma \rightarrow 1_{[0, t]}$, an application of Gronwall's lemma completes the proof.

We proceed with an estimate which is a modification of [17], Proposition 16, and applies to the case multiplicative noise.

Proposition 4.7. Let $u_{0} \in L^{1}\left(\Omega ; L^{1}\left(\mathbb{T}^{N}\right)\right)$ and let $u$ be a kinetic solution to (1.1). Then

$$
\begin{aligned}
& \underset{0 \leq t \leq T}{\operatorname{ess} \sup } \mathbb{E}\left\|\left(u(t)-2^{n}\right)^{+}\right\|_{L_{x}^{1}}+\underset{0 \leq t \leq T}{\operatorname{ess} \sup } \mathbb{E}\left\|\left(u(t)+2^{n}\right)^{-}\right\|_{L_{x}^{1}}+\frac{1}{2^{n}} \mathbb{E} m\left(A_{2^{n}}\right) \\
& \leq C\left(T, \mathbb{E}\left\|u_{0}\right\|_{L_{x}^{1}}\right) \delta(n) \quad \forall n \in \mathbb{N}_{0},
\end{aligned}
$$

where

$$
A_{2^{n}}=[0, T] \times \mathbb{T}^{N} \times\left\{\xi \in \mathbb{R} ; 2^{n} \leq|\xi| \leq 2^{n+1}\right\}
$$


and $\delta(n)$ depends only on $D$ and on the functions

$$
R \mapsto \mathbb{E}\left\|\left(u_{0}-R\right)^{+}\right\|_{L_{x}^{1}}, \quad R \mapsto \mathbb{E}\left\|\left(u_{0}+R\right)^{-}\right\|_{L_{x}^{1}},
$$

and satisfies $\lim _{n \rightarrow \infty} \delta(n)=0$.

Furthermore,

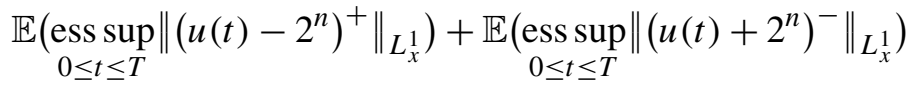

$$
\begin{aligned}
& \leq C\left(T, D, \mathbb{E}\left\|u_{0}\right\|_{L_{x}^{1}}\right)\left[\delta(n)+\delta^{\frac{1}{2}}(n)\right],
\end{aligned}
$$

where $\delta(n)$ is as above, in addition possibly depending on the function

$$
R \mapsto \mathbb{E}\left\|(1+|u|) 1_{R \leq|u|}\right\|_{L_{x, t}^{1}}
$$

ProOF. Step 1: For $k>0$, set

$$
\theta_{k}(u)=\frac{1}{k} \mathbf{1}_{k \leq u \leq 2 k}, \quad \Theta_{k}(u)=\int_{0}^{u} \int_{0}^{r} \theta_{k}(s) \mathrm{d} s \mathrm{~d} r .
$$

Let $\eta=(21 D) \vee 1, \gamma \in C_{c}^{1}([0, \eta T))$ be nonnegative such that $\gamma(0)=1, \gamma^{\prime} \leq 0$. Then taking $\varphi(t, x, \xi)=\gamma(\eta t) \Theta_{k}^{\prime}(\xi)$ in (2.9) we get

$$
\begin{aligned}
\eta \mathbb{E} \int_{0}^{T} & \int_{\mathbb{T}^{N}} \Theta_{k}(u(t, x))\left|\gamma^{\prime}(\eta t)\right| \mathrm{d} x \mathrm{~d} t+\frac{1}{k} \mathbb{E} \int_{A_{k}^{+}} \gamma(\eta t) \mathrm{d} m(t, x, \xi) \\
= & \frac{1}{2} \mathbb{E} \int_{0}^{T} \int_{\mathbb{T}^{N}} \gamma(\eta t) G^{2}(x, u(t, x)) \theta_{k}(u(t, x)) \mathrm{d} x \mathrm{~d} t \\
& +\mathbb{E} \int_{\mathbb{T}^{N}} \Theta_{k}\left(u_{0}(x)\right) \mathrm{d} x,
\end{aligned}
$$

where $A_{k}^{+}=[0, T] \times \mathbb{T}^{N} \times\{\xi \in \mathbb{R} ; k \leq \xi \leq 2 k\}$. Note that

$$
\left(u-\frac{3}{2} k\right)^{+} \leq \Theta_{k}(u) \leq(u-k)^{+},
$$

and, using (2.5),

$$
\frac{1}{2 \eta} G^{2}(x, u) \theta_{k}(u) \leq D \frac{1+|u|^{2}}{\eta k} \mathbf{1}_{k \leq u \leq 2 k} \leq D \frac{1+4 k^{2}}{\eta k} \frac{(u-l)^{+}}{k-l}
$$

for all $k>l \geq 0, u \in \mathbb{R}$. We choose $l=\frac{3}{4} k$ and observe, by choice of $\eta$ and for $k \geq 1$,

$$
\alpha:=D \frac{1+4 k^{2}}{\eta k(k-l)}=4 D \frac{1+4 k^{2}}{\eta k^{2}}<1 .
$$


Consequently, we deduce from (4.7) that

$$
\begin{aligned}
& \mathbb{E} \int_{0}^{T} \int_{\mathbb{T}^{N}}(u(t, x)-2 l)^{+}\left|\gamma^{\prime}(\eta t)\right| \mathrm{d} x \mathrm{~d} t \\
& \leq \alpha \mathbb{E} \int_{0}^{T} \int_{\mathbb{T}^{N}}(u(t, x)-l)^{+} \gamma(\eta t) \mathrm{d} x \mathrm{~d} t \\
& \quad+\mathbb{E} \int_{\mathbb{T}^{N}}\left(u_{0}(x)-l\right)^{+} \mathrm{d} x
\end{aligned}
$$

We can now iterate the above procedure by replacing $k$ by $2 k$. To do so, we need to check that applying (4.7) to $\tilde{k}=2 k$, the chosen constant $\alpha$ appearing in (4.10) is the same as before. The condition (4.9) now reads $4 D \frac{1+4(2 k)^{2}}{4 \eta k^{2}}<1$, which holds true due to (4.9). We deduce that

$$
\begin{aligned}
& \mathbb{E} \int_{0}^{T} \int_{\mathbb{T}^{N}}(u(t, x)-4 l)^{+}\left|\gamma^{\prime}(\eta t)\right| \mathrm{d} x \mathrm{~d} t \\
& \quad \leq \alpha \mathbb{E} \int_{0}^{T} \int_{\mathbb{T}^{N}}(u(t, x)-2 l)^{+} \gamma(\eta t) \mathrm{d} x \mathrm{~d} t+\mathbb{E} \int_{\mathbb{T}^{N}}\left(u_{0}(x)-2 l\right)^{+} \mathrm{d} x .
\end{aligned}
$$

Since the same argument can be applied to $\tilde{k}=2^{n} k, \tilde{l}=2^{n} l$ for any $n \in \mathbb{N}$, we set

$$
\psi_{n}(t):=\mathbb{E} \int_{\mathbb{T}^{N}}\left(u(t, x)-2^{n} l\right)^{+} \mathrm{d} x
$$

and after letting $\gamma$ approximate $\mathbf{1}_{[0, \eta t]}$ we finally obtain

$$
\psi_{n+1}(t) \leq \alpha \int_{0}^{t} \psi_{n}(s) \mathrm{d} s+\psi_{n}(0)
$$

Now we proceed similarly as in [17], Proposition 16. Since $(u-l)^{+} \leq(u-2 l)^{+}+$ $l$, we have

$$
\begin{aligned}
\psi_{0}(t) & =\mathbb{E} \int_{\mathbb{T}^{N}}(u(t, x)-l)^{+} \mathrm{d} x \\
& \leq \mathbb{E} \int_{\mathbb{T}^{N}}(u(t, x)-2 l)^{+} \mathrm{d} x+l \\
& =\psi_{1}(t)+l
\end{aligned}
$$

so

$$
\psi_{1}(t) \leq \alpha \int_{0}^{t} \psi_{0}(s) \mathrm{d} s+\psi_{0}(0) \leq \alpha \int_{0}^{t} \psi_{1}(s) \mathrm{d} s+\alpha l t+\psi_{0}(0)
$$

and Gronwall's lemma implies

$$
\psi_{0}(t) \leq M:=C\left(T,\left\|u_{0}\right\|_{L^{1}}\right) .
$$


Thus, we deduce

$$
\psi_{1}(t) \leq \alpha t M+\psi_{0}(0), \quad \psi_{2}(t) \leq \alpha^{2} \frac{t^{2}}{2} M+\alpha t \psi_{0}(0)+\psi_{1}(0),
$$

and generally

$$
\psi_{n+1}(t) \leq \alpha^{n+1} \frac{t^{n+1}}{n+1 !} M+\sum_{k=0}^{n} \alpha^{k} \frac{t^{k}}{k !} \psi_{n-k}(0)
$$

Let

$$
\delta(n)=\alpha^{n}+\sum_{k=0}^{n-1} \alpha^{k} \psi_{n-1-k}(0)
$$

which satisfies $\delta(n) \rightarrow 0$ as $n \rightarrow \infty$ due to the assumption on $u_{0}$. Therefore, it follows that

$$
\psi_{n+1}(t) \leq(M+1) \mathrm{e}^{T} \delta(n+1)
$$

and consequently,

$$
\underset{0 \leq t \leq T}{\operatorname{ess} \sup } \mathbb{E} \int_{\mathbb{T}^{N}}\left(u(t, x)-2^{n+1} l\right)^{+} \mathrm{d} x \leq C\left(T, \mathbb{E}\left\|u_{0}\right\|_{L_{x}^{1}}\right) \delta(n+1) .
$$

Thus, as a consequence of (4.7), (4.8) and the fact that $k>l$ we get

$$
\begin{aligned}
& \underset{0 \leq t \leq T}{\operatorname{ess} \sup } \mathbb{E} \int_{\mathbb{T}^{N}}\left(u(t, x)-2^{n} k\right)^{+} \mathrm{d} x+\frac{1}{2^{n} k \eta} \mathbb{E} \int_{A_{2^{n} k}^{+}} \mathrm{d} m(t, x, \xi) \\
& \leq C\left(T, \mathbb{E}\left\|u_{0}\right\|_{L_{x}^{1}}\right) \delta(n) .
\end{aligned}
$$

Choosing $k=1$ completes the proof.

Step 2: To prove (4.6), we start similarly as in Step 1 but take the supremum in time before taking the expectation. The iterative inequality then reads

$$
\begin{aligned}
& \underset{0 \leq t \leq T}{\mathbb{E} \operatorname{ess} \sup } \int_{\mathbb{T}^{N}}\left(u(t, x)-2^{n+1} l\right)^{+} \mathrm{d} x \mathrm{~d} t \\
& \leq \alpha \mathbb{E} \int_{0}^{T} \int_{\mathbb{T}^{N}}\left(u(t, x)-2^{n} l\right)^{+} \mathrm{d} x \mathrm{~d} t+\mathbb{E} \int_{\mathbb{T}^{N}}\left(u_{0}(x)-2^{n} l\right)^{+} \mathrm{d} x \\
& \quad+\mathbb{E} \sup _{0 \leq t \leq T}\left|\sum_{j \geq 1} \int_{0}^{t} \int_{\mathbb{T}^{N}} g_{j}(x, u(t, x)) \Theta_{2^{n} k}^{\prime}(u(t, x)) \mathrm{d} x \mathrm{~d} \beta_{j}(t)\right| \\
& \leq C\left(T, \alpha, \mathbb{E}\left\|u_{0}\right\|_{L_{x}^{1}}\right) \delta(n) \\
&+C \mathbb{E}\left(\int_{0}^{T} \int_{\mathbb{T}^{N}} G^{2}(x, u(t, x))\left|\Theta_{2^{n} k}^{\prime}(u(t, x))\right|^{2} \mathrm{~d} x \mathrm{~d} t\right)^{\frac{1}{2}} .
\end{aligned}
$$


Using (2.5), we estimate

$$
\begin{aligned}
\mathbb{E} \sup _{0 \leq t \leq T}\left|\sum_{j \geq 1} \int_{0}^{t} \int_{\mathbb{T}^{N}} g_{j}(x, u(t, x)) \Theta_{2^{n} k}^{\prime}(u(t, x)) \mathrm{d} x \mathrm{~d} \beta_{j}(t)\right| \\
\quad \lesssim \mathbb{E}\left(\int_{0}^{T}\left(\int_{\mathbb{T}^{N}}(1+|u|) \mathbf{1}_{2^{n} k \leq u} \mathrm{~d} x\right)^{2} \mathrm{~d} t\right)^{\frac{1}{2}} \\
\quad \lesssim\left(\mathbb{E} \sup _{0 \leq t \leq T}\|1+|u|\|_{L_{x}^{1}}\right)^{\frac{1}{2}}\left(\mathbb{E} \int_{0}^{T} \int_{\mathbb{T}^{N}}(1+|u|) \mathbf{1}_{2^{n} k \leq u} \mathrm{~d} x \mathrm{~d} t\right)^{\frac{1}{2}} .
\end{aligned}
$$

The right-hand side converges to 0 as $n \rightarrow \infty$ due to the dominated convergence theorem. Hence the estimate (4.6) follows using Proposition 4.4.

Based on the equi-integrability estimate (4.6), we can deduce that kinetic solutions have continuous paths in $L^{1}\left(\mathbb{T}^{N}\right)$ a.s.

Corollary 4.8 (Continuity in time). Let $u_{0} \in L^{1}\left(\Omega ; L^{1}\left(\mathbb{T}^{N}\right)\right)$ and let $u$ be a kinetic solution to (1.1). Then there exists a representative of $u$ with almost surely continuous trajectories in $L^{1}\left(\mathbb{T}^{N}\right)$.

PROOF. Based on Proposition 4.1, Theorem 4.3 and (4.6) we are in a position to apply [17], Lemma 17, which implies the continuity in $L^{1}$. Indeed, let us first show that $u^{+}$constructed in Theorem 4.3 is $\mathbb{P}$-a.s. right continuous in $L^{1}\left(\mathbb{T}^{N}\right)$. Due to Proposition 4.1, we have that $f^{+}(t+\varepsilon) \rightarrow^{*} f^{+}(t)$ in $L^{\infty}\left(\mathbb{T}^{N} \times \mathbb{R}\right) \mathbb{P}_{-}$ a.s. as $\varepsilon \rightarrow 0$. Due to Theorem 4.3, for all $t \in[0, T)$ the kinetic function $f^{+}(t)$ is at equilibrium, that is, $f^{+}(t, x, \xi)=\mathbf{1}_{u^{+}(t, x)>\xi}$ for a.e. $(\omega, x, \xi)$. Finally, (4.6) implies

$$
\lim _{n \rightarrow \infty} \mathbb{E} \sup _{t \in[0, T]}\left\|\left(u^{+}(t)-2^{n}\right)^{ \pm}\right\|_{L_{x}^{1}}=0
$$

Hence, there exists a subsequence (not relabeled) which converges $\mathbb{P}$-a.s., that is, P-a.s.,

$$
\lim _{n \rightarrow \infty} \sup _{t \in[0, T]}\left\|\left(u^{+}(t)-2^{n}\right)^{ \pm}\right\|_{L_{x}^{1}}=0 .
$$

Consequently, [17], Lemma 17, applies and yields the convergence

$$
u^{+}(t+\varepsilon) \rightarrow u^{+}(t) \quad \text { in } L^{1}\left(\mathbb{T}^{N}\right) \text { as } \varepsilon \rightarrow 0 .
$$

The same arguments show that $u^{-}$constructed in Theorem 4.3 is $\mathbb{P}$-a.s. left continuous in $L^{1}\left(\mathbb{T}^{N}\right)$. Finally, the fact that $u^{+}(t)=u^{-}(t)$ for all $t \in[0, T]$ can be proved as in [19], Corollary 12. 


\subsubsection{The proof of existence.}

THEOREM 4.9. Let $u_{0} \in L^{r}\left(\Omega ; L^{1}\left(\mathbb{T}^{N}\right)\right)$ for some $r>1$ and assume (2.5). There exists a kinetic solution $u$ to $(1.1)$ such that $u \in C\left([0, T] ; L^{1}\left(\mathbb{T}^{N}\right)\right), \mathbb{P}$-a.s. and for all $p, q \in[1, \infty)$ there exists a constant $C>0$ such that

$$
\mathbb{E} \underset{t \in[0, T]}{\operatorname{ess} \sup }\|u(t)\|_{L^{p}}^{p q} \leq C\left(1+\mathbb{E}\left\|u_{0}\right\|_{L^{p}}^{p q}\right)
$$

Proof. Step 1: According to [16], Section 4, there exists a unique kinetic solution $u^{\kappa, \tau}$ to (4.3) with uniform $L^{p}$-bounds by [16], Theorem 5.2. The construction of a kinetic solution $\left(u^{\kappa}, m^{\kappa}\right)$ to (1.1) with $A$ replaced by $A^{\kappa}$ then follows along the same lines as [32], Section 4.3, Section 4.5, where we refer for the details. In this case, the parabolic dissipation defines a nonnegative measure $n_{1}^{\kappa}$ given in [16], Definition 2.2.

Step 2: First, we observe that the assumption (2.3) is satisfied uniformly in $\kappa$. Indeed, let

$$
\mathcal{L}^{\kappa}(i u, i n, \xi):=i(u+b(\xi) \cdot n)+n^{*} A^{\kappa}(\xi) n
$$

Then, for some constant $C>0$,

$$
\left\{\xi \in \mathbb{R} ; \mid \mathcal{L}^{\kappa}(\text { iu, in }, \xi) \mid \leq \delta\right\} \subset\{\xi \in \mathbb{R} ; \mid \mathcal{L}(\text { iu }, \text { in }, \xi) \mid \leq C \delta\}
$$

which implies for all $\phi \in C_{c}^{\infty}(\mathbb{R})$

$$
\omega_{\mathcal{L}^{\kappa}}^{\phi}(J ; \delta) \leq \omega_{\mathcal{L}}^{\phi}(J ; C \delta) \lesssim_{\phi}\left(\frac{\delta}{J^{\beta}}\right)^{\alpha} \quad \forall \delta>0, \forall J \gtrsim 1 .
$$

Moreover, $\mathcal{L}_{\xi}^{\kappa}(i u, i n, \xi)=\mathcal{L}_{\xi}(i u, i n, \xi)$, and thus

$$
\sup _{\substack{u \in \mathbb{R}, n \in \mathbb{Z}^{N} \\|n| \sim J}} \sup _{\xi \in \operatorname{supp} \eta}\left|\mathcal{L}_{\xi}^{\kappa}(i u, i n, \xi)\right| \leq \sup _{\substack{u \in \mathbb{R}, n \in \mathbb{Z}^{N} \\|n| \sim J}} \sup _{\xi \in \operatorname{supp} \eta}\left|\mathcal{L}_{\xi}(i u, i n, \xi)\right| \lesssim_{\phi} J^{\beta}
$$

Consequently, Theorem 3.1 applies and we obtain, with $\left(u^{\kappa}\right)^{\phi}:=\int_{\mathbb{R}} \chi_{u^{\kappa}} \phi \mathrm{d} \xi$,

$$
\begin{aligned}
& \left(\mathbb{E} \int_{0}^{T}\left\|\left(u^{\kappa}\right)^{\phi}(t)\right\|_{\left.W_{x}^{s, r} \mathrm{~d} t\right)^{1 / r}}^{r}\right. \\
& \quad \lesssim_{\phi}\left\|u_{0}^{\kappa}\right\|_{L_{\omega, x}^{1}}^{1 / 2}+\left\|u^{\kappa}\right\|_{L_{\omega, t, x}^{1}}^{1 / 2}+\sup _{0 \leq t \leq T}\left\|u^{\kappa}(t)\right\|_{L_{\omega, x}^{1}}+\left\|m^{\kappa} \mathbf{1}_{\operatorname{supp} \phi}\right\|_{L_{\omega}^{1} \mathcal{M}_{t, x, \xi}}+1
\end{aligned}
$$

for some $r>1$ and $s>0$. In view of Proposition 4.4 and Lemma 4.5, the righthand side can be further estimated uniformly in $\kappa$ as follows:

$$
\left(\mathbb{E} \int_{0}^{T}\left\|\left(u^{\kappa}\right)^{\phi}(t)\right\|_{W_{x}^{s, r}}^{r} \mathrm{~d} t\right)^{1 / r} \lesssim_{\phi}\left\|u_{0}\right\|_{L_{\omega, x}^{1}}+1
$$


Step 3: We next prove that $\left(u^{\kappa}\right)$ is Cauchy in $L^{1}(\Omega \times[0, T], \mathcal{P}, \mathrm{dP} \otimes$ $\left.\mathrm{d} t ; L^{1}\left(\mathbb{T}^{N}\right)\right)$. This is based on computations similar to Section 4.1. Accordingly, let $\left(\varrho_{\varepsilon}\right),\left(\psi_{\delta}\right)$ and $\left(K_{\ell}\right)$ be as in Section 4.1 and let us use the same integral convention.

For any two approximate solutions $u^{\kappa_{1}}, u^{\kappa_{2}}$, we have

$$
\begin{aligned}
\mathbb{E} \int_{\mathbb{T}^{N}} & \left(u^{\kappa_{1}}(t)-u^{\kappa_{2}}(t)\right)^{+} \mathrm{d} x \\
= & \mathbb{E} \int_{\mathbb{T}^{N}} \int_{\mathbb{R}} f^{\kappa_{1}}(x, t, \xi) \bar{f}^{\kappa_{2}}(x, t, \xi) \mathrm{d} \xi \mathrm{d} x \\
= & \mathbb{E} \int f^{\kappa_{1}}(x, t, \xi) \bar{f}^{\kappa_{2}}(y, t, \zeta) K_{\ell}(\eta) \varrho_{\varepsilon}(x-y) \psi_{\delta}(\eta-\zeta) \psi_{\delta}(\eta-\xi) \\
& +\eta_{t}\left(\kappa_{1}, \kappa_{2}, \varepsilon, \delta, \ell\right),
\end{aligned}
$$

where $\varepsilon, \delta$ and $\ell$ are chosen arbitrarily and their value will be fixed later. The idea now is to show that the mollification error $\eta_{t}\left(\kappa_{1}, \kappa_{2}, \varepsilon, \delta, \ell\right)$ can be made arbitrarily small uniformly in $\kappa_{1}, \kappa_{2}$, which will rely on the equi-integrability estimate, Proposition 4.7, as well as (4.11) based on the averaging lemma, Theorem 3.1, the a priori $L^{p}$-estimates, Proposition 4.4 and the bound for the kinetic measure from Lemma 4.5. Indeed, we write

$$
\begin{aligned}
& \eta_{t}\left(\kappa_{1}, \kappa_{2}, \varepsilon, \delta, \ell\right) \\
& =\mathbb{E} \int_{\mathbb{T}^{N}} \int_{\mathbb{R}} f^{\kappa_{1}}(x, t, \eta) \bar{f}^{\kappa_{2}}(x, t, \eta) \mathrm{d} \eta \mathrm{d} x \\
& -\mathbb{E} \int f^{\kappa_{1}}(x, t, \xi) \bar{f}^{\kappa_{2}}(y, t, \zeta) K_{\ell}(\eta) \varrho_{\varepsilon}(x-y) \psi_{\delta}(\eta-\zeta) \psi_{\delta}(\eta-\xi) \\
& =\mathbb{E} \int_{\mathbb{T}^{N}} \int_{\mathbb{R}} f^{\kappa_{1}}(x, t, \eta) \bar{f}^{\kappa_{2}}(x, t, \eta)\left(1-K_{\ell}(\eta)\right) \mathrm{d} \eta \mathrm{d} x \\
& +\left(\mathbb{E} \int_{\mathbb{T}^{N}} \int_{\mathbb{R}} f^{\kappa_{1}}(x, t, \eta) \bar{f}^{\kappa_{2}}(x, t, \eta) K_{\ell}(\eta) \mathrm{d} \eta \mathrm{d} x\right. \\
& \left.-\mathbb{E} \int_{\left(\mathbb{T}^{N}\right)^{2}} \int_{\mathbb{R}} f^{\kappa_{1}}(x, t, \eta) \bar{f}^{\kappa_{2}}(y, t, \eta) K_{\ell}(\eta) \varrho_{\varepsilon}(x-y) \mathrm{d} \eta \mathrm{d} x \mathrm{~d} y\right) \\
& +\left(\mathbb{E} \int_{\left(\mathbb{T}^{N}\right)^{2}} \int_{\mathbb{R}} f^{\kappa_{1}}(x, t, \eta) \bar{f}^{\kappa_{2}}(y, t, \eta) K_{\ell}(\eta) \varrho_{\varepsilon}(x-y) \mathrm{d} \eta \mathrm{d} x \mathrm{~d} y\right. \\
& \left.-\mathbb{E} \int_{\left(\mathbb{T}^{N}\right)^{2}} \int_{\mathbb{R}^{2}} f^{\kappa_{1}}(x, t, \eta) \bar{f}^{\kappa_{2}}(y, t, \zeta) K_{\ell}(\eta) \varrho_{\varepsilon}(x-y) \psi_{\delta}(\eta-\zeta)\right) \\
& +\left(\mathbb{E} \int_{\left(\mathbb{T}^{N}\right)^{2}} \int_{\mathbb{R}^{2}} f^{\kappa_{1}}(x, t, \eta) \bar{f}^{\kappa_{2}}(y, t, \zeta) K_{\ell}(\eta) \varrho_{\varepsilon}(x-y) \psi_{\delta}(\eta-\zeta)\right. \\
& \left.-\mathbb{E} \int f^{\kappa_{1}}(x, t, \xi) \bar{f}^{\kappa_{2}}(y, t, \zeta) K_{\ell}(\eta) \varrho_{\varepsilon}(x-y) \psi_{\delta}(\eta-\xi) \psi_{\delta}(\eta-\zeta)\right) \\
& =\mathrm{H}_{1}+\mathrm{H}_{2}+\mathrm{H}_{3}+\mathrm{H}_{4}
\end{aligned}
$$


and estimate each of the error terms on the right-hand side separately using the above mentioned results. First,

$$
\begin{aligned}
\left|\mathrm{H}_{1}\right| \leq & \mathbb{E} \int_{\mathbb{T}^{N}} \mathbf{1}_{u^{\kappa_{1}}(t, x)>u^{\kappa_{2}}(t, x)} \int_{u^{\kappa_{2}}(t, x)}^{u^{\kappa_{1}}(t, x)} \mathbf{1}_{|\eta| \geq 2^{\ell}} \mathrm{d} \eta \mathrm{d} x \\
\leq & \mathbb{E} \int_{\mathbb{T}^{N}}\left(u^{\kappa_{1}}(t, x)-2^{\ell}\right)^{+} \mathrm{d} x \\
& +\mathbb{E} \int_{\mathbb{T}^{N}}\left(-2^{\ell}-u^{\kappa_{2}}(t, x)\right)^{+} \mathrm{d} x .
\end{aligned}
$$

Now we observe that the result of Proposition 4.7 holds true for the approximate solutions $u^{\kappa_{1}}, u^{\kappa_{2}}$ uniformly in $\kappa_{1}, \kappa_{2}$. Consequently, $\left|\mathrm{H}_{1}\right| \leq \delta(\ell)$, where $\delta(\ell)$ was defined in Proposition 4.7, and $\lim _{\ell \rightarrow \infty} \sup _{0 \leq t \leq T}\left|\mathrm{H}_{1}\right|=0$ uniformly in $\kappa_{1}, \kappa_{2}$, $\varepsilon, \delta$. Second, in order to estimate

$$
\left|\mathrm{H}_{2}\right|=\left|\mathbb{E} \int_{\left(\mathbb{T}^{N}\right)^{2}} \varrho_{\varepsilon}(x-y) \int_{\mathbb{R}} K_{\ell}(\eta) \mathbf{1}_{u^{\kappa_{1}}(t, x)>\eta}\left[\mathbf{1}_{u^{\kappa_{2}}(t, x) \leq \eta}-\mathbf{1}_{u^{\kappa_{2}}(t, y) \leq \eta}\right]\right|,
$$

we write

$$
\begin{aligned}
\left|\int_{\mathbb{R}} K_{\ell}(\eta) \mathbf{1}_{u^{\kappa_{1}}(x)>\eta}\left[\mathbf{1}_{u^{\kappa_{2}}(x) \leq \eta}-\mathbf{1}_{u^{\kappa_{2}}(y) \leq \eta}\right] \mathrm{d} \eta\right| \\
=\int_{\mathbb{R}} K_{\ell}(\eta) \mathbf{1}_{u^{\kappa_{1}}(t, x)>\eta}\left[\mathbf{1}_{\eta \in\left[u^{\kappa_{2}}(x), u^{\kappa_{2}}(y)\right)}+\mathbf{1}_{\eta \in\left[u^{\kappa_{2}}(y), u^{\left.\kappa_{2}(x)\right)}\right] \mathrm{d} \eta}\right. \\
\leq \mathbf{1}_{u^{\kappa_{2}}(x)<u^{\kappa_{2}}(y)} \int_{\mathbb{R}} K_{\ell}(\eta)\left[\chi_{u^{\kappa_{2}}(x)}(\eta)-\chi_{u^{\kappa_{2}}(y)}(\eta)\right] \mathrm{d} \eta \\
\quad+\mathbf{1}_{u^{\kappa_{2}}(y)<u^{\kappa_{2}}(x)} \int_{\mathbb{R}} K_{\ell}(\eta)\left[\chi_{u^{\kappa_{2}}(y)}(\eta)-\chi_{u^{\kappa_{2}(x)}}(\eta)\right] \mathrm{d} \eta .
\end{aligned}
$$

Thus, using (4.11)

$$
\left|H_{2}\right| \leq \mathbb{E} \int_{\left(\mathbb{T}^{N}\right)^{2}} \varrho_{\varepsilon}(x-y)\left|\left(u^{\kappa_{2}}\right)^{K_{\ell}}(t, x)-\left(u^{\kappa_{2}}\right)^{K_{\ell}}(t, y)\right| \mathrm{d} x \mathrm{~d} y \leq C_{\ell} \varepsilon^{s} .
$$

Hence, for all $\ell \in \mathbb{N}, \lim _{\varepsilon \rightarrow 0} \int_{0}^{T}\left|\mathrm{H}_{2}\right| \mathrm{d} t=0$ uniformly in $\kappa_{1}, \kappa_{2}, \delta$. Third,

$$
\begin{aligned}
\left|\mathrm{H}_{3}\right|= & \mid \mathbb{E} \int_{\left(\mathbb{T}^{N}\right)^{2}} \varrho_{\varepsilon}(x-y) \int_{\mathbb{R}} K_{\ell}(\eta) \mathbf{1}_{u^{\kappa_{1}}(t, x)>\eta} \\
& \times \int_{\mathbb{R}} \psi_{\delta}(\eta-\zeta)\left[\mathbf{1}_{u^{\kappa_{1}}(t, y) \leq \eta}-\mathbf{1}_{\left.u^{\kappa_{1}}(t, y) \leq \zeta\right] \mid} \mid\right. \\
\leq & \frac{1}{2} \mathbb{E} \int_{\left(\mathbb{T}^{N}\right)^{2}} \varrho_{\varepsilon}(x-y) \int_{u^{\kappa} 1(t, y)}^{\min \left\{u^{\kappa_{1}}(t, x), u^{\kappa_{1}}(t, y)+\delta\right\}} \mathrm{d} \eta \mathrm{d} x \mathrm{~d} y \\
& +\frac{1}{2} \mathbb{E} \int_{\left(\mathbb{T}^{N}\right)^{2}} \varrho_{\varepsilon}(x-y) \int_{u^{\kappa_{1}}(t, y)-\delta}^{\min \left\{u^{\kappa_{1}}(t, x), u^{\kappa_{1}}(t, y)\right\}} \mathrm{d} \eta \mathrm{d} x \mathrm{~d} y \leq C \delta
\end{aligned}
$$


hence $\lim _{\delta \rightarrow 0} \sup _{0 \leq t \leq T}\left|\mathrm{H}_{3}\right|=0$ uniformly in $\kappa_{1}, \kappa_{2}, \varepsilon, \ell$. And finally

$$
\begin{aligned}
\left|\mathrm{H}_{4}\right|= & \mid \mathbb{E} \varrho_{\varepsilon}(x-y) \mathbf{1}_{u^{\kappa_{2}}(t, y) \leq \zeta} K_{\ell}(\eta) \psi_{\delta}(\eta-\zeta) \\
& \times\left[\mathbf{1}_{u^{\kappa_{1}}(t, x)>\eta}-\mathbf{1}_{u^{\kappa_{1}}(t, x)>\xi}\right] \psi_{\delta}(\eta-\xi) \mid \\
\leq & \mathbb{E} \int_{\left(\mathbb{T}^{N}\right)^{2}} \varrho_{\varepsilon}(x-y) \int_{\mathbb{R}^{2}} \mathbf{1}_{u^{\kappa_{2}}(t, y) \leq \zeta} \psi_{\delta}(\eta-\zeta) \int_{\eta}^{\eta+\delta} \mathbf{1}_{\eta<u^{\kappa_{1}}(t, x) \leq \xi} \psi_{\delta}(\eta-\xi) \\
& +\mathbb{E} \int_{\left(\mathbb{T}^{N}\right)^{2}} \varrho_{\varepsilon}(x-y) \int_{\mathbb{R}^{2}} \mathbf{1}_{u^{\kappa_{2}}(t, y) \leq \zeta} \psi_{\delta}(\eta-\zeta) \\
& \times \int_{\eta-\delta}^{\eta} \mathbf{1}_{\xi<u^{\kappa_{1}}(t, x) \leq \eta} \psi_{\delta}(\eta-\xi) \\
\leq & \mathbb{E} \int_{\left(\mathbb{T}^{N}\right)^{2}} \varrho_{\varepsilon}(x-y) \int_{u^{\kappa_{2}}(t, y)}^{\infty} \int_{u^{\kappa_{1}} 1(t, x)-\delta}^{u^{\kappa_{1}}(t, x)} \psi_{\delta}(\eta-\zeta) \mathrm{d} \zeta \mathrm{d} \eta \mathrm{d} x \mathrm{~d} y \\
& +\mathbb{E} \int_{\left(\mathbb{T}^{N}\right)^{2}} \varrho_{\varepsilon}(x-y) \int_{u^{\kappa_{2}(t, y)}}^{\infty} \int_{u^{\kappa_{1}}(t, x)}^{u^{\kappa_{1}}(t, x)+\delta} \psi_{\delta}(\eta-\zeta) \mathrm{d} \zeta \mathrm{d} \eta \mathrm{d} x \mathrm{~d} y \leq \delta
\end{aligned}
$$

and, therefore, $\lim _{\delta \rightarrow 0} \sup _{0 \leq t \leq T}\left|\mathrm{H}_{4}\right|=0$ uniformly in $\kappa_{1}, \kappa_{2}, \varepsilon, \ell$. Heading back to (4.12) and using the same calculations as in Proposition 4.2, we deduce that

$$
\begin{gathered}
\mathbb{E} \int_{\mathbb{T}^{N}}\left(u^{\kappa_{1}}(t)-u^{\kappa_{2}}(t)\right)^{+} \mathrm{d} x \leq \eta_{t}\left(\kappa_{1}, \kappa_{2}, \varepsilon, \delta, \ell\right)+\eta_{0}\left(\kappa_{1}, \kappa_{2}, \varepsilon, \delta, \ell\right) \\
+\mathrm{I}+\mathrm{J}+\mathrm{J}^{\#}+\mathrm{K}+L(\delta, \ell),
\end{gathered}
$$

where, with $\delta(\ell)$ as in Proposition 4.7,

$$
\lim _{\ell \rightarrow \infty} \sup _{0 \leq t \leq T} L(\delta, \ell)=\lim _{\ell \rightarrow \infty} \delta(\ell)=0 \quad \text { uniformly in } \kappa_{1}, \kappa_{2}, \varepsilon, \delta .
$$

The terms I, J, K are defined and can be dealt with exactly as in Proposition 4.2 and Theorem 4.3. The term $\mathrm{J}^{\#}$ is defined as

$$
\begin{aligned}
\mathrm{J}^{\#}= & \left(\kappa_{1}+\kappa_{2}\right) \mathbb{E} \int_{0}^{t} \int f^{\kappa_{1}} \bar{f}^{\kappa_{2}} \Delta_{x} \varrho_{\varepsilon}(x-y) K_{\ell}(\eta) \psi_{\delta}(\eta-\xi) \psi_{\delta}(\eta-\zeta) \\
& -\mathbb{E} \int_{0}^{t} \int \varrho_{\varepsilon}(x-y) K_{\ell}(\eta) \psi_{\delta}(\eta-\xi) \psi_{\delta}(\eta-\zeta) \mathrm{d} \nu_{x, s}^{\kappa_{1}}(\xi) \mathrm{d} n_{2}^{\kappa_{2}}(y, s, \zeta) \\
& -\mathbb{E} \int_{0}^{t} \int \varrho_{\varepsilon}(x-y) K_{\ell}(\eta) \psi_{\delta}(\eta-\xi) \psi_{\delta}(\eta-\zeta) \mathrm{d} \nu_{y, s}^{\kappa_{2}}(\zeta) \mathrm{d} n_{2}^{\kappa_{1}}(x, s, \xi),
\end{aligned}
$$

where we used the notation $\nu_{x, s}^{\kappa_{1}}(\xi)=\delta_{u^{\kappa_{1}}(s, x)}(\xi)$ and similarly for $v_{y, s}^{\kappa_{2}}(\zeta)$. Thus,

$$
\begin{aligned}
\mathrm{J}^{\#}= & \left(\kappa_{1}+\kappa_{2}\right) \\
& \times \mathbb{E} \int_{0}^{t} \int_{\left(\mathbb{T}^{N}\right)^{2}} \int_{\mathbb{R}} \varrho_{\varepsilon}(x-y) K_{\ell}(\eta) \psi_{\delta}\left(\eta-u^{\kappa_{1}}\right) \psi_{\delta}\left(\eta-u^{\kappa_{2}}\right) \nabla_{x} u^{\kappa_{1}} \cdot \nabla_{y} u^{\kappa_{2}}
\end{aligned}
$$




$$
\begin{aligned}
& -\kappa_{1} \mathbb{E} \int_{0}^{t} \int_{\left(\mathbb{T}^{N}\right)^{2}} \int_{\mathbb{R}} \varrho_{\varepsilon}(x-y) K_{\ell}(\eta) \psi_{\delta}\left(\eta-u^{\kappa_{1}}\right) \psi_{\delta}\left(\eta-u^{\kappa_{2}}\right)\left|\nabla_{x} u^{\kappa_{1}}\right|^{2} \\
& -\kappa_{2} \mathbb{E} \int_{0}^{t} \int_{\left(\mathbb{T}^{N}\right)^{2}} \int_{\mathbb{R}} \varrho_{\varepsilon}(x-y) K_{\ell}(\eta) \psi_{\delta}\left(\eta-u^{\kappa_{1}}\right) \psi_{\delta}\left(\eta-u^{\kappa_{2}}\right)\left|\nabla_{y} u^{\kappa_{2}}\right|^{2} \\
\leq & \left(\sqrt{\kappa_{1}}-\sqrt{\kappa_{2}}\right)^{2} \\
& \times \mathbb{E} \int_{0}^{t} \int_{\left(\mathbb{T}^{N}\right)^{2}} \int_{\mathbb{R}} \varrho_{\varepsilon}(x-y) K_{\ell}(\eta) \psi_{\delta}\left(\eta-u^{\kappa_{1}}\right) \psi_{\delta}\left(\eta-u^{\kappa_{2}}\right) \nabla_{x} u^{\kappa_{1}} \cdot \nabla_{y} u^{\kappa_{2}} \\
\leq & \left(\sqrt{\kappa_{1}}-\sqrt{\kappa_{2}}\right)^{2} \mathbb{E} \int_{0}^{t} \int f^{\kappa_{1}} \bar{f}^{\kappa_{2}} K_{\ell}(\eta) \psi_{\delta}(\eta-\xi) \psi_{\delta}(\eta-\zeta)\left|\Delta_{x} \varrho_{\varepsilon}(x-y)\right| \\
\leq & C\left(\sqrt{\kappa_{1}}-\sqrt{\kappa_{2}}\right)^{2} \varepsilon^{-2} 2^{\ell},
\end{aligned}
$$

where we proceeded as for I in the last step. Consequently, we see that

$$
\begin{aligned}
& \mathbb{E} \int_{0}^{T} \int_{\mathbb{T}^{N}}\left(u^{\kappa 1}(t)-u^{\kappa_{2}}(t)\right)^{+} \mathrm{d} x \mathrm{~d} t \\
& \lesssim T \\
& \quad \delta(\ell) \\
& \quad+C_{\ell} \varepsilon^{s}+\delta+\varepsilon^{-1} \delta 2^{\ell}\left\|b^{\prime}\right\|_{L^{\infty}(-2 \ell-\delta, 2 \ell+\delta)} t+\delta^{2 \gamma} \varepsilon^{-2}\|\sigma\|_{C}([-\ell-\delta, \ell+\delta]) \\
& \quad+\delta^{-1} \varepsilon^{2}+\delta+\left(\kappa_{1}+\kappa_{2}\right) \varepsilon^{-2} 2^{\ell} .
\end{aligned}
$$

Therefore, given $\vartheta>0$ one can fix $\ell$ sufficiently large so that the first term on the right-hand side is estimated by $\vartheta / 3$, then fix $\varepsilon$ and $\delta$ small enough so that the second and third line is also estimated by $\vartheta / 3$ and then find $\iota>0$ such that the third line is estimated by $\vartheta / 3$ for any $\kappa_{1}, \kappa_{2}<\iota$. Thus, we have shown that the set of approximate solutions $\left(u^{\kappa}\right)$ is Cauchy in $L^{1}\left(\Omega \times[0, T], \mathcal{P}, \mathrm{d} \mathbb{P} \otimes \mathrm{d} t ; L^{1}\left(\mathbb{T}^{N}\right)\right)$, as $\kappa \rightarrow 0$. Hence there exists $u \in L^{1}\left(\Omega \times[0, T], \mathcal{P}, \mathrm{dP} \otimes \mathrm{d} t ; L^{1}\left(\mathbb{T}^{N}\right)\right)$ such that

$$
u^{\kappa} \rightarrow u \quad \text { in } L^{1}\left(\Omega \times[0, T], \mathcal{P}, \mathrm{d} \mathbb{P} \otimes \mathrm{d} t ; L^{1}\left(\mathbb{T}^{N}\right)\right) .
$$

Step 4: Since $u_{0} \in L^{r}\left(\Omega ; L^{1}\left(\mathbb{T}^{N}\right)\right)$ for some $r>1$, we can choose $\left(u_{0}^{\kappa}\right)$ uniformly bounded in $L^{r}\left(\Omega ; L^{1}\left(\mathbb{T}^{N}\right)\right)$. By Lemma 4.5 , we obtain that, for each $k>0$,

$$
\sup _{\kappa} \mathbb{E}\left|m^{\kappa}\left(B_{k}\right)\right|^{r} \leq C_{k},
$$

where $B_{k}:=[0, T] \times \mathbb{T}^{N} \times[-k, k]$. Consequently, the sequence $\left(m^{\kappa}\right)$ is bounded in $L^{r}\left(\Omega ; \mathcal{M}\left(B_{k}\right)\right)$. Following the same arguments as [17], proof of Theorem 20, we extract a subsequence (not relabeled) and a random Borel measure $m$ on $[0, T] \times \mathbb{T}^{N} \times \mathbb{R}$ such that $m^{\kappa} \rightarrow^{*} m$ weakly* in $L^{r}\left(\Omega ; \mathcal{M}\left(B_{k}\right)\right)$ for every $k \in \mathbb{N}$.

Since the estimates derived in Proposition 4.7 are uniform with respect to $\kappa$, the limit $m$ satisfies Definition 2.1(ii). We further note that $m$ satisfies Definition 2.1(i), since this property is stable with respect to weak limits. Hence, $m$ is a kinetic measure. 
We next check that $(u, m)$ is a kinetic solution to (1.1) in the sense of Definition 2.2. Let $\phi \in C_{c}^{\infty}(\mathbb{R})$ be nonnegative and denote by $\Phi$ a function satisfying $\Phi^{\prime \prime}=\phi$ and $\Phi \geq 0$. Then, similar to (4.5), we obtain

$$
\begin{aligned}
& \mathbb{E} \int_{[0, T] \times \mathbb{T}^{N} \times \mathbb{R}} \phi(\xi) \mathrm{d} m^{\kappa}(t, x, \xi) \\
& \quad \leq \frac{1}{2} \mathbb{E} \int_{0}^{T} \int_{\mathbb{T}^{N}} G^{2}\left(x, u^{\kappa}(t, x)\right) \phi\left(u^{\kappa}(t, x)\right) \mathrm{d} x \mathrm{~d} t+\mathbb{E} \int_{\mathbb{T}^{N}} \Phi\left(u_{0}^{\kappa}(x)\right) \mathrm{d} x .
\end{aligned}
$$

Hence, due to (2.5) and since

$$
\begin{aligned}
& \mathbb{E} \int_{[0, T] \times \mathbb{T}^{N} \times \mathbb{R}} \phi(\xi) \mathrm{d} n_{1}^{\kappa}(t, x, \xi) \\
& \quad=\mathbb{E} \int_{0}^{T} \int_{\mathbb{T}^{N}}\left|\operatorname{div} \int_{0}^{u^{\kappa}} \sqrt{\phi(\zeta)}[\sqrt{\kappa} \mathrm{Id}+\sigma(\zeta)] \mathrm{d} \zeta\right|^{2} \mathrm{~d} x \mathrm{~d} t,
\end{aligned}
$$

we obtain that

$$
\begin{aligned}
& \mathbb{E} \int_{0}^{T} \int_{\mathbb{T}^{N}}\left|\operatorname{div} \int_{0}^{u^{\kappa}} \sqrt{\phi(\zeta)}[\sqrt{\kappa} \mathrm{Id}+\sigma(\zeta)] \mathrm{d} \zeta\right|^{2} \mathrm{~d} x \mathrm{~d} t \\
& \leq C\left(T,\|\phi\|_{L^{\infty}}, \operatorname{supp} \phi, \mathbb{E}\left\|u_{0}\right\|_{L_{x}^{1}}\right) .
\end{aligned}
$$

From the strong convergence (4.13) and the fact that $\sqrt{\phi} \sigma \in C_{c}(\mathbb{R})$, we conclude using integration by parts, for all $\eta \in L^{2}\left(0, T ; C^{1}\left(\mathbb{T}^{N}\right)\right), \psi \in L^{\infty}(\Omega)$,

$$
\begin{gathered}
\mathbb{E} \psi \int_{0}^{T} \int_{\mathbb{T}^{N}}\left(\operatorname{div} \int_{0}^{u^{\kappa}} \sqrt{\phi(\zeta)}[\sqrt{\kappa} \mathrm{Id}+\sigma(\zeta)] \mathrm{d} \zeta\right) \eta(t, x) \mathrm{d} x \mathrm{~d} t \\
\rightarrow \mathbb{E} \psi \int_{0}^{T} \int_{\mathbb{T}^{N}}\left(\operatorname{div} \int_{0}^{u} \sqrt{\phi(\zeta)} \sigma(\zeta) \mathrm{d} \zeta\right) \eta(t, x) \mathrm{d} x \mathrm{~d} t
\end{gathered}
$$

and, therefore, using (4.15),

$$
\operatorname{div} \int_{0}^{u^{\kappa}} \sqrt{\phi(\zeta)}[\sqrt{\kappa} \operatorname{Id}+\sigma(\zeta)] \mathrm{d} \zeta \rightarrow \operatorname{div} \int_{0}^{u} \sqrt{\phi(\zeta)} \sigma(\zeta) \mathrm{d} \zeta
$$

in $L^{2}\left(\Omega \times[0, T] \times \mathbb{T}^{N}\right)$. Hence, Definition 2.2(i) is satisfied.

Concerning the chain rule formula (2.7), we observe that the corresponding version holds true for all $u^{\kappa}$, since $u^{\kappa}$ is a kinetic solution, that is, for any $\phi_{1}, \phi_{2} \in$ $C_{c}(\mathbb{R}), \phi_{1}, \phi_{2} \geq 0$,

$$
\begin{aligned}
& \operatorname{div} \int_{0}^{u^{\kappa}} \phi_{1}(\zeta) \phi_{2}(\zeta)[\sqrt{\kappa} \operatorname{Id}+\sigma(\zeta)] \mathrm{d} \zeta \\
& \quad=\phi_{1}\left(u^{\kappa}\right) \operatorname{div} \int_{0}^{u^{\kappa}} \phi_{2}(\zeta)[\sqrt{\kappa} \operatorname{Id}+\sigma(\zeta)] \mathrm{d} \zeta
\end{aligned}
$$

holds true as an equality in $L^{2}\left(\Omega \times[0, T] \times \mathbb{T}^{N}\right)$. Due to (4.16), we can pass to the limit on the left-hand side and, making use of the strong-weak convergence, also on the right-hand side of (4.17). In conclusion, Definition 2.2(ii) holds. 
Let now $\phi \in C_{c}^{\infty}(\mathbb{R}), \phi \geq 0$, and let $n^{\phi}$ be defined as in Definition 2.2(iii). Since $u^{\kappa}$ is a kinetic solution, $\left(u^{\kappa}, m^{\kappa}\right)$ satisfy (2.9) with the corresponding diffusion matrix $A^{\kappa}$. Passing to the limit $\kappa \rightarrow 0$ yields (2.9) for $(u, m)$ with the original diffusion matrix $A$. It remains to prove that, for all $\varphi \in C_{c}^{\infty}\left([0, T] \times \mathbb{T}^{N}\right)$, $\varphi \geq 0, m(\varphi \phi) \geq n^{\phi}(\varphi) \mathbb{P}$-a.s. Since each $m^{\kappa}$ can be decomposed into the sum of the parabolic dissipation measure $n_{1}^{\kappa}$ and the corresponding (nonnegative) entropy dissipation measure $n_{2}^{\kappa}$, that is, $m^{\kappa}=n_{1}^{\kappa}+n_{2}^{\kappa}$, from (4.14) it follows that

$$
\sup _{\kappa} \mathbb{E}\left|n_{1}^{\kappa}\left(B_{k}\right)\right|^{r} \leq C_{k} .
$$

By the same argument as above, we extract a subsequence (not relabeled) and a random measure $o_{1}$ such that $n_{1}^{\kappa} \rightarrow^{*} o_{1}$ weakly* in $L^{r}\left(\Omega ; \mathcal{M}\left(B_{k}\right)\right)$ for all $k \in \mathbb{N}$. Since $m^{\kappa} \geq n_{1}^{\kappa} \mathbb{P}$-a.s., we have $m \geq o_{1}$, $\mathbb{P}$-a.s. Moreover, since by sequentially weak lower semicontinuity of the norm, it follows for all $\varphi \in L^{\infty}\left([0, T] \times \mathbb{T}^{N}\right)$, $\psi \in L^{\infty}(\Omega), \mathbb{P}$-a.s.,

$$
\begin{aligned}
\mathbb{E} \psi & n^{\phi}\left(\varphi^{2}\right) \\
& =\mathbb{E} \psi \int_{0}^{T} \int_{\mathbb{T}^{N}}\left|\operatorname{div} \int_{0}^{u} \sqrt{\phi(\zeta)} \sigma(\zeta) \mathrm{d} \zeta\right|^{2} \varphi^{2}(t, x) \mathrm{d} x \mathrm{~d} t \\
& \leq \liminf _{\kappa \rightarrow 0} \Psi \int_{0}^{T} \int_{\mathbb{T}^{N}}\left|\operatorname{div} \int_{0}^{u^{\kappa}} \sqrt{\phi(\zeta)}[\sqrt{\kappa} \operatorname{Id}+\sigma(\zeta)] \mathrm{d} \zeta\right|^{2} \varphi^{2}(t, x) \mathrm{d} x \mathrm{~d} t \\
& =\mathbb{E} \psi o_{1}\left(\phi \varphi^{2}\right)
\end{aligned}
$$

and thus, $n^{\phi}$ given by (2.8) satisfies $n^{\phi}(\cdot) \leq o_{1}(\phi \cdot), \mathbb{P}$-a.s., which completes the proof.

\section{APPENDIX: MULTIPLIER LEMMAS}

We state a result concerning Fourier multipliers used in Section 3. For the proof, we refer the reader to [51], namely to Lemma 2.2 and the discussion at the end of the proof of averaging Lemma 2.1.

Lemma A.1 (Multiplier lemma). Let $m(u, n, \xi)=i(u+b(\xi) \cdot n)+n^{*} A(\xi) n$ and let $\psi$ be a bump function. For each $\xi \in \mathbb{R}$ and $\delta>0$, let

$$
\Omega(u, n ; \delta):=\left\{\xi \in \mathbb{R} ; \frac{m(u, n, \xi)}{\delta} \in \operatorname{supp} \psi\right\} .
$$

Consider the velocity-averaged multiplier operator

$$
\overline{M_{\psi} f}(t, x):=\int_{\mathbb{R}} M_{\psi} f(t, x, \xi) \mathrm{d} \xi=\int_{\mathbb{R}} \mathcal{F}_{t x}^{-1} \psi\left(\frac{m(u, n, \xi)}{\delta}\right) \mathcal{F}_{t x} f(u, n, \xi) \mathrm{d} \xi,
$$


then for every $p \in[1,2]$ we have the estimate

$$
\left\|\overline{M_{\psi} f}\right\|_{L_{t, x}^{p}} \leq C \sup _{u, n}|\Omega(u, n ; \delta)|^{1 / p^{\prime}}\|f\|_{L_{t, x, \xi}^{p}} .
$$

Moreover, for every pair $\left(\epsilon, q_{\epsilon}\right)$ satisfying

$$
\frac{N}{q_{\epsilon}^{\prime}}<\epsilon<1<q_{\epsilon}<\frac{N}{N-\epsilon},
$$

where $q_{\epsilon}^{\prime}$ is the conjugate exponent to $q_{\epsilon}$, it holds true that

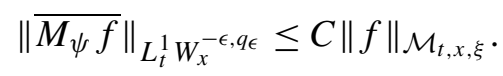

\section{REFERENCES}

[1] Ammar, K. and Wittbold, P. (2003). Existence of renormalized solutions of degenerate elliptic-parabolic problems. Proc. Roy. Soc. Edinburgh Sect. A 133 477-496. MR1983682

[2] Bahouri, H., Chemin, J.-Y. and Danchin, R. (2011). Fourier Analysis and Nonlinear Partial Differential Equations. Springer, Berlin.

[3] Barbu, V., Da Prato, G. and RöcKner, M. (2008). Existence and uniqueness of nonnegative solutions to the stochastic porous media equation. Indiana Univ. Math. J. 57 187-211. MR2400255

[4] Bauzet, C., Vallet, G. and Wittbold, P. (2012). The Cauchy problem for conservation laws with a multiplicative stochastic perturbation. J. Hyperbolic Differ. Equ. 9 661-709. MR3021756

[5] Bauzet, C., Vallet, G. and Wittbold, P. (2015). A degenerate parabolic-hyperbolic Cauchy problem with a stochastic force. J. Hyperbolic Differ. Equ. 12 501-533.

[6] Berthelin, F. and Vovelle, J. (2010). A Bhatnagar-Gross-Krook approximation to scalar conservation laws with discontinuous flux. Proc. Roy. Soc. Edinburgh Sect. A $140953-$ 972. MR2726116

[7] Bouchut, F. and Desvillettes, L. (1999). Averaging lemmas without time Fourier transform and application to discretized kinetic equations. Proc. Roy. Soc. Edinburgh Sect. A 129 19-36.

[8] Carrillo, J. (1999). Entropy solutions for nonlinear degenerate problems. Arch. Ration. Mech. Anal. 147 269-361. MR1709116

[9] CARrillo, J. and Wittbold, P. (1999). Uniqueness of renormalized solutions of degenerate elliptic-parabolic problems. J. Differential Equations 156 93-121.

[10] Chen, C. Q., Ding, Q. and KARLSEN, K. H. (2012). On nonlinear stochastic balance laws. Arch. Ration. Mech. Anal. 204 707-743.

[11] Chen, G.-Q. and Perthame, B. (2003). Well-posedness for non-isotropic degenerate parabolic-hyperbolic equations. Ann. Inst. H. Poincaré Anal. Non Linéaire 20 645-668. MR1981403

[12] CRandall, M. G. (1972). The semigroup approach to first order quasilinear equations in several space variables. Israel J. Math. 12 108-132.

[13] Crandall, M. G. and Liggett, T. M. (1971). Generation of semi-groups of nonlinear transformations on general Banach spaces. Amer. J. Math. 93 265-298.

[14] Da Prato, G., Röckner, M., RozovskiI, B. L. and WANG, F.-Y. (2006). Strong solutions of stochastic generalized porous media equations: Existence, uniqueness, and ergodicity. Comm. Partial Differential Equations 31 277-291. MR2209754 
[15] Da Prato, G. and ZabczyK, J. (1992). Stochastic Equations in Infinite Dimensions. Encyclopedia Math. Appl. 44. Cambridge Univ. Press, Cambridge.

[16] Debussche, A., Hofmanová, M. and Vovelle, J. (2016). Degenerate parabolic stochastic partial differential equations: Quasilinear case. Ann. Probab. 44 1916-1955. DOI:10.1214/15-AOP1013.

[17] Debussche, A. and Vovelle, J. (2015). Invariant measure of scalar first-order conservation laws with stochastic forcing. Probab. Theory Related Fields 163 575-611.

[18] Debussche, A. and Vovelle, J. Scalar conservation laws with stochastic forcing, revised version. Available at http://math.univ-lyon1.fr/vovelle/DebusscheVovelleRevised.pdf.

[19] Debussche, A. and Vovelle, J. (2010). Scalar conservation laws with stochastic forcing. J. Funct. Anal. 259 1014-1042. MR2652180

[20] Deya, A., Gubinelli, M., Hofmanová, M. and Tindel, S. General a priori estimates for rough PDEs with application to rough conservation laws. Available at arXiv:1604.00437.

[21] DiPerna, R. J. and Lions, P. L. (1989). On the Cauchy problem for Boltzmann equations: Global existence and weak stability. Ann. of Math. 130 321-366.

[22] DiPerna, R. J., Lions, P. L. and MeYer, Y. (1991). $L^{p}$ regularity of velocity averages. Ann. Inst. H. Poincaré Anal. Non Linéaire 8 271-287.

[23] Es-SARHIR, A. and VON RENESSE, M.-K. (2012). Ergodicity of stochastic curve shortening flow in the plane. SIAM J. Math. Anal. 44 224-244.

[24] Feng, J. and NuAlart, D. (2008). Stochastic scalar conservation laws. J. Funct. Anal. 255 313-373.

[25] FRIZ, P. K. and Gess, B. (2016). Stochastic scalar conservation laws driven by rough paths. Ann. Inst. H. Poincaré Anal. Non Linéaire 33 933-963. MR3519527

[26] Gess, B. and Souganidis, P. E. (2016). Long-time behavior, invariant measures, and regularizing effects for stochastic scalar conservation laws. Comm. Pure Appl. Math. 701562 1597. MR3666564

[27] Gess, B. and Souganidis, P. E. (2017). Stochastic non-isotropic degenerate parabolichyperbolic equations. Stochastic Process. Appl. 127 2961-3004. MR3682120

[28] Gess, B. and SougAnidis, P. E. (2015). Scalar conservation laws with multiple rough fluxes. Commun. Math. Sci. 13 1569-1597. MR3351442

[29] GeSs, B. and Tölle, J. M. (2014). Multi-valued, singular stochastic evolution inclusions. J. Math. Pures Appl. 9 789-827. MR3205643

[30] Gess, B. and Tölle, J. M. (2016). Ergodicity and local limits for stochastic local and nonlocal $p$-Laplace equations. SIAM J. Math. Anal. 48 4094-4125. MR3580815

[31] Grafakos, L. (2008). Classical Fourier Analysis, 2nd ed. Graduate Texts in Mathematics 249. Springer, New York.

[32] Hofmanová, M. (2013). Degenerate parabolic stochastic partial differential equations. Stochastic Process. Appl. 123 4294-4336.

[33] Hofmanové, M. (2015). A Bhatnagar-Gross-Krook approximation to stochastic scalar conservation laws. Ann. Inst. Henri Poincaré Probab. Stat. 51 1500-1528. MR3414456

[34] HofmanovÁ, M. (2016). Scalar conservation laws with rough flux and stochastic forcing. Stoch. Partial Differ. Equ., Anal. Computat. 4 635-690.

[35] Holden, H. and Risebro, N. H. (1997). Conservation laws with a random source. Appl. Math. Optim. 36 229-241.

[36] Imbert, C. and Vovelle, J. (2004). A kinetic formulation for multidimensional scalar conservation laws with boundary conditions and applications. SIAM J. Math. Anal. 36 214232. MR2083859

[37] Jabin, P. E. and Perthame, B. (2002). Regularity in kinetic formulations via averaging lemmas. ESAIM Control Optim. Calc. Var. 8 761-774 (electronic).

[38] KIM, J. U. (2003). On a stochastic scalar conservation law. Indiana Univ. Math. J. 52 227-256. MR1970028 
[39] Komorowski, T., Peszat, S. and Szarek, T. (2010). On ergodicity of some Markov processes. Ann. Probab. 38 1401-1443. MR2663632

[40] KRUŽKOV, S. N. (1970). First order quasilinear equations with several independent variables. Mat. Sb. 81 228-255.

[41] Lions, P. L., Perthame, B. and Souganidis, P. E. (2013). Scalar conservation laws with rough (stochastic) fluxes. Stoch. Partial Differ. Equ., Anal. Computat. 1 664-686.

[42] Lions, P. L., Perthame, B. and Tadmor, E. (1991). Formulation cinétique des lois de conservation scalaires multidimensionnelles. C. R. Math. Acad. Sci. Paris 97-102. Série I.

[43] Lions, P.-L., Perthame, B. and Tadmor, E. (1994). A kinetic formulation of multidimensional scalar conservation laws and related equations. J. Amer. Math. Soc. 7 169-191. MR1201239

[44] Lions, P.-L., Perthame, B. and Souganidis, P. E. (2014). Scalar conservation laws with rough (stochastic) fluxes: The spatially dependent case. Stoch. Partial Differ. Equ., Anal. Computat. 2 517-538. MR3274890

[45] Perthame, B. (2002). Kinetic Formulation of Conservation Laws. Oxford Lecture Ser. Math. Appl. 21. Oxford Univ. Press, Oxford.

[46] Perthame, B. and Tadmor, E. (1991). A kinetic equation with kinetic entropy functions for scalar conservation laws. Comm. Math. Phys. 136 501-517. MR1099693

[47] Prévôt, C. and Röckner, M. (2007). A Concise Course on Stochastic Partial Differential Equations. Lecture Notes in Math. 1905. Springer, Berlin.

[48] REN, J., RÖCKNER, M. and WANG, F.-Y. (2007). Stochastic generalized porous media and fast diffusion equations. J. Differential Equations 238 118-152.

[49] RÖCKNER, M. and WANG, F.-Y. (2008). Non-monotone stochastic generalized porous media equations. J. Differential Equations 245 3898-3935.

[50] SAussereau, B. and StoicA, I. L. (2012). Scalar conservation laws with fractional stochastic forcing: Existence, uniqueness and invariant measure. Stochastic Process. Appl. 122 $1456-1486$.

[51] TADMOR, E. and TAO, T. (2007). Velocity averaging, kinetic formulations, and regularizing effects in quasi-linear PDEs. Comm. Pure Appl. Math. 60 1488-1521. MR2342955

[52] Triebel, H. (1978). Interpolation Theory, Function Spaces, Differential Operators. NorthHolland Mathematical Library 18. North-Holland, Amsterdam. MR0503903

[53] Vallet, G. and Wittbold, P. (2009). On a stochastic first-order hyperbolic equation in a bounded domain. Infin. Dimens. Anal. Quantum Probab. Relat. Top. 12 613-651. MR2590159

[54] VÁzquez, J. L. (2007). The Porous Medium Equation. Oxford Mathematical Monographs. Oxford Univ. Press, Oxford.

\author{
Max Planck Institute for Mathematics in the Sciences \\ INSELSTRASSE 22 \\ 04103 LEIPZIG \\ GERMANY \\ AND \\ FAKULTÄT FÜR MATHEMATIK \\ UNIVERSITÄT BIELEFELD \\ D-33501 BIELEFELD \\ GERMANY \\ E-MAIL: bgess@mis.mpg.de
}

INSTITUTE OF MATHEMATICS TECHNICAL UNIVERSITY BERLIN STRASSE DES 17. JUNI 136 10623 BERLIN

GERMANY

E-MAIL: hofmanov@math.tu-berlin.de 